JOURNAL OF THE

AMERICAN MATHEMATICAL SOCIETY

Volume 9, Number 4, October 1996

\title{
FINITE DEHN SURGERY ON KNOTS
}

\author{
S. BOYER AND X. ZHANG
}

\section{INTRODUCTION}

Let $K$ be a knot with a closed tubular neighbourhood $N(K)$ in a connected orientable closed 3-manifold $W$, such that the exterior of $K, M=W-\operatorname{int} N(K)$, is irreducible. We consider the problem of which Dehn surgeries on $K$, or equivalently, which Dehn fillings on $M$, can produce 3-manifolds with finite fundamental group. For convenience, a surgery is called a $G$-surgery if the resultant 3-manifold has fundamental group $G$. If $G$ is cyclic or finite, the surgery is also called a cyclic surgery or a finite surgery. Similar terminology will be used for Dehn fillings. The manifold obtained by the Dehn filling on $M$ along $\partial M$ with slope $r$ is denoted by $M(r)$. Let $\Delta\left(r_{1}, r_{2}\right)$ denote the minimal geometric intersection number (the distance) between two slopes $r_{1}$ and $r_{2}$ on $\partial M$.

According to [T1], $M$ belongs to one of the following three mutually exclusive categories:

(I) $M$ is a Seifert fibred space admitting no essential torus.

(II) $M$ is a hyperbolic manifold (i.e. int $M$ admits a complete hyperbolic metric of finite volume).

(III) $M$ contains an essential torus.

It is a remarkable result, the so-called cyclic surgery theorem [CGLS], that if $M$ is not Seifert fibred, then all cyclic surgery slopes of $K$ have mutual distance no larger than 1 , and consequently, there are at most 3 cyclic surgeries on $K$. In this paper, we consider finite Dehn surgery on $K$ and we prove, for instance, that if $M$ is not a manifold of type (I) and is not a union along a torus of the twisted $I$-bundle over the Klein bottle and a cabled space, then there are at most 6 finite and cyclic surgeries on $K$ of maximal mutual distance 5 . Henceforth, we shall use finite/cyclic to mean either finite or (infinite) cyclic.

It turns out to be convenient to consider the three cases mentioned above separately. In case (I) it is well-known that one can completely classify the finite/cyclic surgeries on $K$. Considering the torus knots for instance, one sees that there exist infinitely many knots whose exteriors are of type (I), each of which admit an infinity of distinct finite (cyclic or non-cyclic) surgery slopes. Our contributions deal with the cases (II) and (III). For the former we obtain

Received by the editors October 5, 1993 and, in revised form, May 5, 1995.

1991 Mathematics Subject Classification. Primary 57M25, 57R65.

The first author was partially supported by grants: NSERC OGP 0009446 and FCAR EQ 3518.

The second author was supported by a postdoctoral fellowship from the Centre de Recherches Mathématiques.

(C)1996 American Mathematical Society 
Theorem 1.1. Let $K$ be a knot in a closed connected orientable 3-manifold $W$ such that $M=W-\operatorname{int} N(K)$ is hyperbolic.

(1) There are at most six finite/cyclic surgeries on $K$ and $\Delta\left(r_{1}, r_{2}\right) \leq 5$ for any two finite/cyclic surgery slopes $r_{1}$ and $r_{2}$ of $K$.

(2) If $r_{1}$ is a finite/cyclic surgery slope and $r_{2}$ is a cyclic surgery slope of $K$, then $\Delta\left(r_{1}, r_{2}\right) \leq 2$.

The known realizable maximal number of finite/cyclic surgeries on a knot as in Theorem 1.1, and their maximal mutual distance, is 5 and 3 [W]. See Example 10.5. We note also that Theorem 1.1 (2) is sharp by either Example 10.1 or Example 10.5.

To discuss case (III), we introduce the following notion. A compact connected orientable 3-manifold $M$, with boundary a torus, is called a generalized n-iterated torus knot exterior if $M$ can be decomposed along disjoint essential tori into a union of $n$ cabled spaces and a Seifert fibred space which has a Seifert fibration over the 2-disc with exactly 2 exceptional fibres.

Theorem 1.2. Let $K$ be a knot in a closed connected orientable 3-manifold $W$ such that $M=W-\operatorname{int} N(K)$ is irreducible and contains an essential torus.

(1) If $M$ is not a generalized 1-iterated torus knot exterior, then $\Delta\left(r_{1}, r_{2}\right) \leq 1$ for any two finite/cyclic surgery slopes $r_{1}$ and $r_{2}$ of $K$. In particular, there are at most three finite/cyclic surgeries on $K$.

(2) If $M$ is not a generalized 1- or 2-iterated torus knot exterior, and if $K$ admits an odd order cyclic surgery, then $K$ does not admit any other finite/cyclic surgery.

Finite/cyclic filling on a generalized 1- or 2-iterated torus knot exterior $M$ can be completely described using Gordon's surgery transformation formula [Go]. This is essentially done in [BH, §2], where it is shown that if $M$ is not a union of the twisted $I$-bundle over the Klein bottle and a cabled space, then there are no more than six finite/cyclic fillings of maximal mutual distance 5 (realized on the exterior of $(11,2)$-cable over the $(2,3)$-torus knot in $\left.S^{3}\right)$. In particular, it is proved that an iterated torus knot in $S^{3}$ admitting a non-trivial finite surgery must be a cable over a torus knot, and a complete list of all finite surgeries on cabled knots over torus knots in $S^{3}$ is given in $\S 2$ of that paper.

It is shown in Example 10.6 that Theorem 1.2 (1) is sharp. We also note that as the finite/cyclic fillings on generalized 1- and 2-iterated torus knot exteriors are readily determined, Theorem 1.2 (2) completes the classification of finite/cyclic surgeries on knots in manifolds of odd order cyclic fundamental group whose exteriors contain an essential torus. For some more information concerning finite/cyclic fillings on manifolds of type (III), see the remarks in $\S 8$ where the proof of Theorem 1.2 is given.

A closed orientable 3-manifold $W$ is called small if $W$ does not contain an orientable incompressible surface of positive genus and $\pi_{1}(W)$ has no representation into $P S L_{2}(\mathbf{C})$ with non-cyclic image. We remark that Theorem 1.1 and Theorem 1.2 remain true, with identical proof, if we replace the word "cyclic" by the word "small" in their statements. We also note that should Thurston's geometrization conjecture be shown to hold, then all small manifolds would be either lens spaces, $S^{3}$ or $S^{1} \times S^{2}$.

We now specialize to the most interesting case, surgery on knots in the 3 -sphere $S^{3}$. As is usual, slopes for a knot in $S^{3}$ are parameterized by $\mathbf{Q} \cup\left\{\frac{1}{0}\right\}$ through the use of the standard meridian-longitude coordinates. Note that in $S^{3}$, only the trivial knot admits a $\mathbf{Z}$-surgery [Ga3]. 
Corollary 1.3. Let $K \subset S^{3}$ be a hyperbolic knot.

(1) There are at most six finite surgeries on $K$, and $\Delta\left(r_{1}, r_{2}\right) \leq 5$ for any two finite surgery slopes $r_{1}$ and $r_{2}$ of $K$.

(2) If $r_{1}$ is a finite surgery slope and $r_{2}$ is a cyclic surgery slope of $K$, then $\Delta\left(r_{1}, r_{2}\right) \leq 2$. In particular, if $m / n$ is a finite surgery slope of $K$, then $|n| \leq 2$.

It is shown in $[\mathrm{BH}]$ that the $(-2,3,7)$-pretzel knot admits 4 finite surgeries of maximal mutual distance 2. We prove in Example 10.1 that this knot has no other finite slopes. This example exhibits the known maximal number of finite surgeries on a hyperbolic knot in $S^{3}$.

Corollary 1.4. Let $K \subset S^{3}$ be a satellite knot. If $K$ admits a non-trivial finite surgery, then $K$ is a cabled knot over a torus knot.

It follows from our previous remarks that Corollary 1.4 classifies finite surgeries on satellite knots in $S^{3}$.

In their recent work [BH], Bleiler and Hodgson have obtained, using a completely different approach, the number 24 and the distance 23 for finite/cyclic surgery on a knot as in Theorem 1.1, and the number 8 and the distance 5 for finite/cyclic surgery on a knot as in Theorem 1.2.

It is a classical result that a finite group which is the fundamental group of a 3-manifold must belong to one of the following seven types [Mi]:

C-type. Cyclic groups $\mathbf{Z}_{j}$ for $j \geq 1, \mathbf{Z}_{1}$ being the trivial group.

Even D-type. $D_{4 n} \times \mathbf{Z}_{j}$ with $n \geq 2$ even, $j \geq 1$ and $(n, j)=1$, where $D_{4 n}=$ $\left\{x, y ; x^{2}=(x y)^{2}=y^{n}\right\}$.

Odd D-type. $D\left(2^{k}, 2 l+1\right) \times \mathbf{Z}_{j}$ for $k \geq 2, j \geq 1, l \geq 1,(2(2 l+1), j)=1$, where $D\left(2^{k}, 2 l+1\right)=\left\{x, y ; x^{2^{k}}=1, y^{2 l+1}=1, x y x^{-1}=y^{-1}\right\}$. Note that $D\left(2^{2}, 2 l+1\right)$ is the binary dihedral group $D_{4(2 l+1)}=\left\{x, y ; x^{2}=(x y)^{2}=y^{2 l+1}\right\}$.

T-type. $T\left(8,3^{k}\right) \times \mathbf{Z}_{j}$ for $k \geq 1, j \geq 1,(6, j)=1$, where $T\left(8,3^{k}\right)=\left\{x, y, z ; x^{2}=\right.$ $\left.(x y)^{2}=y^{2}, z^{3^{k}}=1, z x z^{-1}=y, z y z^{-1}=x y\right\}$. Note that $T(8,3)$ is the binary tetrahedral group $T_{24}=\left\{x, y ; x^{2}=(x y)^{3}=y^{3}, x^{4}=1\right\}$.

O-type. $O_{48} \times \mathbf{Z}_{j}$ for $j \geq 1,(6, j)=1$, where $O_{48}=\left\{x, y ; x^{2}=(x y)^{3}=y^{4}\right.$, $\left.x^{4}=1\right\}$ is the binary octahedral group.

I-type. $I_{120} \times \mathbf{Z}_{j}$ for $j \geq 1,(30, j)=1$, where $I_{120}=\left\{x, y ; x^{2}=(x y)^{3}=y^{5}\right.$, $\left.x^{4}=1\right\}$ is the binary icosahedral group.

Q-type. $Q(8 n, k, l) \times \mathbf{Z}_{j}$, where $Q(8 n, k, l)=\left\{x, y, z ; x^{2}=(x y)^{2}=y^{2 n}, z^{k l}=\right.$ $\left.1, x z x^{-1}=z^{r}, y z y^{-1}=z^{-1}\right\}, n, k, l, j$ are relatively prime odd positive integers, $r \equiv-1(\bmod k)$ and $r \equiv 1(\bmod l)$.

The groups occurring in the first six types are precisely the finite subgroups of the special orthogonal group $S O(4)$ which act freely on the 3 -sphere $S^{3}$, and thus, they are fundamental groups of spherical space forms ([Hf]). We note that each such space form is a Seifert fibred manifold, and further, that a Seifert fibred manifold with finite non-cyclic fundamental group has a Seifert fibration over $S^{2}$ with exactly 3 exceptional fibres whose indices $\alpha_{1}, \alpha_{2}$ and $\alpha_{3}$ form a platonic triple, i.e. satisfying $1 / \alpha_{1}+1 / \alpha_{2}+1 / \alpha_{3}>1$ (see [J, IV.11]).

The following results give more precise information on finite/cyclic surgeries of a given type. 
Theorem 1.5. Let $K$ be a knot in a closed connected orientable 3-manifold $W$ such that $M=W-\operatorname{int} N(K)$ is hyperbolic.

(1) If $r_{1}$ is a D-type or $Q$-type slope of $K$, and $r_{2}$ is a cyclic surgery slope of $K$, then $\Delta\left(r_{1}, r_{2}\right)=1$.

(2) Suppose that $H_{1}(M)=\mathbf{Z} \oplus \mathbf{Z}_{p}$ with $p$ odd.

(i) There is at most one $D$-type finite surgery on $K$.

(ii) If there is a D-type surgery on $K$, then there is no even order cyclic surgery on $K$.

(iii) If there is a D-type surgery on $K$, then there are at most two cyclic surgeries on $K$.

(3) Suppose that $H_{1}(M)=\mathbf{Z} \oplus \mathbf{Z}_{p}$ with $p$ odd.

(i) If there is an $O$-type surgery on $K$, then there is no even order cyclic surgery on $K$.

(ii) If $r_{1}$ is an O-type slope and $r_{2}$ is a cyclic surgery slope, then $\Delta\left(r_{1}, r_{2}\right)=1$.

(iii) There are at most two O-type surgeries on $K$ and if two, their distance is 4.

(iv) If $r_{1}$ is an O-type slope and $r_{2}$ a D-type slope, then $\Delta\left(r_{1}, r_{2}\right)=2$.

(4) Suppose that $H_{1}(M)=\mathbf{Z} \oplus \mathbf{Z}_{p}$ with $p$ relatively prime to 3 . Then there are at most two $T$-type surgeries on $K$, and if two, their distance is 3 .

For knots in $S^{3}$, Theorem 1.5 yields the following corollary. Note first that if $\alpha$ is a finite slope for such a knot $K$, say $\alpha=m / n$, then (i) $m$ is divisible by 4 if $\alpha$ is of $D$-type, (ii) $m$ is odd and divisible by 3 if $\alpha$ is of $T$-type, and (iii) $m$ is divisible by 2 , but not by 3 or 4 , if $\alpha$ is of $O$-type (see Lemma 5.1).

Corollary 1.6. Let $K \subset S^{3}$ be a hyperbolic knot.

(1) (i) Any D-type slope of $K$ must be an integral slope.

(ii) There is at most one $D$-type finite surgery on $K$.

(iii) If there is a D-type surgery on $K$, then there is no even order cyclic surgery on $K$.

(iv) If there is a D-type surgery $\alpha$ on $K$, then there is at most one non-trivial cyclic surgery on $K$, and if there is one, $\beta$ say, then $\alpha$ and $\beta$ are consecutive integers.

(2) (i) Any O-type slope of $K$ must be an integral slope.

(ii) There are at most two $O$-type slopes on $K$ and if two, their distance is 4 .

(iii) If there is an O-type surgery on $K$, then there is no even order cyclic surgery on $K$.

(iv) If there is an $O$-type surgery $\alpha$ on $K$, then there is at most one non-trivial cyclic surgery on $K$, and if there is one, $\beta$ say, then $\alpha$ and $\beta$ are consecutive integers.

(v) If there is an O-type surgery slope and a D-type surgery slope on $K$, then they are consecutive even integers.

(3) There are at most two $T$-type slopes on $K$, and if two, one is integral, the other has denominator 2 , and their distance is 3 .

Corollary 1.6 may be complemented by various examples of hyperbolic knots in $S^{3}$ which admit $O$-type or $I$-type or $D$-type surgery ([BH]). In $\S 10$, we give a few more examples. Notably Example 10.2 provides a hyperbolic knot in $S^{3}$ which admits a $D$-type surgery and an odd order non-trivial cyclic surgery, showing that Theorem 1.5 (2) is sharp, and Example 10.4 provides a hyperbolic knot in $S^{3}$ which 
admits a $T$-type surgery and a non-trivial cyclic surgery. Theorem 1.5 (4) is also sharp by Example 10.5.

Corollary 1.7. If $K \subset S^{3}$ is a non-trivial amphicheiral knot, then only $\pm 1, \pm 1 / 2$ and \pm 2 can possibly be non-trivial finite surgery slopes of $K$. In particular, only $I_{120}$ and $O_{48}$ may occur as fundamental group.

Proof. Since non-trivial torus knots and cabled knots in $S^{3}$ are not amphicheiral knots, we may assume, by Corollary 1.4, that $K$ is a hyperbolic knot. Let $M=$ $S^{3}-\operatorname{int} N(K)$. Note that as $K$ is amphicheiral, $M(r)$ is homeomorphic to $M(-r)$ for all slopes $r$. Suppose that $m / n$ is a non-trivial finite surgery slope of $K$. Then $-m / n$ is also a finite surgery slope, giving the same finite fundamental group as $m / n$ does. Now $\Delta(m / n,-m / n)=2|m n|$ and thus $|m n| \leq 2$ by Theorem 1.1 (1). Thus only $\pm 1, \pm 1 / 2$ and \pm 2 can possibly be non-trivial finite surgery slopes of $K$. It follows that the first homology group of a non-trivial, finite surgery on $K$ is either 0 or $\mathbf{Z}_{2}$. Consideration of the abelianizations of the seven types of groups shows that the fundamental group of such a surgery is either the trivial group, $\mathbf{Z}_{2}$, $I_{120}$ or $O_{48}$. It follows from the cyclic surgery theorem of [CGLS] that the first two groups do not occur from non-trivial surgery on an amphicheiral knot. Thus the proof of the corollary is complete.

A knot $K$ in $S^{3}$ is said to satisfy property $I$ if no surgery on $K$ can yield a manifold with fundamental group the binary isosahedral group $I_{120}$, and it is conjectured that every knot in $S^{3}$, except the trefoil knot, satisfies property I [Z]. Property I has been proved for several classes of knots in $S^{3}$, including satellite knots and nontrefoil torus knots [Z]. Note that an $I_{120}$ surgery slope of a knot $K$ in $S^{3}$ is of the form $1 / n$. Hence, applying Theorem 1.1 (2), we have

Corollary 1.8. If a non-trefoil knot $K$ in $S^{3}$ does not satisfy property I, then $K$ is a hyperbolic knot and the $I_{120}$-surgery slopes that $K$ admits can only be \pm 1 and $\pm 1 / 2$.

Based on the results obtained in this paper and known examples, we raise the following problem.

Finite/cyclic surgery problem. (I) Let $K$ be a knot in a connected closed orientable 3-manifold $W$ such that $M=W-i n t N(K)$ is a hyperbolic manifold. Show that there are at most five finite/cyclic surgeries on $K$ and the distance between any two finite/cyclic surgery slopes is at most 3.

(II) Let $K \subset S^{3}$ be a hyperbolic knot. Show that

(1) there are at most four finite surgeries on $K$;

(2) the non-trivial finite surgery slopes on $K$ form a set of consecutive integers;

(3) the distance between any two finite surgery slopes of $K$ is at most 2;

(4) there is at most one finite surgery on $K$ with an even integral slope.

This paper provides some evidence supporting a positive solution to this problem. For instance, if the minimal norm (see $\S 2)$ of a non-trivial element of $H_{1}(\partial M ; \mathbf{Z})$ is greater than 24 (16 for knots whose exteriors have no 2-torsion in their homology), then the methods of this paper show that for a knot $K$ as in Theorem 1.1, there are at most four finite surgeries on $K$ of maximal mutual distance no more than 2. These methods may also be used to obtain results concerning finite surgeries on certain families of knots. For instance D. Tanguay has used this approach to 
show that the fundamental group of a manifold resulting from surgery along a non-meridinal slope of a hyperbolic 2-bridge knot is infinite ([Ta]).

Our approach follows that developed in [CGLS] for analyzing cyclic surgery slopes. Let $M$ be a compact, connected, orientable, irreducible 3-manifold such that $\partial M$ is a torus. By an essential surface in $M$, we mean a properly embedded, orientable, incompressible, $\partial$-incompressible, non- $\partial$-parallel surface $F$ in $M$. A slope $r \subset \partial M$ is called a boundary slope if there is an essential surface $F$ in $M$ such that $\partial F$ is a non-empty set of parallel simple closed curves on $\partial M$ of slope $r$; if further, $F$ is not a fibre in any fibration of $M$ over the circle, $r$ is called a strict boundary slope. The proof of the cyclic surgery theorem consists of essentially two parts, accomplished in Chapter 1 and Chapter 2 of [CGLS] respectively. Part one deals with the case that $M$ is a hyperbolic manifold and cyclic surgery slopes are not strict boundary slopes; part two deals with the case that either $M$ is hyperbolic and one of cyclic surgery slopes is a strict boundary slope, or $M$ contains an essential torus. For the finite surgery problem, the results of Chapter 2 of [CGLS] apply directly. Also, most of the results and machinery of Chapter 1 of [CGLS] work and can be adapted to our situation. This is the case in Theorem 2.1 where we generalize [CGLS, Corollary 1.1.4] to obtain a multiplicative bound on the Culler-Shalen norm (see $\S 2$ for notation and terminology) of a slope which lifts to a cyclic slope in some finite, regular cover of $M$. On the other hand, in our goal to develop sharp bounds for the finite surgery problem, a major obstacle occurs when estimating the order of a zero of the function $f_{\alpha}$ associated to a finite surgery class $\alpha \in H_{1}(M ; \mathbf{Z})$ at an ordinary point $x$ of the smooth model of the canonical curve in the character variety of $\pi_{1}(M)$. The following theorem, which is basically the same as Theorem 2.2, resolves this obstacle and represents one of the main contributions of the paper.

Theorem 1.9. Suppose that $\alpha \in L$ is a finite surgery class and that $Z_{x}\left(f_{\alpha}\right)>$ $Z_{x}\left(f_{\beta}\right)$ for some non-zero element $\beta \in L$, and some ordinary point $x$ of the smooth model of the canonical curve in the character variety of $\pi_{1}(M)$. Then

(1) $x$ corresponds to a smooth point of the canonical curve;

(2) $Z_{x}\left(f_{\alpha}\right)=2$ and $Z_{x}\left(f_{\beta}\right)=0$.

Theorem 1.9 is the key to proving that the norm of a finite surgery class is essentially a count of the number of characters of irreducible representations of $\pi_{1}(M(\alpha))$ to $S L_{2}(\mathbf{C})$.

We begin in $\S 2$ by reviewing some necessary background material from [CGLS, Chapter 1]. Theorem 2.1 is then proven in $\S 3$, while Theorem 2.2 (and hence Theorem 1.9) is proven in $\S 4$. Next, based on Theorems 2.1 and 2.2, we obtain an estimate (Theorem 2.3) of the norm $\|\alpha\|$ of a finite surgery class $\alpha$ which is not a strict boundary class. This is content of $\S 5$. We note that as an immediate consequence of Theorem 2.3 and [CGLS, Theorem 2.0.3], an approximation may be obtained of the number of distinct finite/cyclic surgery slopes and their maximal mutual distance. To obtain a finer approximation (Theorem 1.1), a detailed analysis is carried out in $\S 6$ and $\S 7$ on how many finite/cyclic non-boundary classes that a $\|\cdot\|$-disc of a certain radius can possibly contain, and how large the distance between these classes can possibly be.

Theorem 1.2 is proven in $\S 8$ by applying Theorem 1.1 and results from [Ga1], [Ga2], [Sch], [Go] and [CGLS] to the torus decomposition of $M$. The proof of Theorem 1.5 is given in $\S 9$, based mainly on Theorem 1.1 and Theorem 2.3. Finally, $\S 10$ of the paper consists of the examples mentioned above. 
The authors would like to thank Marc Troyanov for several valuable conversations concerning the material of this paper.

\section{THE NORM $\|\cdot\|$}

In this section, we briefly review some of the machinery developed in [CGLS] for analyzing cyclic surgeries, and we describe how one can proceed further to obtain the finite/cyclic surgery theorem (Theorem 1.1).

Throughout this section, $M$ denotes a connected, orientable, compact, irreducible 3-manifold such that $\partial M$ is a torus, and $M$ is hyperbolic. Some conventions are in order. Excepting $§ 3$, the base point of fundamental group will be suppressed throughout, $\pi_{1}(\partial M)$ will be identified with its image under the natural inclusion $\pi_{1}(\partial M) \rightarrow \pi_{1}(M)$ (defined up to conjugation). The group $H_{1}(\partial M ; \mathbf{Z})$ will be denoted by $L$, and will also be regarded as a lattice in the 2-dimensional real vector space $V=H_{1}(\partial M ; \mathbf{R})$. Elements in $L$ will be written additively or multiplicatively depending on which happens to be the more convenient. The lattice $L$ will often be identified with $\pi_{1}(\partial M)$ using the Hurewicz isomorphism. Recall that each slope $r$ corresponds to a pair of primitive elements, $\pm \delta$, of $L$ and so $M(r)$ will also be denoted by $M(\delta)$. If $r$ is a certain "type" of slope, then each of the elements of the corresponding pair $\pm \delta \in L$ will be said to be that "type" of class, e.g. if $r$ is a strict boundary slope then both $\delta$ and $-\delta$ will be called strict boundary classes. If $\delta_{1}$ and $\delta_{2}$ are elements in $L$, then $\Delta\left(\delta_{1}, \delta_{2}\right)$ will denote the absolute value of their intersection number.

By [CGLS, Chapter 1], there exists a norm $\|\cdot\|$ on $V$ satisfying the following three properties.

(1) $\|\cdot\|$ is positive integer valued for each non-trivial element $\delta \in L$.

(2) Let $s=\min \{\|\delta\| ; \delta \in L, \delta \neq 0\}$ and let $B$ be the disc of radius $s$ in $V$. Then $B$ is a compact, convex, finite-sided, balanced polygon, whose vertices are rational multiples of strict boundary classes in $L$.

(3) If $\alpha \in L$ is a primitive element which is not a strict boundary class and if $M(\alpha)$ has cyclic fundamental group, then $\|\alpha\|=s$.

Consequently, it can be proved ([CGLS, page 244]) that if $\alpha_{1}$ and $\alpha_{2}$ are two elements in $L$, neither of which is a strict boundary class, and if both $\pi_{1}\left(M\left(\alpha_{1}\right)\right)$ and $\pi_{1}\left(M\left(\alpha_{2}\right)\right)$ are cyclic, then $\Delta\left(\alpha_{1}, \alpha_{2}\right) \leq 1$.

The norm is constructed as follows (see [CGLS, Chapter 1] for more details). The set $R=H o m\left(\pi_{1}(M), S L_{2}(\mathbf{C})\right)$ of representations of $\pi_{1}(M)$ in $S L_{2}(\mathbf{C})$ forms a complex affine algebraic set in a natural way. Denote by $X$ the associated set of characters of the representations in $R$, and by $t: R \rightarrow X$ the natural map which sends a representation $\rho \in R$ to its character $\chi_{\rho}$. It so happens that $X$ also admits the structure of a complex affine algebraic set in such a way that the map $t$ becomes a regular map. For each $\gamma \in \pi_{1}(M)$, the function $I_{\gamma}: X \rightarrow \mathbf{C}$ defined by $I_{\gamma}\left(\chi_{\rho}\right)=$ $\chi_{\rho}(\gamma)=\operatorname{trace}(\rho(\gamma))$ is a regular function. Let $R_{0}$ be an irreducible component of $R$ containing a discrete, faithful representation of $\pi_{1}(M)$. Then $X_{0}=t\left(R_{0}\right)$ is an irreducible affine variety of dimension 1 . For any element $\delta \in L=H_{1}(\partial M ; \mathbf{Z})$, considered as an element in $\pi_{1}(\partial M) \subset \pi_{1}(M)$, define the function $f_{\delta}: X_{0} \rightarrow \mathbf{C}$ by $f_{\delta}=I_{\delta}^{2}-4$. The function $I_{\delta}: X_{0} \rightarrow \mathbf{C}$ (and thus $f_{\delta}$ ) is non-constant on $X_{0}$ for each non-zero element $\delta \in L$ ([CGLS, Prop.1.1.1]). Let $\tilde{X}_{0}$ be the smooth projective variety which is birationally equivalent to $X_{0}$. The birational equivalence induces an isomorphism between the function fields $\mathbf{C}\left(X_{0}\right)$ and $\mathbf{C}\left(\tilde{X}_{0}\right)$ and thus any rational 
function $f$ on $X_{0}$ pulls back to a rational function on $\tilde{X}_{0}$ which will also be denoted $f$. Now, the birational equivalence from $\tilde{X}_{0}$ to $X_{0}$ is regular at all but a finite number of points of $\tilde{X}_{0}$; the points where it is defined are called ordinary points, while those that remain are called ideal points. Let $Z_{x}(f)$ (respectively $\Pi_{x}(f)$ ) denote the order of zero (respectively the order of pole) of $f \in \mathbf{C}\left(\tilde{X}_{0}\right)$ at $x \in \tilde{X}_{0}$, setting $Z_{x}(f)=0$ (respectively $\Pi_{x}(f)=0$ ) if $f(x) \neq 0$ (respectively if $f(x) \neq \infty$ ). Then for $\delta \in L$,

$$
\|\delta\|=\sum_{x \in \tilde{X}_{0}} \Pi_{x}\left(f_{\delta}\right)=\sum_{x \in \tilde{X}_{0}} Z_{x}\left(f_{\delta}\right)
$$

That is, $\|\delta\|$ is equal to the degree of $f_{\delta}$.

Property (3) of the norm, listed above, is obtained by showing that for any $x \in \tilde{X}_{0}, Z_{x}\left(f_{\alpha}\right)$ is always the minimal among all elements in $\left\{Z_{x}\left(f_{\delta}\right) ; \delta \in L, \delta \neq 0\right\}$.

Now, consider a finite/cyclic class $\alpha \in L$. If one could obtain a finite bound on the norm of any such $\alpha$, then a distance estimation would follow. Our crucial results concerning these bounds are contained in Theorems 2.1, 2.2, and 2.3 below. To help orient the reader we shall first give a brief description of these results and how they relate to the proof of Theorem 1.1.

Let $\alpha \in L$ be a finite surgery class. It turns out that we need only consider the case where $\alpha$ is not a strict boundary slope, so we shall assume this for the moment. As we mentioned above, if $\alpha$ is a cyclic such class, then its norm realizes the minimal norm amongst all non-zero lattice elements. This is not the case when $\alpha$ is not a cyclic class, but we observe in $\S 5$ that if the fundamental group of a 3-manifold is finite, then it contains a relatively large cyclic normal subgroup. In Theorem 2.1 we show that by passing to the associated cover, we can use the method of [CGLS, Chapter 1] to see that $\|\alpha\|$ is minimal amongst the non-zero elements of a sublattice a $L$ whose index is no greater than 5 . It follows that $\|\alpha\| \leq 5 s$ and so weak estimates for the distance between two such finite classes may now be obtained. To obtain sharp estimates, a bound for the difference $\|\alpha\|-s$ must also be found. The key to accomplishing this task rests on the two results contained in Theorem 2.2. They give us a precise understanding of the jumps in the values of $Z_{x}\left(f_{\alpha}\right)$ where $x$ is an ordinary point in $\tilde{X}_{0}$. More precisely we show that if $Z_{x}\left(f_{\alpha}\right)>Z_{x}\left(f_{\beta}\right)$ for some non-zero element $\beta \in L$, and some $x \in X_{0}^{\nu}$, then $Z_{x}\left(f_{\alpha}\right)=2$, and $Z_{x}\left(f_{\beta}\right)=0$. Furthermore $\nu(x)$ is a smooth point of $X_{0}$. It will follow that $\|\alpha\|-s$ is bounded by twice the number of characters of irreducible representations of $\pi_{1}(M(\alpha))$ to $S L_{2}(\mathbf{C})$. The count of these characters is applied with Theorems 2.1 and 2.2 to yield the inequalities of Theorem 2.3. This latter result is then key in the proof of Theorem 1.1. We now give a more complete description of these results.

Consider a finite surgery class $\alpha \in L$ such that there is a degree $d<\infty$ regular branched cover $\widetilde{M(\alpha)} \rightarrow M(\alpha)$, with branched set in $M(\alpha)$ the core of the sewn solid torus, and with $\pi_{1}(\widetilde{M(\alpha)})$ a cyclic group. Let $k$ be the ramification index of the branched set ( $k=1$ is allowed). Let $p: \tilde{M} \rightarrow M$ be the restriction of this cover to $M$, and write

$$
\partial \tilde{M}=\tilde{T}_{1} \cup \cdots \cup \tilde{T}_{n},
$$

where each $\tilde{T}_{i}$ is a torus. Assume that the base points for $M$ and $\tilde{M}$ lie in $\partial M$ and $\tilde{T}_{1}$ respectively, and that they correspond under $p$. Let $\widetilde{L}=H_{1}\left(\tilde{T}_{1} ; \mathbf{Z}\right)$, which may 
be identified with a subgroup of $\pi_{1}(\tilde{M})$. The following theorem generalizes [CGLS, Corollary 1.1.4].

Theorem 2.1. Suppose that $\alpha$ is not a strict boundary class.

(1) If $k=1$, then $\|\alpha\|=\min \left\{\left\|p_{\#}(\tilde{\beta})\right\| ; \tilde{\beta} \in \widetilde{L}-\{0\}\right\} \leq(d / n) s$.

(2) If $k>1$, then either $k\|\alpha\|=\min \left\{\left\|p_{\#}(\tilde{\beta})\right\| ; \tilde{\beta} \in \widetilde{L}-\{0\}\right\} \leq(d / n) s$, or $M$ contains a closed essential surface of genus greater than 1 .

To explain Theorem 2.2, recall from [CGLS, page 255] that there is a commutative diagram

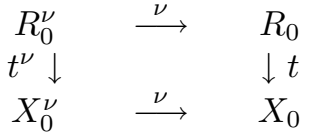

where $R_{0}^{\nu} \stackrel{\nu}{\longrightarrow} R_{0}$ and $X_{0}^{\nu} \stackrel{\nu}{\longrightarrow} X_{0}$ are normalizations ([Sh, Chapter II, §5]). Each of the maps in this diagram is surjective, and the two horizontal arrows are finiteto-one birational equivalences. Further, $X_{0}^{\nu}$ is a non-singular affine curve. As in [CGLS, 1.5], we may identify the ordinary points of $\tilde{X}_{0}$ with $X_{0}^{\nu}$.

Theorem 2.2. Suppose that $\alpha \in L$ is a finite surgery class and that $Z_{x}\left(f_{\alpha}\right)>$ $Z_{x}\left(f_{\beta}\right)$ for some non-zero element $\beta \in L$, and some $x \in X_{0}^{\nu}$. Then

(1) $\nu(x)$ is a smooth point of $X_{0}$;

(2) $Z_{x}\left(f_{\alpha}\right)=2$, and $Z_{x}\left(f_{\delta}\right)>0$ implies $Z_{x}\left(f_{\delta}\right) \geq 2$ for any $\delta \in L$ (thus $Z_{x}\left(f_{\beta}\right)=$ $0)$.

Note that Theorem 2.2 subsumes Theorem 1.9 of the introduction.

Recall that cyclic classes that are not strict boundary classes realize the minimal norm of the non-trivial elements in $L$. While this is not in general true for finite classes, the next result, based on Theorems 2.1 and 2.2, shows that it almost is.

Theorem 2.3. Let $s=\min \left\{\|\delta\| ; \delta \in L=H_{1}(\partial M ; \mathbf{Z}), \delta \neq 0\right\}$. Suppose that $\alpha \in L$ is a finite surgery class and is not a strict boundary class.

(1) If $\alpha$ is a $C$-type class, then $\|\alpha\|=s$.

(2) If $\alpha$ is a D-type or a $Q$-type class, then (i) $\|\alpha\| \leq 2 s$ and (ii) there is a sublattice $\tilde{L} \subset L$ of index 2 such that $\alpha \in \tilde{L}$ and $\|\alpha\| \leq\|\beta\|$ for any non-trivial element $\beta \in \tilde{L}$.

(3) If $\alpha$ is a T-type class, then (i) $\|\alpha\| \leq s+4$ and (ii) there is a sublattice $\tilde{L} \subset L$ of index $q=2$ or 3 such that $\alpha \in \tilde{L}$ and $\|\alpha\| \leq\|\beta\|$ for any non-trivial element $\beta \in \tilde{L}$.

(4) If $\alpha$ is an I-type class, then (i) $\|\alpha\| \leq s+8$ and (ii) there is a sublattice $\tilde{L} \subset L$ of index $q=2$ or 3 or 5 such that $\alpha \in \tilde{L}$ and $\|\alpha\| \leq\|\beta\|$ for any non-trivial element $\beta \in \tilde{L}$.

(5) (a) If $\alpha$ is an $O$-type class and if $H_{1}(M ; \mathbf{Z})$ has no non-trivial even torsion, then (i) $\|\alpha\| \leq s+6$ and (ii) if $\|\alpha\|>s+2$, then there is a sublattice $\tilde{L} \subset L$ of index $q=2$ or 3 or 4 such that $\alpha \in \tilde{L}$ and $\|\alpha\| \leq\|\beta\|$ for any non-trivial element $\beta \in \tilde{L}$.

(b) If $\alpha$ is an $O$-type class and if $H_{1}(M ; \mathbf{Z})$ has non-trivial even torsion, then (i) $\|\alpha\| \leq s+12$ and (ii) if $\|\alpha\|>s+4$, then there is a sublattice $\tilde{L} \subset L$ of index $q=2$ or 3 such that $\alpha \in \tilde{L}$ and $\|\alpha\| \leq\|\beta\|$ for any non-trivial element $\beta \in \tilde{L}$. 
Remarks. (1) Theorem 2.3 (1) is one of the main results in [CGLS].

(2) In Theorem 2.3, a consequence of part (ii) of statements (2) through (5) is:

(i) if $\alpha$ is a $D$-type or a $Q$-type class, then $\|\alpha\| \leq\left\|\alpha \beta^{2}\right\|$ for any element $\beta \in L$;

(ii) if $\alpha$ is a $T$-type class, then $\|\alpha\| \leq\left\|\alpha \beta^{q}\right\|$ for any element $\beta \in L$, and if $\alpha \beta^{q}=\delta^{m}$ for some $\delta \in L$ and some integer $m$ relative prime to $q$, then $\|\alpha\| \leq\|\delta\|$, where $q$ is an integer which is either 2 or 3 ;

(iii) if $\alpha$ is an $I$-type class, then $\|\alpha\| \leq\left\|\alpha \beta^{q}\right\|$ for any element $\beta \in L$, and if $\alpha \beta^{q}=\delta^{m}$ for some $\delta \in L$ and some integer $m$ relative prime to $q$, then $\|\alpha\| \leq\|\delta\|$, where $q$ is an integer which is either 2,3 , or 5 ;

(iv) if $\alpha$ is an $O$-type class and if $\|\alpha\|>s+4$, then $\|\alpha\| \leq\left\|\alpha \beta^{q}\right\|$ for any $\beta \in L$, and (v) if $\alpha \beta^{q}=\delta^{m}$ for some $\delta \in L$ and some integer $m$ relative prime to $q$, then $\|\alpha\| \leq\|\delta\|$, where $q$ is an integer which is either 2 or 3 or 4 ; moreover $q \neq 4$ when $\|\alpha\|>s+6$.

Theorems 2.1, 2.2 and 2.3 will be proved in $\S 3, \S 4$ and $\S 5$ respectively.

\section{Proof of Theorem 2.1}

The general idea of the proof is to arrange things so that we may apply the machinery developed in $\$ 1.5$ and $\$ 1.6$ of [CGLS].

Let $x_{0} \in \partial M$ and for each $i=1, \ldots, n$, fix a point $\tilde{x}_{i} \in \tilde{T}_{i} \cap p^{-1}\left(x_{0}\right)$. We set $\pi=\pi_{1}\left(M, x_{0}\right)$ and $\tilde{\pi}=\pi_{1}\left(\tilde{M}, \tilde{x}_{1}\right)$. Then $p_{\#}: \tilde{\pi} \rightarrow \pi$ is an injective homomorphism whose image is an index $d$ normal subgroup of $\pi$. We choose paths $\tilde{\gamma}_{1}, \ldots, \tilde{\gamma}_{n}$ in $\tilde{M}$ such that $\tilde{\gamma}_{i}$ goes from $\tilde{x}_{1}$ to $\tilde{x}_{i}$, and we require $\tilde{\gamma}_{1}$ to be the constant path. Denote by $\gamma_{i}$ the class in $\pi$ corresponding to the loop $p\left(\tilde{\gamma}_{i}\right), 1 \leq i \leq n$.

Recall that $\rho_{0}$ is a discrete and faithful representation of $\pi$ into $S L_{2}(\mathbf{C})$, and that $R_{0}$ is an irreducible component of $R(\pi)$ containing $\rho_{0}$. There is a regular map $p^{\#}: R(\pi) \rightarrow R(\tilde{\pi})$ given by $p^{\#}(\rho)=\rho \circ p_{\#}$, and if we denote by $S_{0}$ the Zariski closure of $p^{\#}\left(R_{0}\right)$ in $R(\tilde{\pi})$, then $S_{0}$ is necessarily irreducible (as $R_{0}$ is), and $p^{\#}\left(R_{0}\right)$ contains a Zariski open subset of $S_{0}$ [Sh, Theorem 6, page 50].

Lemma 3.1. $S_{0}$ is closed under conjugation by elements of $S L_{2}(\mathbf{C})$.

Proof. This is known to be true for $R_{0}$ ([CS, Proposition 1.1.1]) and thus, it is also true for $p^{\#}\left(R_{0}\right)$. Now with respect to the classical topology, $p^{\#}\left(R_{0}\right)$ is dense in $S_{0}$, and further, $S_{0}$ is closed in $R(\tilde{\pi})$. It follows easily that $S_{0}$ is closed under conjugation by elements of $S L_{2}(\mathbf{C})$.

Next we consider the regular map $t: R(\tilde{\pi}) \rightarrow X(\tilde{\pi})$ which associates to a representation $\rho$ its character $\chi_{\rho}$. As above, if we define $Y_{0}$ to be the Zariski closure of $t\left(S_{0}\right)$ in $X(\tilde{\pi})$, then $Y_{0}$ is irreducible. Further, owing to the fact that $S_{0}$ is closed under conjugation, the proof of [CS, Proposition 1.4.4] may be used to deduce that $t\left(S_{0}\right)=Y_{0}$.

Let $p^{*}: X_{0} \rightarrow Y_{0}$ be the regular map given by $p^{*}\left(\chi_{\rho}\right)=\chi_{p^{\#}(\rho)}$. Then we have the following commutative diagram of regular dominating maps, the two vertical maps being surjective:

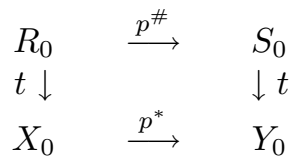

Lemma 3.2. $\operatorname{dim}_{\mathbf{C}}\left(Y_{0}\right)=1$. 
Proof. The map $p^{*}: X_{0} \rightarrow Y_{0}$ is dominating, so that $\operatorname{dim}\left(Y_{0}\right) \leq \operatorname{dim}\left(X_{0}\right)=1$. On the other hand, if $\tilde{\beta} \in \widetilde{L}-\{0\}$, then $p_{\#}(\tilde{\beta}) \in L-\{0\}$, so that $f_{p_{\#}(\tilde{\beta})}$ is nonconstant. But $f_{p_{\#}(\tilde{\beta})}=f_{\tilde{\beta}} \circ p^{*}$ and therefore, $f_{\tilde{\beta}}: Y_{0} \rightarrow \mathbf{C}$ is non-constant. It follows that $\operatorname{dim}_{\mathbf{C}}\left(Y_{0}\right) \geq 1$.

Note that $p^{\#}\left(\rho_{0}\right) \in S_{0}$ is a discrete and faithful representation of $\tilde{\pi}$, and further, that $\tilde{M}$ has finite volume. It follows that $p^{\#}\left(\rho_{0}\right)$ is an irreducible representation and therefore, arguing as in [CS, Corollary 1.5.3], we deduce that $\operatorname{dim}_{\mathbf{C}}\left(S_{0}\right)=4$.

We record the following lemma for later use.

Lemma 3.3. Let $\beta$ be a loop in $\partial M$ based at $x_{0}$, which lifts to loops $\tilde{\beta}_{1}, \ldots, \tilde{\beta}_{n}$ based at $\tilde{x}_{1}, \ldots, \tilde{x}_{n}$. If $[\cdot]$ designates class in a fundamental group, then the regular functions $f_{\left[\tilde{\gamma}_{i} \tilde{\beta}_{i} \tilde{\gamma}_{i}^{-1}\right]}: Y_{0} \rightarrow \mathbf{C}$ are all equal.

Proof. Since $p^{*}\left(X_{0}\right)$ is dense in $Y_{0}$, it suffices to prove that the functions $f_{\left[\tilde{\gamma}_{i} \tilde{\beta}_{i} \tilde{\gamma}_{i}^{-1}\right]^{\circ}}$ p are identical. But one readily verifies that each of these functions equals $f_{[\beta]}$, and so the result follows.

Consider the following commutative diagram of normalizations and projective desingularizations:

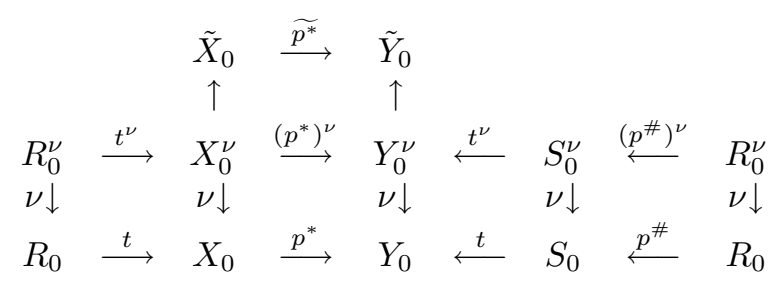

We remark that as $S_{0}$ is closed under conjugation (Lemma 3.1), the method of proof of [CGLS, Proposition 1.5.6] shows that $t^{\nu}: S_{0}^{\nu} \rightarrow Y_{0}^{\nu}$ is surjective.

Lemma 3.4. For each class $\tilde{\beta} \in \tilde{L}, f_{p_{\#}(\tilde{\beta})}=f_{\tilde{\beta}} \widetilde{p^{*}}$. Thus

(1) $\left\|p_{\#}(\tilde{\beta})\right\|=\operatorname{degree}\left(\widetilde{p^{*}}\right) \operatorname{degree}\left(f_{\tilde{\beta}}\right)$;

(2) degree $\left(\widetilde{p^{*}}\right) \neq 0$ and so $\widetilde{p^{*}}$ is onto.

Proof. The identity $f_{p_{\#}(\tilde{\beta})}=f_{\tilde{\beta}}{\widetilde{p^{*}}}$ clearly holds when restricted to $X_{0}$, which proves $f_{p_{\#}(\tilde{\beta})}=f_{\tilde{\beta}} \circ \widetilde{p}^{*}$ on $\tilde{X}_{0}$. If $\tilde{\beta} \neq 0$, then $p_{\#}(\tilde{\beta}) \neq 0$ and thus, $0 \neq$ $\left\|p_{\#}(\tilde{\beta})\right\|=\operatorname{degree}\left(f_{p_{\#}(\tilde{\beta})}\right)=\operatorname{degree}\left(f_{\tilde{\beta}^{\circ} \tilde{p}^{*}}\right)=\operatorname{degree}\left(\widetilde{p^{*}}\right) \operatorname{degree}\left(f_{\tilde{\beta}}\right)$. It follows that degree $\left(\widetilde{p^{*}}\right) \neq 0$.

Proof of Theorem 2.1. Recall the notations developed in the paragraph preceding the statement of Theorem 2.1. Recall also that we have assumed that $\pi_{1}(\widetilde{M}(\alpha))$ is cyclic.

The cover $\widetilde{M(\alpha)} \rightarrow M(\alpha)$ has branching index $k$, and so the loop $\alpha^{k}$ lifts to slopes $\tilde{\alpha}_{i}$ based at $\tilde{x}_{i}$ and lying on $\tilde{T}_{i}, i=1, \ldots, n$. Clearly, $\widetilde{M(\alpha)}$ may be identified with the Dehn filling $\tilde{M}\left(\tilde{\alpha}_{1}, \ldots, \tilde{\alpha}_{n}\right)$ of $\tilde{M}$, and so in particular, if $\langle$.$\rangle denotes normal$ closure, $\pi_{1}(\widetilde{M(\alpha)})=\tilde{\pi} /\left\langle\tilde{\alpha}_{1}, \tilde{\gamma}_{2} \tilde{\alpha}_{2} \tilde{\gamma}_{2}^{-1}, \ldots, \tilde{\gamma}_{n} \tilde{\alpha}_{n} \tilde{\gamma}_{n}^{-1}\right\rangle$. 
Suppose that $Z_{y}\left(f_{\tilde{\alpha}_{1}}\right) \leq Z_{y}\left(f_{\tilde{\beta}}\right)$ for each $\tilde{\beta} \in \tilde{L}-\{0\}$ and each $y \in \tilde{Y}_{0}$. Then $\operatorname{degree}\left(f_{\tilde{\alpha}_{1}}\right)=\min \left\{\operatorname{degree}\left(f_{\tilde{\beta}}\right) ; \tilde{\beta} \in \tilde{L}-\{0\}\right\}$. But Lemma 3.4(1) implies that

$$
\begin{aligned}
k\|\alpha\| & =\|k \alpha\|=\operatorname{degree}\left(\widetilde{p^{*}}\right) \operatorname{degree}\left(f_{\tilde{\alpha}_{1}}\right) \\
& =\operatorname{degree}\left(\widetilde{p^{*}}\right) \min \left\{\operatorname{degree}\left(f_{\tilde{\beta}}\right) ; \tilde{\beta} \in \tilde{L}-\{0\}\right\} \\
& =\min \left\{\left\|p_{\#}(\tilde{\beta})\right\| ; \quad \tilde{\beta} \in \tilde{L}-\{0\}\right\} .
\end{aligned}
$$

Note that $(d / n)=\left|L / p_{\#}(\widetilde{L})\right|$ and so, if $\eta \in L$ satisfies $\|\eta\|=s$, then $(d / n) \eta \in$ $p_{\#}(\widetilde{L})$. Thus $k\|\alpha\| \leq\|(d / n) \eta\|=(d / n) s$.

Therefore to prove the theorem, we only need to show that if $Z_{y}\left(f_{\tilde{\alpha}_{1}}\right)>Z_{y}\left(f_{\tilde{\beta}}\right)$ for some $y \in \tilde{Y}_{0}$ and some class $\tilde{\beta} \in \widetilde{L}-\{0\}$, then $k \neq 1$ and $M$ contains a closed essential surface of genus greater than 1 .

Assume first of all that $y \in Y_{0}^{\nu}$. Using Lemma 3.1, one can show that the method of $\S 1.5$ of [CGLS] applies to produce a representation $\rho \in S_{0}$ such that

(i) $t(\rho)=\nu(y)$;

(ii) the image in $P S L_{2}(\mathbf{C})$ of the group $\rho(\tilde{\pi}) \subset S L_{2}(\mathbf{C})$ is non-cyclic;

(iii) $\rho\left(\tilde{\alpha}_{1}\right)= \pm I$.

Further, Lemma 3.3 shows that for each $i=1, \ldots, n, Z_{y}\left(f_{\left[\tilde{\gamma}_{i} \tilde{\alpha}_{i} \tilde{\gamma}_{i}^{-1}\right]}\right)=Z_{y}\left(f_{\tilde{\alpha}_{1}}\right)>$ $Z_{y}\left(f_{\tilde{\beta}}\right)$ and so [CGLS, Proposition 1.5.4] guarantees that $\rho\left(\left[\tilde{\gamma}_{i} \tilde{\alpha}_{i} \tilde{\gamma}_{i}^{-1}\right]\right)= \pm I$ for each $i$. It follows that $\rho$ induces a representation with non-cyclic image $\bar{\rho}: \pi_{1}(\widetilde{M(\alpha)})=$ $\tilde{\pi} /\left\langle\tilde{\alpha}_{1}, \tilde{\gamma}_{2} \tilde{\alpha}_{2} \tilde{\gamma}_{2}^{-1}, \ldots, \tilde{\gamma}_{n} \tilde{\alpha}_{n} \tilde{\gamma}_{n}^{-1}\right\rangle \rightarrow P S L_{2}(\mathbf{C})$, which is impossible.

Assume then that $y \in \tilde{Y}_{0}-Y_{0}^{\nu}$, that is, $y$ is an ideal point of $\tilde{Y}_{0}$. According to Lemma $3.4(2)$, there is a point $x \in \tilde{X}_{0}$ with $\widetilde{p^{*}}(x)=y$. Referring back to the diagram following Lemma 3.3, we see that $x$ must be an ideal point of $\tilde{X}_{0}$. Now for each $\tilde{\delta} \in \widetilde{L}$, Lemma 3.4 shows that $Z_{x}\left(f_{p_{\#}(\tilde{\delta})}\right)=Z_{x}\left(f_{\tilde{\delta}} \widetilde{p}^{*}\right)$, and this latter quantity is equal to $b Z_{y}\left(f_{\tilde{\delta}}\right)$, where $b>0$ is the ramification index of $\widetilde{p^{*}}$ at $x$. Hence $Z_{x}\left(f_{p_{\#}(\tilde{\delta})}\right)=b Z_{y}\left(f_{\tilde{\delta}}\right)$. But then, noting that $p_{\#}\left(\alpha_{1}\right)=\alpha^{k}$, we see that $Z_{x}\left(f_{\alpha^{k}}\right)=b Z_{y}\left(f_{\tilde{\alpha}_{1}}\right)>b Z_{y}\left(f_{\tilde{\beta}}\right)=Z_{x}\left(f_{p_{\#}(\tilde{\beta})}\right)$. Hence in particular, $x$ is not a pole of $f_{\alpha^{k}}$ and thus, $x$ is not a pole of $f_{\alpha}$ (cf. Lemma 6.1). Hence $I_{\alpha}(x)$ is finite. Since we have assumed that $\alpha$ is not a strict boundary slope, [CGLS, Proposition 1.3.9] implies that $I_{\beta}(x) \neq \infty$ for all $\beta \in L$. It follows (from [CGLS, Lemma 1.6.4]) that $M$ contains a closed essential surface of genus greater than 1 .

If $k=1$, then $Z_{x}\left(f_{\alpha}\right)>Z_{x}\left(f_{p_{\#}(\tilde{\beta})}\right)$. Appealing to [CGLS, Proposition 1.6.1], we see that $M(\alpha)$ contains a closed orientable incompressible surface of genus larger than one. This is impossible as $\pi_{1}(\widetilde{M(\alpha)})$ is cyclic.

\section{Proof of Theorem 2.2}

Assume all the conditions of Theorem 2.2. The following lemma is from [CGLS, Prop. 1.5.2], to which we shall refer several times.

Lemma 4.1. Suppose that $\alpha \in L$ is a finite surgery class, and that $Z_{x}\left(f_{\alpha}\right)>$ $Z_{x}\left(f_{\beta}\right)$ for some non-zero element $\beta \in L$, and for some point $x \in X_{0}^{\nu}$. Then there is a representation $\rho \in R_{0}$ satisfying

(i) $t(\rho)=\nu(x)$;

(ii) the image in $P S L_{2}(\mathbf{C})$ of the group $\rho\left(\pi_{1}(M)\right) \subset S L_{2}(\mathbf{C})$ is non-cyclic;

(iii) $\rho(\alpha)= \pm I$. 
Therefore $\rho$ induces a representation $\bar{\rho}$ of the finite group $\pi_{1}(M(\alpha))$ onto a noncyclic subgroup of $P S L_{2}(\mathbf{C})$, and $\rho\left(\pi_{1}(M)\right)$ is a non-cyclic finite group in $S L_{2}(\mathbf{C})$.

Lemma 4.2. Suppose that $Z_{x}\left(f_{\alpha}\right)>Z_{x}\left(f_{\beta}\right)$ for some $x \in X_{0}^{\nu}$, and some nontrivial element $\beta \in L$. Let $\rho \in R_{0}$ be a representation associated to $x$, provided by Lemma 4.1. Then $\rho\left(\pi_{1}(\partial M)\right)$ is not contained in $\{ \pm I\}$.

Proof. Suppose to the contrary that $\rho\left(\pi_{1}(\partial M)\right)$ is contained in $\{ \pm I\}$, and so $Z_{x}\left(f_{\delta}\right)>0$ for any non-trivial $\delta \in L$. Recall that $\rho$ factors through a representation $\bar{\rho}$ defined on $\pi_{1}(M(\alpha))$, and that $\rho\left(\pi_{1}(M)\right)$ is a non-cyclic finite subgroup of $S L_{2}(\mathbf{C})$. Let $\widetilde{M(\alpha)} \longrightarrow M(\alpha)$ be the free regular cover associated to the homomorphism $\pi_{1}(M(\alpha)) \longrightarrow \bar{\rho}\left(\pi_{1}(M(\alpha))\right)$, and let $\tilde{M} \stackrel{p}{\longrightarrow} M$ be the restriction of this cover to $M$.

We now use the notation developed in $\S 3$. Since $\bar{\rho}\left(\pi_{1}(\partial M)\right)$ is trivial, the number of components of $\partial \tilde{M}=p^{-1}(\partial M)$ equals the degree of the covering map $p$, and thus $p_{\#}(\widetilde{L})=L$. Then $\alpha$ and $\beta$ correspond to classes $\tilde{\alpha}$ and $\tilde{\beta}$ belonging to $\widetilde{L}$. Arguing as in the proof of Theorem 2.1 (see $\S 3$ ), we have $Z_{y}\left(f_{\tilde{\alpha}}\right)>Z_{y}\left(f_{\tilde{\beta}}\right)$, where $y=\left(p^{*}\right)^{\nu}(x) \in Y_{0}^{\nu}$. As we pointed out, this inequality implies that there is a representation $\varphi \in S_{0}$ with non-cyclic image, such that $t(\varphi)=\nu(y)$. Furthermore, we showed that $\varphi$ induces a representation

$$
\bar{\varphi}: \pi_{1}(\widetilde{M(\alpha)})=\tilde{\pi} /\left\langle\tilde{\alpha}_{1}, \tilde{\gamma}_{2} \tilde{\alpha}_{2} \tilde{\gamma}_{2}^{-1}, \ldots, \tilde{\gamma}_{n} \tilde{\alpha}_{n} \tilde{\gamma}_{n}^{-1}\right\rangle \rightarrow P S L_{2}(\mathbf{C}) .
$$

Thus $\varphi$ has a finite image. But as $y=\left(p^{*}\right)^{\nu}(x)$, it follows that $t(\varphi)=t\left(\rho \circ p_{\#}\right)$, and since $\pi_{1}(\widetilde{M(\alpha)})$ is the kernel of $\bar{\rho}$, the function $t(\varphi)$ takes on values in $\{ \pm 2\}$. Then as any element in the image of $\varphi$ has finite order, we conclude that $\varphi(\tilde{\pi}) \subseteq\{ \pm I\}$, contradicting the choice of $\varphi$.

Denote by $\rho$ a representation of $R_{0}$ as guaranteed by Lemma 4.1. Since $\rho\left(\pi_{1}(M)\right)$ is a finite non-cyclic subgroup of $S L_{2}(\mathbf{C})$, we may assume, after conjugation if necessary, that $\rho\left(\pi_{1}(M)\right) \subset S U_{2}(\mathbf{C})$ (note that $\rho$ is necessarily irreducible in $S L_{2}(\mathbf{C})$ ). Further, as $\pi_{1}(\partial M)$ is an abelian subgroup of $\pi_{1}(M)$, we may arrange for $\rho\left(\pi_{1}(\partial M)\right)$ to be contained in the diagonal subgroup of $S U_{2}(\mathbf{C})$. By Lemma 4.2, $\rho\left(\pi_{1}(\partial M)\right)$ is not contained in $\{ \pm I\}$. We shall prove that

(i) $t(\rho)=\nu(x)$ is a smooth point of $X_{0}$;

(ii) $Z_{x}\left(f_{\alpha}\right)=Z_{t(\rho)}\left(f_{\alpha}\right)=2$

(iii) $Z_{x}\left(f_{\delta}\right)>0$ implies $Z_{x}\left(f_{\delta}\right) \geq 2$ for $\delta \in L$.

This will complete the proof of Theorem 2.2.

First we show that $\rho$ is a smooth point of $R_{0}$. According to [CS, 1.5.3] and [CGLS, 1.1.1], $R_{0}$ has dimension 4. Hence, $\rho$ will be a smooth point of $R_{0}$ if we can show that the Zariski tangent space of $R_{0}$ at $\rho, T_{\rho}\left(R_{0}\right)$, is also 4-dimensional ([Mu, $\S 1])$. It is well known that the Zariski tangent space to $R$ at $\rho$ includes into the set of 1-cocycles $Z^{1}\left(\pi_{1}(M) ; s l_{2}(\mathbf{C})_{A d \rho}\right)$ (see [Wl], [Gl]). Thus, we have the inequalities $4 \leq \operatorname{dim}_{\mathbf{C}} T_{\rho} R_{0} \leq \operatorname{dim}_{\mathbf{C}} Z^{1}\left(\pi_{1}(M) ; s l_{2}(\mathbf{C})_{A d \rho}\right)$. We shall show this latter quantity is precisely 4 .

The manifold $M(\alpha)$ decomposes as $M(\alpha)=M \cup_{\partial M} V(\alpha)$, where $V(\alpha)$ is a solid torus whose meridian has homology class $\alpha$ in $L$. Recall that $\rho$ induces a representation

$$
\varphi: \pi_{1}(M(\alpha)) \rightarrow A u t\left(s l_{2}(\mathbf{C})\right)
$$


and hence, associated representations $\varphi_{M}=A d \rho, \varphi_{V(\alpha)}$, and $\varphi_{\partial M}$ of $\pi_{1}(M)$, $\pi_{1}(V(\alpha))$ and $\pi_{1}(\partial M)$ to $A u t\left(s l_{2}(\mathbf{C})\right)$.

Lemma 4.3. The two inclusions $\partial M \rightarrow M$ and $\partial M \rightarrow V(\alpha)$ induce an isomorphism

$$
H^{1}\left(\pi_{1}(M) ; \varphi_{M}\right) \oplus H^{1}\left(\pi_{1}(V(\alpha)) ; \varphi_{V(\alpha)}\right) \stackrel{\cong}{\longrightarrow} H^{1}\left(\pi_{1}(\partial M) ; \varphi_{\partial M}\right) .
$$

Proof. The lemma will follow from the Mayer-Vietoris sequence for cohomology with local coefficients, associated to the decomposition $M(\alpha)=M \cup_{\partial M} V(\alpha)$, if we can show $H^{1}(M(\alpha) ; \varphi) \cong H^{2}(M(\alpha) ; \varphi) \cong 0$ (note that $M, V(\alpha)$ and $\partial M$ are aspherical, and so they may be used to calculate the cohomology of their fundamental groups). To that end, let $\widetilde{M(\alpha)}$ be the universal cover of $M(\alpha) . \widehat{M(\alpha)}$ is a homotopy 3 -sphere, since $\pi_{1}(M(\alpha))$ is finite. Hence if $j=1$ or 2 ,

$$
0=H^{j}(\widetilde{M(\alpha)} ; \mathbf{C}) \cong H^{j}\left(M(\alpha) ; \mathbf{C}\left[\pi_{1}(M(\alpha))\right]\right) \cong \oplus_{i=1}^{n} H^{j}\left(M(\alpha) ; \sigma_{i}\right)^{\operatorname{dim}_{\mathbf{C}}\left(\sigma_{i}\right)},
$$

where $\left\{\sigma_{1}, \ldots, \sigma_{n}\right\}$ is a complete collection of irreducible complex representations of $\pi_{1}(M(\alpha))$ (see [Se]). In particular, we see that $H^{j}(M(\alpha) ; \sigma) \cong 0$ for any irreducible complex representation $\sigma$ of $\pi_{1}(M(\alpha))$, and thus, $H^{j}(M(\alpha) ; \sigma) \cong 0$ for any complex representation $\sigma$ of $\pi_{1}(M(\alpha))$. Thus, $H^{1}(M(\alpha) ; \varphi) \cong H^{2}(M(\alpha) ; \varphi) \cong 0$.

Lemma 4.4. $\operatorname{dim}_{\mathbf{C}} H^{1}\left(M ; \varphi_{M}\right)=1$.

Proof. According to our hypotheses, $\varphi_{\partial M}: \pi_{1}(\partial M) \rightarrow A u t\left(s l_{2}(\mathbf{C})\right)$ is non-trivial. Further, $\varphi_{\partial M}(\alpha)=1$, and thus, $\varphi_{\partial M}$ factors through $\varphi_{V(\alpha)}$. It follows that $\varphi_{V(\alpha)}$ is also a non-trivial representation. Now any connected, finite-sheeted cover of $\partial M$ (respectively $V(\alpha)$ ) is a torus (respectively a solid torus) and thus applying [BN, Theorem 1.1 (i)], we see that $\operatorname{dim}_{\mathbf{C}} H^{1}\left(\partial M ; \varphi_{\partial M}\right)=2$, while

$$
\operatorname{dim}_{\mathbf{C}} H^{1}\left(V(\alpha) ; \varphi_{V(\alpha)}\right)=1 .
$$

Lemma 4.3 now implies $\operatorname{dim}_{\mathbf{C}} H^{1}\left(M ; \varphi_{M}\right)=1$.

Consider the $\mathbf{C}$-linear map $\delta: s l_{2}(\mathbf{C}) \rightarrow Z^{1}\left(M ; \varphi_{M}\right)$ given by $\left(\delta u_{0}\right)(\gamma)=u_{0}-$ $\varphi_{M}(\gamma)\left(u_{0}\right)=u_{0}-\rho(\gamma) u_{0} \rho(\gamma)^{-1}$. Now $\delta$ has image $B^{1}\left(M ; \varphi_{M}\right)$, and thus

$$
\begin{gathered}
\operatorname{dim}_{\mathbf{C}} Z^{1}\left(M ; \varphi_{M}\right)=\operatorname{dim}_{\mathbf{C}} H^{1}\left(M ; \varphi_{M}\right)+\operatorname{dim}_{\mathbf{C}} B^{1}\left(M ; \varphi_{M}\right) \\
=1+\left(3-\operatorname{dim}_{\mathbf{C}} \operatorname{ker}(\delta)\right)=4-\operatorname{dim}_{\mathbf{C}} k \operatorname{ker}(\delta) .
\end{gathered}
$$

It is elementary to show that $\operatorname{ker}(\delta)=0$, owing to the fact that $\rho\left(\pi_{1}(M)\right)$ is a noncyclic (therefore non-abelian) subgroup of $S U_{2}(\mathbf{C})$. Thus $\operatorname{dim}_{\mathbf{C}} Z^{1}\left(M ; \varphi_{M}\right)=4$ and so, as noted above, $\rho$ is a smooth point of $R_{0}$ (in fact a simple point of $R$ also [Sh, page 79]). The following lemma shows that $t(\rho)$ is a smooth point of $X_{0}$.

Lemma 4.5. If $\eta$ is an irreducible representation which is also a smooth point of $R_{0}$, then $t(\eta)$ is a smooth point of $X_{0}$, with $T_{t(\eta)} X_{0} \subseteq H^{1}(M ; A d \eta)$. In particular this holds for the representation $\rho$.

Proof. We first note that by [JM, Theorem 1.1], the notions of irreducible, stable and good correspond for representations of $\pi_{1}(M)$ in $S L_{2}(\mathbf{C})$ (see [JM, page 53] for the definitions of stable and good representations). Then by [JM, Proposition 1.3] for instance, the collection of irreducible representations in $R_{0}$ forms a Zariski open subset of $R_{0}$. As we have assumed that $\eta$ is irreducible, there is a Zariski open neighbourhood $U$ of $\eta$ in $R_{0}$, consisting of irreducible representations of $\pi_{1}(M)$ each of which is also a smooth point of $R_{0}$. Further, using [CS, 1.1.1], we may assume that $U$ is invariant under conjugation by elements of $S L_{2}(\mathbf{C})$. Note that by construction, 
this action induces a free action of $P S L_{2}(\mathbf{C})$ on $U$, which is also proper by [JM, Proposition 1.1]. It follows from $[\mathrm{P}]$ that every orbit in $U$ admits an analytic 2disc slice through any point, and hence, $U / P S L_{2}(\mathbf{C})$ is an analytic surface. But then using [CS, 1.5.2], one can show that $t(\eta)$ has an open neighbourhood in $X_{0}$ analytically equivalent to $U / P S L_{2}(\mathbf{C})$ (compare with the remark on page 57 of $[\mathrm{JM}])$. Thus $t(\eta)$ is a smooth point of $X_{0}$.

Finally, we note that $U \rightarrow t(U)$ is a smooth locally trivial principal $P S L_{2}(\mathbf{C})$-fibre bundle such that the inclusion $T_{\eta}\left(P S L_{2}(\mathbf{C}) \cdot \eta\right) \subset T_{\eta} R_{0} \subseteq Z^{1}(M ; A d \eta)$ corresponds to the inclusion $B^{1}(M ; A d \eta) \subset Z^{1}(M ; A d \eta)$ (see [Wl] or $\S 1$ of [Gl] for instance). Thus $T_{t(\eta)} X_{0} \subseteq H^{1}(M ; A d \eta)$.

Let $j: \partial M \rightarrow V(\alpha)$ be the inclusion, and let $j_{\#}: \pi_{1}(\partial M) \rightarrow \pi_{1}(V(\alpha))$ and

$$
j^{*}: H^{1}\left(V(\alpha) ; \varphi_{V(\alpha)}\right) \rightarrow H^{1}\left(\partial M ; \varphi_{\partial M}\right)
$$

denote the associated homomorphisms.

Lemma 4.6. If $u \in Z^{1}\left(\partial M ; \varphi_{M}\right)$, then its class in $H^{1}\left(\partial M ; \varphi_{\partial M}\right)$ lies in the image of $j^{*}$ if and only if $u(\alpha)=0$.

Proof. First note that $\varphi_{\partial M}(\alpha)=1$ implies that each coboundary in $B^{1}\left(\partial M ; \varphi_{\partial M}\right)$ vanishes on $\alpha$. Hence the value $u(\alpha) \in s l_{2}(\mathbf{C})$ depends only on the class $\bar{u}$ of $u$.

Suppose now that there is a class $\overline{u^{\prime}} \in H^{1}\left(V(\alpha) ; \varphi_{V(\alpha)}\right)$, with $\bar{u}=j^{*}\left(\overline{u^{\prime}}\right)=$ $\overline{u^{\prime} \cdot j_{\#}}$. Then $u(\alpha)=u^{\prime}\left(j_{\#}(\alpha)\right)=u^{\prime}(1)=0$.

Now assume that $u(\alpha)=0$. An easy calculation shows that $u\left(\alpha^{n}\right)=0$ for each $n \in \mathbf{Z}$, and thus, if we tentatively define $u^{\prime}: \pi_{1}(V(\alpha)) \rightarrow s l_{2}(\mathbf{C})$ by $u^{\prime}(\delta)=u(\beta)$, where $\beta \in \pi_{1}(\partial M)$ is any element with $j_{\#}(\beta)=\delta$, then $u^{\prime}$ is a well-defined 1-cocycle on $\pi_{1}(V(\alpha))$ such that $u=u^{\prime} \cdot j_{\#}$. Then $\bar{u}=j^{*}\left(\overline{u^{\prime}}\right)$.

Lemma 4.7. If $\bar{u}$ is a non-zero class in $H^{1}\left(M ; \varphi_{M}\right)$, then trace $\left(u(\alpha)^{2}\right) \neq 0$.

Proof. Let $k: \partial M \rightarrow M$ be the inclusion, and let

$$
k_{\#}: \pi_{1}(\partial M) \rightarrow \pi_{1}(M) \quad \text { and } \quad k^{*}: H^{1}\left(M ; \varphi_{M}\right) \rightarrow H^{1}\left(\partial M ; \varphi_{\partial M}\right)
$$

be the associated homomorphisms. Lemma 4.3 shows that $k^{*}(\bar{u})$ is not in the image of $j^{*}$. Hence $u(\alpha) \neq 0$ by Lemma 4.6 .

On the other hand, we claim that $u(\alpha)$ is a diagonal matrix. To see this, choose $\gamma \in \pi_{1}(\partial M)$ such that $\rho(\gamma)=\left(\begin{array}{ll}v & 0 \\ 0 & \bar{v}\end{array}\right)$, where $v^{2} \neq 1$. This is possible by our hypotheses on $\rho$. Now, $u(\alpha)+u(\gamma)=u(\alpha \gamma)=u(\gamma \alpha)=u(\gamma)+$ $\left(\begin{array}{cc}v & 0 \\ 0 & \bar{v}\end{array}\right) u(\alpha)\left(\begin{array}{cc}\bar{v} & 0 \\ 0 & v\end{array}\right)$. Hence, $u(\alpha)=\left(\begin{array}{cc}v & 0 \\ 0 & \bar{v}\end{array}\right) u(\alpha)\left(\begin{array}{cc}\bar{v} & 0 \\ 0 & v\end{array}\right)$. As $v^{2} \neq 1$, this can occur only if $u(\alpha)$ is diagonal.

We may now write $u(\alpha)=z\left(\begin{array}{cc}1 & 0 \\ 0 & -1\end{array}\right)$ for some $z \in \mathbf{C}$. Note that $z \neq 0$ as $u(\alpha) \neq 0$. Then $\operatorname{trace}\left(u(\alpha)^{2}\right)=2 z^{2} \neq 0$, and the lemma is proved.

Lemma 4.8. Let $f: X_{0} \rightarrow \mathbf{C}$ be a non-constant regular function, $x \in X_{0}$ a smooth point and $\sigma:(-\epsilon, \epsilon) \rightarrow X_{0}$ a smooth path with $\sigma(0)=x$ and $\sigma^{\prime}(0) \neq 0$. Then

$$
Z_{x}(f)=\min \left\{r ; \frac{d^{r}}{d s^{r}}(f(\sigma(s))) \neq 0\right\} .
$$


Proof. As this is a purely local question, we may assume $x=0 \in U$, where $U$ is an open subset of $\mathbf{C}$ containing $\sigma((-\epsilon, \epsilon))$. If $Z_{x}(f)=n$, we may expand $f$ near $x=0$ as $f(z)=a_{n} z^{n}+a_{n+1} z^{n+1}+\ldots$ where $a_{n} \neq 0$. Then, $f(\sigma(s))=$ $a_{n} \sigma(s)^{n}+a_{n+1} \sigma(s)^{n+1}+\ldots$. It is an elementary exercise to show that $\frac{d^{r}}{d s^{r}}(f(\sigma(s)))$ is zero for $r<n$, and non-zero for $r=n$. The proof is therefore complete.

Lemma 4.9. If $\Sigma \subset X_{0}$ denotes the singular set, then the restriction of the normalization $\nu: X_{0}^{\nu} \rightarrow X_{0}$ to $X_{0}^{\nu} \backslash \nu^{-1}(\Sigma)$ gives an equivalence between $X_{0}^{\nu} \backslash \nu^{-1}(\Sigma)$ and $X_{0} \backslash \Sigma$.

Proof. $\nu \mid: X_{0}^{\nu} \backslash \nu^{-1}(\Sigma) \rightarrow X_{0} \backslash \Sigma$ is a surjective and regular function between non-singular curves, and thus, is an open mapping with respect to the classical topologies. Let $x \in X_{0} \backslash \Sigma$, and suppose $y_{1}, y_{2} \in v^{-1}(x)$, with $y_{1} \neq y_{2}$. There are disjoint open neighbourhoods $V_{1}, V_{2}$ of $y_{1}, y_{2}$ in $X_{0}^{\nu} \backslash \nu^{-1}(\Sigma)$, and so, $\nu\left(V_{1}\right) \cap \nu\left(V_{2}\right)$ is an open neighbourhood of $x$ in $X_{0} \backslash \Sigma$. By construction, $\nu$ is a birational equivalence, and thus, $\nu \mid$ is injective off a finite subset of $X_{0}^{\nu} \backslash \nu^{-1}(\Sigma)$. But $V_{1}$ and $V_{2}$ are disjoint and it follows that $\nu\left(V_{1}\right) \cap \nu\left(V_{2}\right)$ is finite; clearly an impossibility. We conclude that $\nu^{-1}(x)$ has only one element. It follows that $\nu \mid$ is an equivalence.

Proof of Theorem 2.2. We remind the reader that we have identified the function fields $\mathbf{C}\left(X_{0}\right)$ and $\mathbf{C}\left(\tilde{X}_{0}\right)$ via the isomorphism $f \mapsto f \circ \nu$. Under this identification $f_{\alpha}$ refers to both a function defined on $X_{0}$ as well as one defined on $\tilde{X}_{0}$.

Recall that (i) $Z_{x}\left(f_{\alpha}\right)>Z_{x}\left(f_{\beta}\right)$, (ii) $\rho \in R_{0}$ is chosen as in Lemma 4.1 so that $\nu(x)=t(\rho) \in X_{0}$, and (iii) $\rho\left(\pi_{1}(\partial M)\right)$ is not contained in $\{ \pm I\}$. According to Lemma 4.5, $t(\rho)$ is a smooth point of $X_{0}$, and so part (1) of Theorem 2.2 holds. Furthermore, by Lemma 4.9 we see that $Z_{x}\left(f_{\alpha}\right)=Z_{x}\left(f_{\alpha} \cdot \nu\right)=Z_{\nu(x)}\left(f_{\alpha}\right)$. Thus we may compute $Z_{\nu(x)}\left(f_{\alpha}\right)$ in $X_{0}$.

Consider now $\bar{u}$, a non-zero class in $H^{1}\left(M ; \varphi_{M}\right)=T_{\nu(x)} X_{0}$ (Lemma 4.4 and Lemma 4.5). We have seen that $\rho$ is a smooth point of $R_{0}$, and thus, the tangent space of $R_{0}$ at $\rho$ can be identified with $Z^{1}\left(M ; \varphi_{M}\right)$. It follows that there is a smooth curve in $R_{0}$ of the form

$$
\rho_{s}=\exp \left(s u+s^{2} u_{2}+O\left(s^{3}\right)\right) \rho
$$

where $u_{2}: \pi_{1}(M) \rightarrow s l_{2}(\mathbf{C})$ is defined for $s$ close to 0 . Then, if we set $\sigma(s)=t\left(\rho_{s}\right)$, we obtain a smooth curve in $X_{0}$ with $\sigma(0)=\nu(x)$, and $\sigma^{\prime}(0)=\bar{u} \in H^{1}\left(M ; \varphi_{M}\right)=$ $T_{\nu(x)} X_{0}$.

Now, $\rho(\alpha)=\epsilon I$ for some $\epsilon= \pm 1$, and thus, $\operatorname{trace}\left(\rho_{s}(\alpha)\right)=\operatorname{trace}(\exp (\operatorname{su}(\alpha)+$ $\left.\left.s^{2} u_{2}(\alpha)+O\left(s^{3}\right)\right) \rho(\alpha)\right)=\epsilon \operatorname{trace}\left(I+s u(\alpha)+s^{2}\left(u_{2}(\alpha)+\frac{1}{2} u(\alpha)^{2}\right)+O\left(s^{3}\right)\right)=2 \epsilon+$ $\epsilon\left(\operatorname{trace}\left(u(\alpha)^{2}\right) / 2\right) s^{2}+O\left(s^{3}\right)$. Hence,

$$
f_{\alpha}(\sigma(s))=\left[\operatorname{trace}\left(\rho_{s}(\alpha)\right)\right]^{2}-4=2 \operatorname{trace}\left(u(\alpha)^{2}\right) s^{2}+O\left(s^{3}\right) .
$$

Lemmas 4.7 and 4.8 now show that $Z_{\nu(x)}\left(f_{\alpha}\right)=2$.

Finally, we show that for $\delta \in L, Z_{\nu(x)}\left(f_{\delta}\right)>0$ implies $Z_{\nu(x)}\left(f_{\delta}\right) \geq 2$. Since $Z_{\nu(x)}\left(f_{\delta}\right)>0, \rho(\delta)$ is conjugate to $\pm I$. Expanding $f_{\delta}(\sigma(s))$ as above, we see $f_{\delta}(\sigma(s))=2 \operatorname{trace}\left(u(\delta)^{2}\right) s^{2}+O\left(s^{3}\right)$. Hence $Z_{\nu(x)}\left(f_{\delta}\right) \geq 2$. 


\section{Proof of Theorem 2.3}

To prove the theorem, we need several preparatory lemmas concerning the six types of finite non-cyclic groups discussed in the introduction, and their representations into $S L_{2}(\mathbf{C}) . H_{*}(M)$ will denote homology groups with integer coefficients of a manifold $M$, and $H_{1}(G)$ will denote the abelianization of a group $G$.

Lemma 5.1. Let $M$ be a compact, connected, orientable, irreducible 3-manifold with $\partial M$ a torus. Suppose that $M(\alpha)$ has finite fundamental group. Then $H_{1}(M(\alpha))$ and $H_{1}(M)$ are given according to the following table:

\begin{tabular}{|c|c|c|}
\hline$\pi_{1}(M(\alpha))$ & $H_{1}(M(\alpha))$ & $H_{1}(M)$ \\
\hline $\mathbf{Z}_{j}$ & $\mathbf{Z}_{j}$ & $\mathbf{Z} \oplus \mathbf{Z}_{q}, q \mid j$ \\
\hline$D_{4 n} \times \mathbf{Z}_{j}, n$ even & $\mathbf{Z}_{2} \oplus \mathbf{Z}_{2 j}$ & $\mathbf{Z} \oplus \mathbf{Z}_{2} \oplus \mathbf{Z}_{2^{p}}, 0 \leq p \leq 1, q \mid j$ \\
\hline$D\left(2^{k}, 2 l+1\right) \times \mathbf{Z}_{j}$ & $\mathbf{Z}_{2^{k} j}$ & $\mathbf{Z} \oplus \mathbf{Z}_{2^{p} q}, 0 \leq p \leq k, q \mid j$ \\
\hline$T\left(8,3^{k}\right) \times \mathbf{Z}_{j}$ & $\mathbf{Z}_{3^{k}}$ & $\mathbf{Z} \oplus \mathbf{Z}_{3^{p} q}, 0 \leq p \leq k, q \mid j$ \\
\hline$O_{48} \times \mathbf{Z}_{j}$ & $\mathbf{Z}_{2 j}$ & $\mathbf{Z} \oplus \mathbf{Z}_{2^{p} q}, 0 \leq p \leq 1, q \mid j$ \\
\hline$I_{120} \times \mathbf{Z}_{j}$ & $\mathbf{Z}_{j}$ & $\mathbf{Z} \oplus \mathbf{Z}_{q}, q \mid j$ \\
\hline$Q(8 n, k, l) \times \mathbf{Z}_{j}$ & $\mathbf{Z}_{2} \oplus \mathbf{Z}_{2 j}$ & $\mathbf{Z} \oplus \mathbf{Z}_{2} \oplus \mathbf{Z}_{2^{p} q}, 0 \leq p \leq 1, q \mid j$ \\
\hline
\end{tabular}

Proof. The proof relies on elementary homological arguments. We observe first of all that $H_{1}(M(\alpha))$ results from $H_{1}(M)$ by adding a single relation. Thus, the rank of $H_{1}(M)$ is necessarily no larger than 1 . But it is at least 1 by [Hl, Lemma 6.7]. Furthermore, as the abelianization of $\pi_{1}(M(\alpha))$ may be determined from the presentations listed in the introduction, the observation above allows the determination of the torsion part of $H_{1}(M)$ as stated in the lemma. We omit the details.

Lemma 5.2. (1) An odd D-type group has a unique index two normal subgroup. This subgroup is a cyclic group.

(2) An even D-type group has an index two normal cyclic subgroup.

(3) A Q-type group contains a unique index four normal subgroup. This subgroup is a cyclic group.

(4) An O-type group contains a unique index two normal subgroup. This subgroup is a T-type group.

Proof. (1) For an odd $D$-type group $D\left(2^{k}, 2 l+1\right) \times \mathbf{Z}_{j}=\left\{x, y ; x^{2^{k}}=1, y^{2 l+1}=\right.$ $\left.1, x y x^{-1}=y^{-1}\right\} \times \mathbf{Z}_{j}$, the subgroup generated by $x^{2}, y$ and $\mathbf{Z}_{j}$ is cyclic and has index two (thus, it is normal). The uniqueness follows by considering the abelianization of the $D$-type group.

(2) For an even $D$-type group $D_{4 n} \times \mathbf{Z}_{j}=\left\{x, y ; x^{2}=(x y)^{2}=y^{n}\right\} \times \mathbf{Z}_{j}(n$ even), the subgroup generated by $y$ and $\mathbf{Z}_{j}$ is cyclic and has index two.

(3) For a $Q$-type group $Q(8 n, k, l) \times Z_{j}=\left\{x, y, z ; x^{2}=(x y)^{2}=y^{2 n}, z^{k l}=\right.$ $\left.1, x z x^{-1}=z^{r}, y z y^{-1}=z^{-1}\right\} \times \mathbf{Z}_{j}$, the subgroup generated by $y^{2}, z$ and $\mathbf{Z}_{j}$ is cyclic and has index four. In fact, it is the kernel of the surjective homomorphism $Q(8 n, k, l) \times Z_{j} \rightarrow H_{1}(Q(8 n, k, l))=\mathbf{Z}_{2} \oplus \mathbf{Z}_{2}$. As any group of order 4 is abelian, the uniqueness follows.

(4) The subgroup is the kernel of the surjective homomorphism $O_{48} \times \mathbf{Z}_{j} \rightarrow$ $H_{1}\left(O_{48}\right)=\mathbf{Z}_{2}$. Note that the commutator subgroup of $O_{48}$ is $T_{24}$. 
We recall some well known facts: every finite subgroup of $P S L_{2}(\mathbf{C})$ is conjugate in $P S L_{2}(\mathbf{C})$ to a subgroup of $P S U(2) \cong S O(3)$ ([Wo, Lemma 4.7.1]); the only finite subgroups of $P S L_{2}(\mathbf{C})$ are cyclic groups $\mathbf{Z}_{j}$, dihedral groups $\bar{D}_{2 n}$ (of order $2 n$ ), the tetrahedral group $\bar{T}_{12}$, the octahedral group $\bar{O}_{24}$ and the icosahedral group $\bar{I}_{60}$ ([Wo, Lemma 2.6.5]); two finite subgroups of $P S L_{2}(\mathbf{C})$ are conjugate iff they are isomorphic ([Wo, Lemma 2.6.5]). Hence for a finite group $G$, if $\varphi: G \rightarrow P S L_{2}(\mathbf{C})$ is a representation, then we may assume, up to conjugation, that $\varphi(G) \subset P S U(2)$; if $\varphi_{1}, \varphi_{2}: G \rightarrow P S L_{2}(\mathbf{C})$ are two representations with $\varphi_{1}(G)$ isomorphic to $\varphi_{2}(G)$, then we may assume, up to conjugation, that $\varphi_{1}(G)=\varphi_{2}(G)$.

Lemma 5.3. (1) Let $G$ be a T-type group. Then there is, up to conjugation, exactly one irreducible representation $\varphi: G \rightarrow P S L_{2}(\mathbf{C})$. The representation has image $\varphi(G)=\bar{T}_{12}$.

(2) Let $G$ be an $O$-type group. Then there are, up to conjugation, exactly two irreducible representations $\varphi: G \rightarrow P S L_{2}(\mathbf{C})$. One of the two representations has image $\bar{O}_{24}$ and the other has image $\bar{D}_{6}$.

(3) Let $G$ be an I-type group. Then there are, up to conjugation, exactly two irreducible representations $\varphi: G \rightarrow P S L_{2}(\mathbf{C})$. The two representations differ by the outer-automorphism of $\bar{I}_{60}$. Both representations have image $\bar{I}_{60}$.

Proof. We only need to prove the lemma in $P S U(2)$.

(1) Suppose that $G=T\left(8,3^{k}\right) \times \mathbf{Z}_{j}$, and recall that $T\left(8,3^{k}\right)$ is presented by $\left\{x, y, z ; x^{2}=(x y)^{2}=y^{2}, z^{3^{k}}=1, z x z^{-1}=y, z y z^{-1}=x y\right\}$. Let $h$ be a generator of $\mathbf{Z}_{j}$ and suppose that $\varphi: G \rightarrow P S U(2)$ is an irreducible representation. Now $\varphi(G)$ is a finite non-abelian subgroup of $P S U(2)$ which cannot be a dihedral group or $\bar{O}_{24}$, since $H_{1}(G)=\mathbf{Z}_{3^{k} j}$ has odd order $3^{k} j$. As $x^{2}, y^{2}$ and $h$ are central elements in $G, \varphi\left(x^{2}\right)=\varphi\left(y^{2}\right)=\varphi(h)=1$. Therefore, $\varphi(G)$ has no element of order 5 , and thus it cannot be $\bar{I}_{60}$. We conclude that $\varphi(G)$ is isomorphic to $\bar{T}_{12}$, which implies that $\varphi(z)$ has order 3 , while $\varphi(x)$ and $\varphi(y)$ each have order 2 (for otherwise $\varphi(G)$ would be cyclic). Now up to conjugation, we may assume that $\varphi(z)= \pm\left(\begin{array}{cc}\xi & 0 \\ 0 & \bar{\xi}\end{array}\right)$ where $\xi=e^{2 \pi i / 3}$, and that $\varphi(x)= \pm B\left(\begin{array}{cc}i & 0 \\ 0 & -i\end{array}\right) B^{-1}$ for some $B \in S U(2, C)$. Hence, $\varphi(x)= \pm\left(\begin{array}{cc}t i & b \\ -\bar{b} & -t i\end{array}\right)$ for some $t \in \mathbf{R}, b \in \mathbf{C}, t^{2}+|b|^{2}=1$.

Referring back to the presentation of $T\left(8,3^{k}\right)$ we see that

$$
\varphi(y)= \pm\left(\begin{array}{cc}
\xi & 0 \\
0 & \bar{\xi}
\end{array}\right)\left(\begin{array}{cc}
t i & b \\
-\bar{b} & -t i
\end{array}\right)\left(\begin{array}{cc}
\bar{\xi} & 0 \\
0 & \xi
\end{array}\right)= \pm\left(\begin{array}{cc}
t i & b \xi^{2} \\
-\bar{b} \bar{\xi}^{2} & -t i
\end{array}\right)
$$

and so

$$
\varphi(x y)= \pm\left(\begin{array}{cc}
t i & b \\
-\bar{b} & -t i
\end{array}\right)\left(\begin{array}{cc}
t i & b \xi^{2} \\
-\bar{b} \bar{\xi}^{2} & -t i
\end{array}\right)= \pm\left(\begin{array}{cc}
-t^{2}-|b|^{2} \bar{\xi}^{2} & t b i\left(\xi^{2}-1\right) \\
-t \bar{b} i\left(\bar{\xi}^{2}-1\right) & -t^{2}-|b|^{2} \xi^{2}
\end{array}\right) .
$$

But $0=\operatorname{trace}(\varphi(x y))=-2 t^{2}+|b|^{2}$. Thus $3 t^{2}=1, t= \pm 1 / \sqrt{3}$ and $b=\sqrt{2 / 3} e^{i \theta}$ for some $\theta \in[0,2 \pi)$. 
Hence, up to conjugation,

$$
\begin{aligned}
& \{\varphi(z), \varphi(x)\}=\left\{ \pm\left(\begin{array}{cc}
\xi & 0 \\
0 & \bar{\xi}
\end{array}\right), \pm\left(\begin{array}{cc}
i / \sqrt{3} & \sqrt{2 / 3} e^{i \theta} \\
-\sqrt{2 / 3} e^{-i \theta} & -i / \sqrt{3}
\end{array}\right)\right\} \\
& =\left(\begin{array}{cc}
e^{\theta i / 2} & 0 \\
0 & e^{-\theta i / 2}
\end{array}\right)\left\{ \pm\left(\begin{array}{cc}
\xi & 0 \\
0 & \bar{\xi}
\end{array}\right), \pm\left(\begin{array}{cc}
i / \sqrt{3} & \sqrt{2 / 3} \\
-\sqrt{2 / 3} & -i / \sqrt{3}
\end{array}\right)\right\} \\
& \times\left(\begin{array}{cc}
e^{-\theta i / 2} & 0 \\
0 & e^{\theta i / 2}
\end{array}\right) \\
& =\left(\begin{array}{cc}
e^{(\theta-\pi) i / 2} & 0 \\
0 & e^{-(\theta-\pi) i / 2}
\end{array}\right)\left\{ \pm\left(\begin{array}{cc}
\xi & 0 \\
0 & \bar{\xi}
\end{array}\right), \pm\left(\begin{array}{cc}
i / \sqrt{3} & -\sqrt{2 / 3} \\
\sqrt{2 / 3} & -i / \sqrt{3}
\end{array}\right)\right\} \\
& \times\left(\begin{array}{cc}
e^{-(\theta-\pi) i / 2} & 0 \\
0 & e^{(\theta-\pi) i / 2}
\end{array}\right) .
\end{aligned}
$$

Consequently, there is, up to conjugation, at most one irreducible representation $\varphi: G \rightarrow P S U(2)$. It is easy to check that $\left\{ \pm\left(\begin{array}{cc}\xi & 0 \\ 0 & \bar{\xi}\end{array}\right), \pm\left(\begin{array}{cc}i / \sqrt{3} & \sqrt{2 / 3} \\ -\sqrt{2 / 3} & -i / \sqrt{3}\end{array}\right)\right\}$ does generate a group isomorphic to $\bar{T}_{12}$.

(2) and (3) can be proved similarly. We omit the details.

Lemma 5.4. Let $G$ be a finite non-cyclic group which is the fundamental group of a 3-manifold. Let $\rho: G \rightarrow S L_{2}(\mathbf{C})$ be an irreducible representation and let $\bar{\rho}$ : $G \rightarrow P S L_{2}(\mathbf{C})$ be the associated representation. Then ker $(\bar{\rho})$ is cyclic, except when $G=O_{48} \times \mathbf{Z}_{j}$ and image $(\bar{\rho})=\bar{D}_{6}$, in which case $\operatorname{ker}(\bar{\rho})=D_{8} \times \mathbf{Z}_{j}$.

Proof. (1) Suppose $G=T\left(8,3^{k}\right) \times \mathbf{Z}_{j}=\left\{x, y, z ; x^{2}=(x y)^{2}=y^{2}, z^{3^{k}}=1, z x z^{-1}=\right.$ $\left.y, z y z^{-1}=x y\right\} \times \mathbf{Z}_{j}$. The cyclic subgroup of $G$ generated by $z^{3}, x^{2}$ and $\mathbf{Z}_{j}$ has order $(2) 3^{k-1} j$ and is contained in $\operatorname{ker}(\bar{\rho})$, while $\bar{\rho}(G)=\bar{T}_{12}$ by Lemma 5.3 (1), therefore $\operatorname{ker}(\bar{\rho}) \cong \mathbf{Z}_{(2) 3^{k-1} j}$.

(2) Suppose $G=D\left(2^{k}, 2 l+1\right) \times \mathbf{Z}_{j}$. Now, any proper normal subgroup of $G$ lies in the index 2 cyclic subgroup described in Lemma $5.2(1)$, and therefore $k e r(\bar{\rho})$ is cyclic.

(3) Suppose $G=D_{4 n} \times \mathbf{Z}_{j}$. Then, since the only normal subgroups of index greater than 2 in $G$ lie in $\left[D_{4 n}, D_{4 n}\right] \times \mathbf{Z}_{j}=\mathbf{Z}_{2 n j}, k e r(\bar{\rho})$ is cyclic.

(4) Suppose $G=I_{120} \times \mathbf{Z}_{j}$. Then $\bar{\rho}(G)=\bar{I}_{60}$ by Lemma 5.3 (3), and thus, $\operatorname{ker}(\bar{\rho})=\mathbf{Z}_{2 j}$.

(5) Suppose that $G=Q(8 n, k, l) \times \mathbf{Z}_{j}=\left\{x, y, z ; x^{2}=(x y)^{2}=y^{2} n, z^{k l}=\right.$ $\left.1, x z x^{-1}=z^{r}, y z y^{-1}=z^{-1}\right\} \times \mathbf{Z}_{j}$. Recall that $n, k, l$ are mutually relative prime integers, and $r \equiv-1(\bmod k), r \equiv 1(\bmod l)$.

(a) If $\bar{\rho}(z)=1$, then $\bar{\rho}$ factors through $D_{4 n}=\left\langle x, y, z ; x^{2}=(x y)^{2}=y^{2 n}\right\rangle$ :

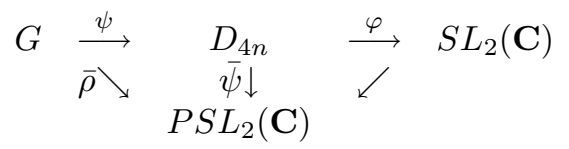

Note that $k e r(\psi) \cong \mathbf{Z}_{j k l}$, generated by $z$ and $\mathbf{Z}_{j}$. Now case (3) shows $k e r(\bar{\varphi})$ is cyclic and indeed, it is a subgroup of $\left\{1, y^{2}, \ldots, y^{2(n-1)}\right\}$. Then $\operatorname{ker}(\bar{\rho})=\psi^{-1}(\operatorname{ker}(\varphi)) \subset$ $\left\langle y^{2}, z\right\rangle \times \mathbf{Z}_{j} \cong \mathbf{Z}_{2 n k l j}$.

(b) If $\bar{\rho}(z) \neq 1$, then $\bar{\rho}(z)^{2} \neq 1$ as $\bar{\rho}(z)$ has odd order. The relation $y z y^{-1}=z^{-1}$ implies $\bar{\rho}(y)$ has order 2. Set $A=\rho(x), B=\rho(y), C=\rho(z) \in S U(2) \subset S L_{2}(\mathbf{C})$. 
Then we assume, after a possible conjugation, that $B=\left(\begin{array}{cc}0 & 1 \\ -1 & 0\end{array}\right)$. If $A=$ $\left(\begin{array}{ll}a & b \\ c & d\end{array}\right)$, then the relation $x^{2}=(x y)^{2}$ implies

$$
\left(\begin{array}{ll}
a & b \\
c & d
\end{array}\right)=\left(\begin{array}{cc}
0 & 1 \\
-1 & 0
\end{array}\right)\left(\begin{array}{ll}
a & b \\
c & d
\end{array}\right)\left(\begin{array}{cc}
0 & 1 \\
-1 & 0
\end{array}\right)=\left(\begin{array}{cc}
-d & c \\
b & -a
\end{array}\right) .
$$

Hence, $a=-d, b=c$ and so, $A=\left(\begin{array}{cc}a & b \\ b & -a\end{array}\right)$. Thus $A$ has order 4 , and $\bar{\rho}(x)$ has order 2. Also $A B=\left(\begin{array}{cc}-b & a \\ a & b\end{array}\right)$ has order 4 and thus $\bar{\rho}(x y)$ has order 2. Now it is clear that the abelianization of $\bar{\rho}(G)$ is generated by $\bar{\rho}(x)$ and $\bar{\rho}(y)$, and is isomorphic to $\mathbf{Z}_{\mathbf{2}} \oplus \mathbf{Z}_{2}$. It follows that $\operatorname{ker}(\bar{\rho})$ is contained in the kernel of the onto map $G \rightarrow H_{1}(Q(8 n, k, l))=\mathbf{Z}_{\mathbf{2}} \oplus \mathbf{Z}_{2}$, and thus, is cyclic by Lemma $5.2(3)$.

(6) Suppose $G=O_{48} \times \mathbf{Z}_{j}$. Then, $\operatorname{ker}(\bar{\rho})=\mathbf{Z}_{2 j}$ if $\bar{\rho}(G)=\bar{O}_{24}$, or $\operatorname{ker}(\bar{\rho})=$ $D_{8} \times \mathbf{Z}_{j}$ if $\bar{\rho}(G)=\bar{D}_{6}($ cf. Lemma $5.3(2)$ ).

Lemma 5.5. Let $M$ be a compact, connected, orientable, irreducible 3-manifold $M$ with $\partial M$ a torus.

(1) If $\rho \in R=H o m\left(\pi_{1}(M), S L_{2}(\mathbf{C})\right)$, then $\epsilon \rho \in R$ for any $\epsilon \in \operatorname{Hom}\left(\pi_{1}(M), \mathbf{Z}_{2}\right)$, where $\epsilon \rho: \pi_{1}(M) \rightarrow S L_{2}(\mathbf{C})$ is defined by $(\epsilon \rho)(\gamma)=\epsilon(\gamma) \rho(\gamma)$.

(2) Suppose that $\rho_{i} \in R$ is a lift of $\bar{\rho}_{i} \in \operatorname{Hom}\left(\pi_{1}(M), P S L_{2}(\mathbf{C})\right)$, for $i=1$, 2. If $\bar{\rho}_{1}$ is equivalent to $\bar{\rho}_{2}$, then $\rho_{1}$ is equivalent to $\epsilon \rho_{2}$, for some $\epsilon \in \operatorname{Hom}\left(\pi_{1}(M), \mathbf{Z}_{2}\right)$.

(3) $\rho \in R$ with finite image is equivalent to $\epsilon \rho$ in $S L_{2}(\mathbf{C})$ for some non-trivial $\epsilon \in \operatorname{Hom}\left(\pi_{1}(M), \mathbf{Z}_{2}\right)$ iff $\rho\left(\pi_{1}(M)\right)=D_{4 n}$ for some $n \geq 1$.

Proof. It is elementary to verify (1) and (2). We give the proof of (3).

$(\Rightarrow)$ Suppose $\epsilon \rho=A \rho A^{-1}$ for some $A \in S L_{2}(\mathbf{C})$. Let $\xi$ be an element of $\pi_{1}(M)$ such that $\epsilon(\xi)=-I$. Set $\rho(\xi)=B$. Then $-B=\epsilon(\xi) \rho(\xi)=A \rho(\xi) A^{-1}=$ $A B A^{-1}$, and so $A=-B A B^{-1}, B=-A B A^{-1}$ and $-A B=B A$. It follows that $\operatorname{trace}(A)=\operatorname{trace}(B)=\operatorname{trace}(A B)=0$. Without loss of generality, we may assume $A=\left(\begin{array}{cc}i & 0 \\ 0 & -i\end{array}\right)$ and $B=\left(\begin{array}{cc}a & b \\ c & -a\end{array}\right)$. Then, $0=\operatorname{trace}(A B)=2 i a$ and thus, $B=\left(\begin{array}{cc}0 & b \\ -b^{-1} & 0\end{array}\right)$ for some $b \in \mathbf{C}^{*}$. Conjugating by $\left(\begin{array}{cc}1 / \sqrt{b} & 0 \\ 0 & \sqrt{b}\end{array}\right)$, we may assume $A=\left(\begin{array}{cc}i & 0 \\ 0 & -i\end{array}\right)$ and $B=\left(\begin{array}{cc}0 & 1 \\ -1 & 0\end{array}\right)$.

Set $\tilde{\pi}=\operatorname{ker}(\epsilon)$. Then $\tilde{\pi}$ is an index two subgroup of $\pi_{1}(M)$. For $\gamma \in \tilde{\pi}$, $\rho(\gamma)=A \rho(\gamma) A^{-1}$, and so it follows that $\rho(\gamma)$ is diagonal. Thus, the finite group $\rho(\tilde{\pi})$ includes in $D=\left\{\left(\begin{array}{cc}a & 0 \\ 0 & a^{-1}\end{array}\right) ; a \in \mathbf{C}^{*}\right\}$, implying that it is in fact a cyclic group. As $\pi_{1}(M)$ is generated by $\tilde{\pi}$ and $\xi, \rho\left(\pi_{1}(M)\right)$ is generated by $\rho(\tilde{\pi})$ and $\rho(\xi)=\left(\begin{array}{cc}0 & 1 \\ -1 & 0\end{array}\right)$. Thus, $\rho\left(\pi_{1}(M)\right)=D_{4 n}$ for some $n \geq 1$.

$(\Leftarrow)$ Suppose that $\rho\left(\pi_{1}(M)\right)=D_{4 n}$ for some $n \geq 1$. Note that $\rho\left(H_{1}(M)\right)=$ $H_{1}\left(D_{4 n}\right)$, and that $D_{4 n}$ contains a unique index 2 cyclic normal subgroup $\mathbf{Z}_{2 n}$ (Lemma 5.2). Hence, $\tilde{\pi}=\rho^{-1}\left(\mathbf{Z}_{2 n}\right)$ is an index two subgroup of $\pi_{1}(M)$, and there is a non-trivial element $\epsilon \in \operatorname{Hom}\left(\pi_{1}(M), \mathbf{Z}_{2}\right)$ such that $\epsilon(\tilde{\pi})=I$. Let $\xi \in \pi_{1}(M)$ such that $\epsilon(\xi)=-I$. 
We may assume that $\rho\left(\pi_{1}(M)\right) \subset S U(2)$, and further, that $\rho(\tilde{\pi}) \subset S^{1}=$ $\left\{\left(\begin{array}{cc}a & 0 \\ 0 & \bar{a}\end{array}\right) ;|a|=1\right\}$. Then necessarily, $\rho\left(\pi_{1}(M)\right)=D_{4 n} \subset S^{1} \cup\left\{\left(\begin{array}{cc}0 & b \\ -\bar{b} & 0\end{array}\right)\right.$; $|b|=1\}$, and we may assume that $\rho(\xi) \in\left\{\left(\begin{array}{cc}0 & b \\ -\bar{b} & 0\end{array}\right) ;|b|=1\right\}$. When $n \geq 2$ this is clear, while when $n=1$, we note that $D_{4}=\mathbf{Z}_{4}$ is generated by $\rho(\xi)$, and the condition is easily satisfied. Therefore, after a possible conjugation, we have $\rho(\xi)=\left(\begin{array}{cc}0 & 1 \\ -1 & 0\end{array}\right)$. Finally, if we set $A=\left(\begin{array}{cc}i & 0 \\ 0 & -i\end{array}\right)$, it is easy to check that $\epsilon \rho=A \rho A^{-1}$.

In Lemma 5.5, if $H_{1}(M)=\mathbf{Z} \oplus \mathbf{Z}_{p}$ with $p$ odd, then there is a unique nontrivial $\epsilon \in \operatorname{Hom}\left(\pi_{1}(M), \mathbf{Z}_{2}\right)$. Thus for each $\rho \in R$ with $\rho\left(\pi_{1}(M)\right)$ finite, if $\rho(\pi)$ is not a dihedral group, then there are exactly two lifts in $R, \rho$ and $\epsilon \rho$, of $\bar{\rho}$ : $\pi(M) \rightarrow P S L_{2}(\mathbf{C})$; and if $\rho(\pi)$ is a dihedral group (necessarily an odd dihedral group by Lemma 5.1), then $\rho$ is equivalent to $\epsilon \rho$.

In Lemma 5.5, if $H_{1}(M)=\mathbf{Z} \oplus \mathbf{Z}_{p}$ with $p>0$ even, then there are three nontrivial elements in $\operatorname{Hom}\left(\pi_{1}(M), \mathbf{Z}_{2}\right)$, say $\epsilon_{i}, i=1,2,3$. Thus for each $\rho \in R(M)$ with $\rho(\pi)$ finite, if $\rho(\pi)$ is not a dihedral group, then there are exactly four lifts in $R, \rho$ and $\epsilon_{i} \rho, i=1,2,3$, of $\bar{\rho}: \pi_{1}(M) \rightarrow P S L_{2}(\mathbf{C})$; and if $\rho(\pi)=D_{4 n}$ is a dihedral group with $n \geq 3$, then it can be easily checked that there are exactly two lifts in $R$ of $\bar{\rho}: \pi_{1}(M) \rightarrow P S L_{2}(\mathbf{C})$.

We now proceed to prove Theorem 2.3. Henceforth in this section, we shall assume all the conditions of Theorem 2.3: $M$ a compact, connected, orientable, hyperbolic 3-manifold, with $\partial M$ a torus; $s=\min \left\{\|\delta\| ; \delta \in L=H_{1}(M), \delta \neq 0\right\}$; $\alpha \in L$ a finite surgery class which is not a strict boundary class. Appealing to $\S 1$ of [CGLS], we may further assume that $\alpha$ is a non-cyclic finite surgery class. Let $\theta \in L$ be a class with $\|\theta\|=s$.

Lemma 5.6. Let $\beta \in L$ be a non-trivial element.

(1) If $\alpha$ is a T-type surgery class, then there are at most 2 points $x \in X_{0}^{\nu}$ such that $Z_{x}\left(f_{\alpha}\right)>Z_{x}\left(f_{\beta}\right)$.

(2) If $\alpha$ is an I-type surgery class, then there are at most 4 points $x \in X_{0}^{\nu}$ such that $Z_{x}\left(f_{\alpha}\right)>Z_{x}\left(f_{\beta}\right)$.

(3) If $\alpha$ is an $O$-type surgery class, then there are at most 3 points $x \in X_{0}^{\nu}$ such that $Z_{x}\left(f_{\alpha}\right)>Z_{x}\left(f_{\beta}\right)$ if $H_{1}(M)$ has no non-trivial even torsion, and there are at most 6 points $x \in X_{0}^{\nu}$ such that $Z_{x}\left(f_{\alpha}\right)>Z_{x}\left(f_{\beta}\right)$ if $H_{1}(M)$ has non-trivial even torsion.

Proof. (1) Suppose that $Z_{x_{i}}\left(f_{\alpha}\right)>Z_{x_{i}}\left(f_{\beta}\right)$ for two distinct points $x_{i} \in X_{0}^{\nu}, i=$ 1,2. Recall that if $\rho_{i} \in R_{0}$ is a representation associated to $x_{i}$, as provided by Lemma 4.1, then $\bar{\rho}_{i}$ is an irreducible representation of $\pi_{1}(M(\alpha))$ in $P S L_{2}(\mathbf{C})$. Now Lemma 5.3 (1) shows that $\bar{\rho}_{1}$ and $\bar{\rho}_{2}$ are equivalent in $P S L_{2}(\mathbf{C})$ and hence, appealing to Lemma 5.5 and the remarks which follow it, we deduce that $\rho_{2}$ is equivalent to $\epsilon \rho_{1}$. Next, by applying Lemma 4.5 it follows that $\nu\left(x_{i}\right)=t\left(\rho_{i}\right)$ is a smooth point of $X_{0}$, and therefore, Lemma 4.9 implies that $x_{1}$ and $x_{2}$ are the only points in $X_{0}^{\nu}$ satisfying $Z_{x}\left(f_{\alpha}\right)>Z_{x}\left(f_{\beta}\right)$.

The proofs of (2) and (3) are similar to that of (1).

Corollary 5.7. (1) If $\alpha$ is a T-type surgery class, then $\|\alpha\| \leq s+4$.

(2) If $\alpha$ is an I-type surgery class, then $\|\alpha\| \leq s+8$. 
(3) If $\alpha$ is an O-type surgery class, then $\|\alpha\| \leq s+6$ if $H_{1}(M)$ has no non-trivial even torsion, and $\|\alpha\| \leq s+12$ if $H_{1}(M)$ has non-trivial even torsion.

Proof. Refer to Theorem 2.2 and Lemma 5.6.

Proof of Theorem 2.3. (1) This is Corollary 1.1.4 of [CGLS].

(2) Suppose that $\pi_{1}(M(\alpha))$ is a $D$-type group. By Lemma $5.2(1-2), M(\alpha)$ has a free double cover $\widetilde{M(\alpha)}$ which has cyclic fundamental group. Hence, the conclusion follows from Theorem 2.1.

Suppose that $\pi_{1}(M(\alpha))$ is a $Q$-type group. By Lemma $5.2(3), M(\alpha)$ has a free regular 4-sheeted cover $\widetilde{M(\alpha)}$ which has cyclic fundamental group, the kernel of the composition $\pi_{1}(M(\alpha)) \rightarrow H_{1}(M(\alpha)) \rightarrow \mathbf{Z}_{2} \oplus \mathbf{Z}_{2}$. By Theorem 2.1, we only need to show that $\tilde{M}$ has at least two boundary components. But this is true because the homomorphism $\pi_{1}(\partial M) \rightarrow \pi_{1}(M(\alpha)) \rightarrow H_{1}(M(\alpha)) \rightarrow \mathbf{Z}_{2} \oplus \mathbf{Z}_{2}$ factors through the arrow $H_{1}\left(\partial M ; \mathbf{Z}_{2}\right) \rightarrow H_{1}\left(M ; \mathbf{Z}_{2}\right)$, and hence has cyclic image. Thus, it is not onto.

(3) The inequality (i) comes from Corollary 5.7. For part (ii), we may assume $\|\alpha\|>s$. Hence, there is a point $x \in X_{0}^{\nu}$ such that $Z_{x}\left(f_{\alpha}\right)>Z_{x}\left(f_{\theta}\right)$. Let $\rho$ be an associated representation as provided by Lemma 4.1. Then $\bar{\rho}\left(\pi_{1}(M(\alpha))\right)=\bar{T}_{12}$ (Lemma $5.3(1))$, and as $\alpha \in \operatorname{ker}(\bar{\rho})$, we have that $\bar{\rho}\left(\pi_{1}(\partial M)\right)$ is a cyclic group of order $q=1$ or 2 or 3 . Lemma 4.2 implies $q \neq 1$. Further, the kernel of $\bar{\rho}$ is cyclic by Lemma 5.4, and so the conclusion follows from Theorem 2.1.

(4) The proof is similar to that of (2).

(5) (a) The inequality (i) comes from Corollary 5.7. For part (ii), we may assume, as in (2), that $\|\alpha\|>s$. If $\|\alpha\|>s+2$, it follows from Theorem 2.2 that there are at least two points $x \in X_{0}^{\nu}$ such that $Z_{x}\left(f_{\alpha}\right)=2>Z_{x}\left(f_{\theta}\right)=0$. Now $H_{1}(M)$ has no even torsion, and so, by Lemma $5.3(2)$ and the discussion following Lemma 5.5, there is at least one point $x \in X_{0}^{\nu}$ such that an associated representation $\rho \in R_{0}$ provided by Lemma 4.1, satisfies $\bar{\rho}\left(\pi_{1}(M(\alpha))=\bar{O}_{24}\right.$. Hence $\bar{\rho}\left(\pi_{1}(\partial M)\right)$ is a cyclic group of order $q=1$ or 2 or 3 or 4 . Again, $q \neq 1$ by Lemma 4.2. Further, the kernel of $\bar{\rho}$ is cyclic by Lemma 5.4, and so the conclusion follows from Theorem 2.1.

(b) Again, the inequality (i) comes from Corollary 5.7. For (ii), we proceed as in part (a), using the hypothesis $\|\alpha\|>s+4$ to produce a representation $\rho \in R_{0}$ such that $\bar{\rho}\left(\pi_{1}(M(\alpha))\right)=\bar{O}_{24}$. Applying Lemma 5.4 and Theorem 2.1, we also obtain a sublattice $\tilde{L}$ of $L$, which has index $q=2, q=3$, or $q=4$, which contains $\alpha$, and for which $\alpha$ realizes the minimal norm amongst all its non-trivial elements. To finish the proof, we must explain why the hypothesis that $H_{1}(M)$ has even torsion implies that $q$ cannot be 4 .

Now according to Lemma 5.1, $H_{1}(M)=\mathbf{Z} \oplus \mathbf{Z}_{2 a}$ where $a$ is an odd integer, and therefore $H_{1}\left(M ; \mathbf{Z}_{2}\right)=\mathbf{Z}_{2} \oplus \mathbf{Z}_{2}$. Noting that $H_{1}\left(M(\alpha) ; \mathbf{Z}_{2}\right)=\mathbf{Z}_{2}$, we see that $\alpha$ must be non-trivial when considered as an element of $H_{1}\left(M ; \mathbf{Z}_{2}\right)$. On the other hand, Lefschetz duality shows that the kernel of the homomorhism $H_{1}\left(\partial M ; \mathbf{Z}_{2}\right) \rightarrow H_{1}\left(M ; \mathbf{Z}_{2}\right)$ is isomorphic to $\mathbf{Z}_{2}$. Thus, there is a basis $\{\alpha, \beta\}$ of $H_{1}(\partial M)$, where $\beta$ is trivial when considered as a class in $H_{1}\left(M ; \mathbf{Z}_{2}\right)$. The composition $\pi_{1}(\partial M) \rightarrow \pi_{1}(M) \rightarrow \pi_{1}(M(\alpha)) \rightarrow \bar{O}_{24} \rightarrow H_{1}\left(\bar{O}_{24}\right)=\mathbf{Z}_{2}$ obviously contains $\alpha$ in its kernel. But further, it factors through the arrow $H_{1}\left(\partial M ; \mathbf{Z}_{2}\right) \rightarrow H_{1}\left(M ; \mathbf{Z}_{2}\right)$, and so $\beta$ is also in its kernel. Thus, $\pi_{1}(\partial M)$ is sent by $\bar{\rho}$ into the kernel of $\bar{O}_{24} \rightarrow H_{1}\left(\bar{O}_{24}\right)=\mathbf{Z}_{2}$. But this kernel is isomorphic to $\bar{T}_{12}$, a group which contains no elements of order 4 . Hence, the order $q$ of $\bar{\rho}\left(\pi_{1}(\partial M)\right)$ in $\bar{\rho}\left(\pi_{1}(M)\right)=\bar{O}_{24}$ is either 2 or 3 . 


\section{THE GEOMETRY OF THE FUNDAMENTAL POLYGON}

The goal of this section is to develop a sequence of technical lemmas that will be necessary for the proof of Theorem 1.1. Henceforth in this paper, we shall use the following fixed notations and terminology: $L=H_{1}(\partial M ; \mathbf{Z}), V=H_{1}(\partial M ; \mathbf{R})$, $s=\min \{\|\delta\| ; \delta \in L, \delta \neq 0\}, B$ is the disc in $V$ of radius $s, k B$ is the disc in $V$ of radius $k s, \theta$ is a fixed class of $L$ such that $\theta \in \partial B, \tau \in L$ is a fixed class such that $\theta$ and $\tau$ form a basis of $L$. Those elements in $L$ whose $\tau$-coordinate is \pm 1 will be referred to as integral elements. Besides a vector space with the norm $\|\cdot\|, V$ is also considered as a standard $(x, y)$-plane with Euclidean metric, in which $L$ becomes the set of integer lattice points through the identifications $\theta=(1,0), \tau=(0,1)$. By a pair of elements in $V$, we mean $(a, b)$ and $(-a,-b)$. By a slope of $L$, we mean a pair of primitive elements of $L$. Classes in $L$ of the form $(m, 1)$ will be called integral classes.

Recall that $k B$ is a compact, convex, finite-sided polygon in $V$ which is balanced (i.e. $-(k B)=k B)$. Since $\operatorname{int}(B) \cap L=\{(0,0)\}$, the Euclidean area of $B$ is no larger than 4 ([CGLS, page 244]).

The proof of Theorem 1.1 is based on the inequalities which were obtained in Theorem 2.3. When $s$ is large, at least 10 say, the proof depends only on these inequalities and the general properties of $B$. On the other hand, the arguments needed for the cases where $s$ is small are much more delicate, depending on a fine analysis of the geometry of $B$. It is this analysis which occupies most of this section and the next.

We say that a strict boundary slope $\pm \beta$ and an ideal point $x$ of $\tilde{X}_{0}$ are associated if $\pm \beta$ is the unique slope such that $\Pi_{x}\left(f_{\beta}\right)=0$. We note that it is possible for a given slope to be associated to several ideal points, and it is also possible for an ideal point $x$ to have no associated slopes, that is $\Pi_{x}\left(f_{\delta}\right)=0$ for each class $\delta \in L$.

Lemma 6.1. The pairs of vertices of the fundamental polygon $B$ correspond precisely to the distinct strict boundary slopes of $M$ associated to some ideal point of $\tilde{X}_{0}$.

Proof. It is shown in $\S 1.4$ of [CGLS] that each pair of vertices of $B$ corresponds to a strict boundary slope of $M$ associated to some ideal point of $\tilde{X}_{0}$, and so we must show that all such boundary slopes arise in this way.

Let $x_{1}, x_{2}, \ldots, x_{r}$ be the ideal points of $\tilde{X}_{0}$ associated to some strict boundary slope of $M$. In [CGLS, \$1.4], it is shown that for each $i=1,2, \ldots, r$, there exists a non-trivial real linear function $\psi_{i}: V \rightarrow \mathbf{R}$ such that for each $\delta \in L$, we have $\Pi_{x}\left(f_{\delta}\right)=\left|\psi_{i}(\delta)\right|$. Note that the kernel of $\psi_{i}$ is the linear subspace of $V$ spanned by the boundary class associated to $x_{i}$. It follows that if $\pm \beta_{1}, \pm \beta_{2}, \ldots, \pm \beta_{t}$ are the strict boundary slopes associated to some ideal point of $\tilde{X}_{0}$, then there are non-trivial real linear functions $\phi_{j}: V \rightarrow \mathbf{R}, 1 \leq j \leq t$, such that for each $j, \beta_{j}$ spans the kernel of $\phi_{j}$, and such that for each $\delta \in L$, we have $\|\delta\|=\left|\phi_{1}(\delta)\right|+\left|\phi_{2}(\delta)\right|+\ldots+\left|\phi_{t}(\delta)\right|$. Owing to the fact that the slopes $\pm \beta_{1}, \pm \beta_{2}, \ldots, \pm \beta_{t}$ are distinct, there is a neighbourhood of $v_{1}=\frac{s}{\left\|\beta_{1}\right\|} \beta_{1}$ such that for each point $(x, y)$ in this neighbourhood, we have $\|(x, y)\|=\left|\phi_{1}(x, y)\right|+\rho(x, y), \rho: V \rightarrow \mathbf{R}$ being linear and non-trivial. Now $v_{1}$ is a vertex of $B$ if and only if the two lines $\phi_{1}(x, y)+\rho(x, y)=s$ and $-\phi_{1}(x, y)+\rho(x, y)=$ $s$ intersect transversely at $v_{1}$. This intersection is either a line or a point, and as $s \neq 0$, it does not contain $(0,0)$. But then, it cannot be a line as it is contained in the kernel of $\phi_{1}$, i.e. in the linear subspace determined by $\beta_{1}$. Thus $v_{1}$ is indeed a 
vertex of $B$, and in a similar fashion, we may show that $v_{j}=\frac{s}{\left\|\beta_{j}\right\|} \beta_{j}$ is a vertex of $B$ for each $j=2,3, \ldots, t$. This completes the proof of Lemma 6.1.

Denote by $x_{1}, x_{2}, \ldots, x_{r}$ the ideal points of $\tilde{X}_{0}$ associated to some strict boundary slope other than the slope $\pm \theta$, and by $y_{1}, y_{2}, \ldots, y_{t}$ the ideal points associated to $\pm \theta$. Let $\pm \beta_{i}$ be the slope associated to $x_{i}, 1 \leq i \leq r$, and note that there may be repetitions amongst the classes $\beta_{1}, \beta_{2}, \ldots, \bar{\beta}_{r}$. The next lemma will be used frequently in this section.

Lemma 6.2. Let $x \in \tilde{X}_{0}$ be an ideal point having an associated strict boundary class $\beta=(m, n) \in L$. Then $|n|=\Delta(\theta, \beta)$, and

(1) if $n \neq 0$, then $n \mid \Pi_{x}\left(I_{\theta}\right)$ and for any $\delta \in L, \Pi_{x}\left(I_{\delta}\right)=\frac{\Delta(\delta, \beta)}{\Delta(\theta, \beta)} \Pi_{x}\left(I_{\theta}\right)$;

(2) if $n=0$, then for any $\delta \in L, \Pi_{x}\left(I_{\delta}\right)=\Delta(\delta, \theta) \Pi_{x}\left(I_{\tau}\right)$;

(3) for any class $\delta \in L$,

$$
\|\delta\|=2 \sum_{1 \leq i \leq r} \frac{\Delta\left(\delta, \beta_{i}\right)}{\Delta\left(\theta, \beta_{i}\right)} \Pi_{x_{i}}\left(I_{\theta}\right)+2 \Delta(\delta, \theta) \sum_{1 \leq j \leq t} \Pi_{y_{j}}\left(I_{\tau}\right) .
$$

Proof. That $|n|=\Delta(\theta, \beta)$ is clear.

(1) Write $\delta=(p, q)$ and recall from the proof of [CGLS, 1.4.1] that there is a degree-two extension field $E$ of the function field $F=\mathbf{C}\left(R_{0}\right)$ such that the tautological representation $P: \pi_{1}(M) \rightarrow S L_{2}(F)$ is conjugate in $S L_{2}(E)$ to a representation $P^{\prime}: \pi_{1}(M) \rightarrow S L_{2}(E)$ which restricts to a diagonal representation of $\pi_{1}(\partial M)$, and the valuation $w: K^{*} \rightarrow \mathbf{Z}$ associated to the ideal point $x$ can be extended to a valuation $v: E^{*} \rightarrow \mathbf{Z}$ such that $\left.v\right|_{K^{*}}=d w$ for some positive integer d. Moreover, if we let $P^{\prime}(\theta)=\left(\begin{array}{cc}a & 0 \\ 0 & a^{-1}\end{array}\right)$ and $P^{\prime}(\tau)=\left(\begin{array}{cc}b & 0 \\ 0 & b^{-1}\end{array}\right), a, b \in E^{*}$, then for any $\delta=(p, q) \in L, \Pi_{x}\left(I_{\delta}\right)=\frac{1}{d}|p v(a)+q v(b)|$. Let $\Pi_{x}\left(I_{\theta}\right)=j$. Since $x$ is one of $x_{1}, x_{2}, \ldots, x_{r}, j \neq 0$. Then $v(a)=\epsilon d j$ for some $\epsilon= \pm 1$. Let $\Pi_{x}\left(I_{\tau}\right)=k$ for some integer $k \geq 0$. Then $v(b)=\sigma d k$ for some $\sigma= \pm 1$. By our assumption, $\Pi_{x}\left(I_{\beta}\right)=\frac{1}{d}|m v(a)+n v(b)|=0$, i.e. $m \epsilon j+n \sigma k=0$. Thus $n \mid j$. Also for any $\delta=(p, q) \in L, \Pi_{x}\left(I_{\delta}\right)=|\epsilon p j+\sigma q k|=|\epsilon n p j+\sigma n q k| /|n|=$ $|\epsilon(n p-m q) j+(\epsilon m j+\sigma n k) q| /|n|=|n p-m q| j /|n|$. This gives (1).

The proof of (2) is similar, while that of (3) follows directly from (1), (2) plus the identity

$$
\|\delta\|=2 \sum_{\text {ideal } x \in \tilde{X}_{0}} \Pi_{x}\left(I_{\delta}\right)
$$

An immediate consequence of Lemma 6.2 is

Lemma 6.3. (1) For any non-zero element $\delta \in L,\|\delta\|$ is a positive even integer.

(2) Suppose that $B$ has $k$ pairs of vertices. Then $s \geq 2 k-2$, and if $\partial B$ contains a lattice point which is not a vertex of $B$, then $s \geq 2 k$.

Lemma 6.4. For any $(a, b) \in B,|b| \leq 2$. If there is $(a, b) \in B$ with $b=2$, then $B$ is a parallelogram with vertices $\pm(1,0)$ and $\pm(a, b)$, and further, $(a, b) \in L$.

Proof. Suppose that there is a point $(a, b) \in B$ with $|b| \geq 2$. Let $P$ be the parallelogram in $V$ with vertices $\pm(a, b)$ and $\pm(1,0)$. Then $P \subset B$ and $\operatorname{Area}(P)=2|b| \geq 4$. On the other hand $\operatorname{Area}(P) \leq \operatorname{Area}(B) \leq 4$. Hence $\operatorname{Area}(B)=\operatorname{Area}(P)=4$, and 
we conclude that $P=B$ and $b=2$. Finally, observe that $a$ must then be an integer as otherwise, $B$ would contain a non-trivial integral element of $L$ in its interior.

Lemma 6.5. Suppose that $\theta$ is not a vertex of $B$. Then $s \geq 4$. Moreover,

(1) if $s=4$, then $B$ is a parallelogram with vertices $\pm(a, 2 /(k+2))$ and $\pm(a+2,2 /(k+2))$, for some integer $k \geq 0$ such that $a(k+2) / 2 \in \mathbf{Z}$;

(2) if $s=6$, then either $B$ is a parallelogram with vertices $\pm(3(2 m+1) /|k|, 6 /|k|)$ and $\pm(3(2 m+1+k) / 2|k|, 3 /|k|)$, for some integer $m$ and odd integer $k$ with $|k| \geq 5$, or $B$ is a parallelogram with vertices $\pm(3 m /|k|, 3 /|k|)$ and $\pm(3(m+k) / 2|k|$, $3 / 2|k|)$, for some integers $m$ and $k$ with $|k| \geq 3$, or $B$ has three pairs of vertices $\pm(3 m /(2 j+q), 3 /(2 j+q)), \pm(3(m+j) /(j+q), 3 /(j+q))$ and $\pm(3(m+j+q) /$ $(j+2 q), 3 /(j+2 q))$, for some integers $m, j>0, q>0$ with $j+q \geq 3$;

(3) if $s=8$, then (i) $B$ has at most four pairs of vertices whose associated strict boundary classes have $\tau$-coordinate no larger than 3 in absolute value. Moreover, if $B$ has one pair of vertices whose associated boundary slope has $\tau$-coordinate equal to 3 in absolute value, then there is at most one other vertex, and its associated boundary slope has $\tau$-coordinate equal to 1 in absolute value. (ii) If $B$ has at least two pairs of vertices with their associated strict boundary slopes being non-integral, then $B$ is a parallelogram, the two associated strict boundary slopes have their $\tau$ coordinate equal to 2 in absolute value.

Proof. The inequality $s \geq 4$ is a consequence of Lemma 6.3.

(1) By Lemma $6.3(2), B$ has exactly two pairs of vertices $\pm\left(a_{i}, b_{i}\right), i=1,2$, and thus $B$ is a parallelogram. Our hypotheses imply that $I_{\theta}$ has a pole at each ideal point associated to a vertex of $B$. Thus, from the identity $\|\theta\|=s=4$, we conclude that $I_{\theta}$ has exactly two poles $x_{i}, i=1,2$, each of order 1 , i.e. $\Pi_{x_{i}}\left(I_{\theta}\right)=1$. Hence, the associated strict boundary classes $\beta_{i}$ are integral classes by Lemma 6.2 (1). Now Lemma $6.2(3)$ shows that $\left\|\beta_{1}\right\|=\left\|\beta_{2}\right\|=s+2 k=(k+2) s / 2$, for some integer $k \geq 0$. Thus $\left|b_{1}\right|=\left|b_{2}\right|=2 /(k+2)$, and $a_{2}=a_{1}+2$ or $a_{2}=a_{1}-2$, since $\theta \in \partial B$.

(2) If $s=6$, then $B$ has either two or three pairs of vertices (Lemma 6.3).

Consider first the case where $B$ has two (pairs of) vertices $\Lambda_{i}, i=1,2$, so that $B$ is a parallelogram. Let $\pm \beta_{i} \in L$ be the strict boundary slope which is a rational multiple of $\Lambda_{i}$ and let $\mathcal{I}_{i} 1 \tilde{X}_{0}$ be the set of ideal points associated to $\beta_{i}$. Now $\|\theta\|=6$ and therefore by Lemma 6.1 and Lemma 6.2 (3) we may assume that $\sum_{x \in \mathcal{I}_{1}} \Pi_{x}\left(I_{\theta}\right)=2$ and $\sum_{x \in \mathcal{I}_{2}} \Pi_{x}\left(I_{\theta}\right)=1$. Hence Lemma $6.2(1)$ implies that either $\beta_{1}=(m, 1)$ and $\beta_{2}=(p, 1)$, or $\beta_{1}=(2 m+1,2)$ and $\beta_{2}=(p, 1)$.

If $\beta_{1}=(2 m+1,2)$ and $\beta_{2}=(p, 1)$, then $\left\|\beta_{1}\right\|=\left\|\beta_{2}\right\|=2|2 p-2 m-1|$ by Lemma $6.2(3)$. Setting $k=2 p-2 m-1$, we see that $k$ is an odd integer satisfying $\left\|\beta_{1}\right\|=\left\|\beta_{2}\right\|=|k| s / 3$. From $\left(a_{i}, b_{i}\right)=\frac{3}{|k|} \beta_{i}$ and Lemma 6.4, we obtain $b_{i}=\frac{6}{|k|}<2$, so that $|k| \geq 5$. Evidently,

$$
\Lambda_{1}=(3(2 m+1) /|k|, 6 /|k|) \quad \text { and } \quad \Lambda_{2}=(3(2 m+1+k) / 2|k|, 3 /|k|) .
$$

If $\beta_{1}=(m, 1)$ and $\beta_{2}=(p, 1)$, then $\left\|\beta_{2}\right\|=2\left\|\beta_{1}\right\|=4|p-m|$ by Lemma $6.2(3)$. Setting $k=p-m$, we have $\left\|\beta_{2}\right\|=2|k| s / 3,\left\|\beta_{1}\right\|=|k| s / 3$ and further, $|k| \geq 3$ as $\left\|\beta_{1}\right\| \geq 6$. Evidently, $\Lambda_{1}=(3 m /|k|, 3 /|k|)$ and $\Lambda_{2}=(3(m+k) / 2|k|, 3 / 2|k|)$.

Next consider the case where $B$ has three (pairs of) vertices $\Lambda_{i}, i=1,2,3$. Let $\beta_{i} \in L$ be the associated strict boundary class, and $x_{i} \in \tilde{X}_{0}$ an associated pole of $I_{\theta}$. Then $\Pi_{x_{i}}\left(I_{\theta}\right)=1$, and $\beta_{i}$ is an integral slope, for $i=1,2,3$. We may assume that $\beta_{1}=(m, 1), \beta_{2}=(m+j, 1)$ and $\beta_{3}=(m+j+q, 1)$, for some integers $m, j>0$, 
$q>0$. Then $\left\|\beta_{1}\right\|=2(2 j+q)=(2 j+q) s / 3,\left\|\beta_{2}\right\|=2(j+q)=(j+q) s / 3$, and $\left\|\beta_{3}\right\|=2(j+2 q)=(j+2 q) s / 3$. Thus, from the inequality $\left\|\beta_{2}\right\| \geq 6$, we see that $(j+q) \geq 3$. Finally, $\Lambda_{1}=(3 m /(2 j+q), 3 /(2 j+q)), \Lambda_{2}=(3(m+j) /(j+q), 3 /(j+q))$, and $\Lambda_{3}=(3(m+j+q) /(j+2 q), 3 /(j+2 q))$.

(3) This follows from Lemmas 6.2 and 6.3 .

Lemma 6.6. Suppose that all points of $\partial B \cap L$ are vertices of $B$.

(1) If $s=2$, then $B$ is a parallelogram with vertices $\pm(1,0)$ and $\pm\left(\frac{m}{k}, \frac{1}{k}\right)$, for some integer $m$ and some integer $k \geq 1$.

(2) If $s=4$, then either (i) $B$ is a parallelogram with vertices $\pm(1,0)$ and $\pm\left(\frac{2 m}{k+2}, \frac{2}{2+k}\right)$, for some integers $m$ and $k \geq 0$, or (ii) $B$ is a parallelogram with vertices $\pm(1,0)$ and $\pm\left(\frac{2(2 j+1)}{2+k}, \frac{4}{2+k}\right)$, for some integers $j$ and $k \geq 2$, or (iii) $B$ is a polygon with vertices $\pm(1,0), \pm\left(\frac{2 m}{k+2}, \frac{2}{k+2}\right)$ and $\pm\left(\frac{2(m+j)}{k+2}, \frac{2}{k+2}\right)$, for some integers $m, j, k$ with $j \geq 1$ and $k \geq j-1$.

(3) If $s=6$, then either (i) $B$ is contained in a parallelogram with vertices $\pm(1,0)$ and $\pm(m, 1)$, with $(m, 1)$ being a strict boundary class associated to a vertex of $B$; or (ii) $B$ is contained in a parallelogram with vertices $\pm(1,0)$ and $\left(\frac{6 m}{5}, \frac{6}{5}\right)$, with $(m, 2)$ being a strict boundary class associated to a vertex of $B$; or (iii) $B$ is contained in a parallelogram with vertices $\pm(1,0)$ and $(a, 1)$, with $(3 a, 3)$ being a strict boundary class associated to a vertex of $B$; or (iv) $B$ is contained in the polygon with vertices $\pm(1,0), \pm(m, 1), \pm\left(\frac{3(2 m+1)}{5}, \frac{6}{5}\right)$, or the polygon with vertices $\pm(1,0), \pm(m+1,1), \pm\left(\frac{3(2 m+1)}{5}, \frac{6}{5}\right)$, with $(2 m+1,2)$ and $(m, 1)$ or $(2 m+1,2)$ and $(m+1,1)$ being strict boundary classes associated to vertices of $B$; or $(\mathrm{v})$ $B$ is a polygon with vertices $\pm(1,0),\left(\frac{3 m}{|k|+q}, \frac{3}{|k|+q}\right)$ and $\left(\frac{3(m-k)}{2|k|+q}, \frac{3}{2|k|+q}\right)$, for some integers $m, k \neq 0, q>0$, satisfying $|k|+q \geq 3$, or (vi) $B$ is a polygon with vertices $\pm(1,0), \pm(3 m /(2 j+k+q), 3 /(2 j+k+q)), \pm(3(m+j) /(j+k+q), 3 /(j+k+q))$ and $\pm(3(m+j+k) /(j+2 k+q), 3 /(j+2 k+q))$, for some integers $m, j>0, k>0, q>0$.

Proof. The arguments here are similar to those employed in the proof of Lemma 6.5, so that some details will be omitted.

(1) By Lemma $6.3(2), B$ has exactly two pairs of vertices, $\pm \Lambda_{i}= \pm\left(a_{i}, b_{i}\right)$, $i=1,2$. Let $x_{i} \in \tilde{X}_{0}$ and $\pm \beta_{i} \in L$ be respectively an ideal point and the strict boundary slope, associated to $\pm \Lambda_{i}$. We may assume that $\theta=\beta_{1}$. Then, $\Pi_{x_{1}}\left(I_{\theta}\right)=0$ and $\Pi_{x_{2}}\left(I_{\theta}\right)=1$. Hence by Lemma $6.2(1), \beta_{2}$ is an integral class, say $\beta_{2}=(m, 1)$ for some integer $m$. Then, $B$ is a parallelogram with vertices $\pm \theta= \pm(1,0)$ and $\pm \Lambda_{2}= \pm\left(a_{2}, b_{2}\right)$, with $\left|b_{2}\right| \leq 1$; we may assume that $0<b_{2} \leq 1$. Now Lemma 6.2 (3) shows that $\left\|\beta_{2}\right\|=\|(m, 1)\|=2 k=k s$, for some $k \geq 1$. Clearly then, $\left(a_{2}, b_{2}\right)=$ $(m / k, 1 / k)$.

(2) When $s=4$, Lemma 6.3 shows that $B$ has either two or three pairs of vertices.

In the former case, $B$ is a parallelogram contained in a parallelogram with vertices $\pm(m, n)$ and $\pm(1,0)$, for some $n=1$ or 2 (Lemma 6.2$)$. Here, $(m, n)$ is the other associated strict boundary class. If $n=1$, then we have (i). If $n=2$, then let $m=2 j+1$, and note that $(j, 1),(j+1,1)$ and $(m, 2)$ are not contained in $B$, since otherwise $\partial B \cap L$ would contain points which are not vertices of $B$. Thus $\|(j, 1)\| \geq 6,\|(j+1,1)\| \geq 6$ and $\|(m, 2)\| \geq 6$. If $\|(m, 2)\|=6$, then by studying the resulting geometry of $B$, we conclude that $\|(j+1,1)\|=5$, which is impossible by Lemma $6.3(1)$. Thus $\|(m, 2)\| \geq 8$, and we have case (ii). 
In the latter case, the other two pairs of associated strict boundary classes of $B$ are integral classes, say $(m, 1)$ and $(m+j, 1), j \geq 1$, and $\|(m, 1)\|=\|(m+j, 1)\|$, by Lemma 6.2. Then $B$ is a polygon with vertices $\pm(1,0), \pm\left(\frac{2 m}{k+2}, \frac{2}{k+2}\right), \pm\left(\frac{2(m+j)}{k+2}, \frac{2}{k+2}\right)$, for some integers $k \geq 0$ and $j \geq 1$. Now, any horizontal line in $V$ intersects $B$ in a segment of length at most 2 , and hence $k \geq j-1$, i.e. we have (iii).

(3) $B$ has either two, or three, or four pairs of vertices by Lemma 6.3 .

Suppose that $B$ has two (pairs of) vertices. Then by Lemma $6.2, B$ is a parallelogram contained in a parallelogram with vertices $\pm(m, n)$ and $\pm(1,0)$, for some $n=1,2$ or 3 , where $(m, n)$ is the other associated strict boundary class. If $n=1$, then we have (i). If $n=2$, then $m=2 j+1$. Now, neither $(j, 1)$ nor $(j+1,1)$ is contained in $B$, and so $\|(j, 1)\| \geq 8$ and $\|(j+1,1)\| \geq 8$, by Lemma 6.3 (1). It follows that $B$ is contained in a parallelogram with vertices $\pm(1,0)$ and $\left(\frac{6 m}{5}, \frac{6}{5}\right)$, i.e. we have (ii). If $n=3$, then $m=3 j+1$ or $3 j+2$. In the former case, we use the inequality $\|(j, 1)\| \geq 8$ to deduce that $\|(3 j / 4,3 / 4)\| \geq 6$. It follows that $B$ is contained in a parallelogram with vertices $\pm(1,0)$ and $((3 j+1) / 3,1)$. An analogous argument shows that in the latter case, $B$ is contained in a parallelogram with vertices $\pm(1,0)$ and $((3 j+2) / 3,1)$. This is case (iii).

Suppose that besides $\pm(1,0), B$ has two other pairs of vertices $\pm \Lambda_{i}, i=1,2$. Let $\pm \beta_{i} \in L$ be the strict boundary slope which is a rational multiple of $\Lambda_{i}$ and let $\mathcal{I}_{i} \tilde{X}_{0}$ be the set of ideal points associated to $\beta_{i}$. By Lemma 6.2 (3) we may assume that $\sum_{x \in \mathcal{I}_{1}} \Pi_{x}\left(I_{\theta}\right)=2$ and $\sum_{x \in \mathcal{I}_{2}} \Pi_{x}\left(I_{\theta}\right)=1$. Then by Lemma $6.2(1)$, we have either $\beta_{1}=(2 m+1,2)$ and $\beta_{2}=(p, 1)$, or $\beta_{1}=(m, 1)$ and $\beta_{2}=(p, 1)$. Let $\mathcal{I}_{\theta} \tilde{X}_{0}$ be the set of ideal points associated to $\theta$.

If $\beta_{1}=(2 m+1,2)$ and $\beta_{2}=(p, 1)$, then $\left\|\beta_{1}\right\|=2|2 p-2 m-1|+4 \sum_{y \in \mathcal{I}_{\theta}} \Pi_{y}\left(I_{\tau}\right)$, $\left\|\beta_{2}\right\|=2|2 p-2 m-1|+2 \sum_{y \in \mathcal{I}_{\theta}} \Pi_{y}\left(I_{\tau}\right)$, by Lemma $6.2(3)$. If $p>m+1$, then Lemma $6.2(3)$ shows that $\|(m+1,1)\|=\|(p, 1)\|$, and thus our hypotheses on $B$ now force the identity $\|(m+1 / 2,1)\|=\|(p, 1)\|$. Hence $\left\|\beta_{1}\right\|=2\left\|\beta_{2}\right\|$, which contradicts our previous calculations. Thus $p \leq m+1$, and a similar argument shows that $p \geq m$. In the case $p=m$, notice that $(m+1,1)$ is not in $B$, as otherwise $(2 m+1,2)$ would be a vertex of $B$, contradicting Lemma 6.4. Thus $\|(m+1,1)\| \geq 8$, and so $\left\|\left(\frac{3(m+1)}{4}, \frac{3}{4}\right)\right\| \geq 6=s$. Consideration of the line through $\theta$ and $\left(\frac{3(m+1)}{4}, \frac{3}{4}\right)$ shows that $B$ is contained in the polygon with vertices $\pm(1,0)$, $\pm(m, 1), \pm\left(\frac{3(2 m+1)}{5}, \frac{6}{5}\right)$. Similarly, if $p=m+1, B$ is contained in the polygon with vertices $\pm(1,0), \pm(m+1,1), \pm\left(\frac{3(2 m+1)}{5}, \frac{6}{5}\right)$, i.e. we have (iv).

If $\beta_{1}=(m, 1)$ and $\beta_{2}=(p, 1)$, then $\left\|\beta_{1}\right\|=2|m-p|+2 \sum_{y \in \mathcal{I}_{\theta}} \Pi_{y}\left(I_{\tau}\right),\left\|\beta_{2}\right\|=$ $4|m-p|+2 \sum_{y \in \mathcal{I}_{\theta}} \Pi_{y}\left(I_{\tau}\right)$. Set $k=m-p$ and $q=\sum_{y \in \mathcal{I}_{\theta}} \Pi_{y}\left(I_{\tau}\right)$. Then $\left\|\beta_{1}\right\|=$ $2|k|+2 q=(|k|+q) s / 3$ and $\left\|\beta_{2}\right\|=4|k|+2 q=(2|k|+q) s / 3$. Thus $B$ is a polygon with vertices $\pm(1,0),\left(\frac{3 m}{|k|+q}, \frac{3}{|k|+q}\right)$ and $\left(\frac{3(m-k)}{2|k|+q}, \frac{3}{2|k|+q}\right)$, where $|k|+q \geq 3$. So we have $(\mathrm{v})$.

Finally, suppose that besides $\pm(1,0), B$ has three other pairs of vertices $\pm \Lambda_{i}$, $i=1,2,3$. For each value of $i$, let $\pm \beta_{i} \in L$ be the strict boundary slope which is a rational multiple of $\Lambda_{i}$. As $\|\theta\|=6$, Lemma 6.2 (3) implies that for each $i$ there is a unique ideal point $x_{i}$ associated to $\beta_{i}$ and further that $\Pi_{x_{i}}\left(I_{\theta}\right)=1$. Thus Lemma 6.2 (1) shows that $\pm \beta_{i}$ is an integral slope (i.e. has $\tau$-coordinate equal to \pm 1$)$, for $i=1,2,3$. We may assume that $\beta_{1}=(m, 1), \beta_{2}=(m+j, 1)$ and $\beta_{3}=(m+j+k, 1)$, for some integers $m, j>0, k>0$. Then letting $q$ be the sum over the ideal points $y$ associated to $\theta$ of the multiplicities $\Pi_{y}\left(I_{\tau}\right)$, we have $q>0$, 
$\left\|\beta_{1}\right\|=(2 j+k+q) s / 3,\left\|\beta_{2}\right\|=(j+k+q) s / 3$, and $\left\|\beta_{3}\right\|=(j+2 k+q) s / 3$. It is evident that $\Lambda_{1}=(3 m /(2 j+k+q), 3 /(2 j+k+q)), \Lambda_{2}=(3(m+j) /(j+k+q), 3 /(j+k+q))$ and $\Lambda_{3}=(3(m+j+k) /(j+2 k+q), 3 /(j+2 k+q))$. This is case (vi).

The final lemma of this section will be used to analyse those cases where $M$ admits slopes which are both boundary slopes and finite/cyclic slopes.

Lemma 6.7. If $\alpha \in L$ is a strict boundary class which is also a finite/cyclic surgery class, then $\Delta(\alpha, \beta) \leq 1$ for any surgery class $\beta \in L$ such that $M(\beta)$ either has finite/cyclic fundamental group or is an irreducible non-Haken manifold.

Proof. If $M$ has first Betti number equal to 1, then by [CGLS, Theorem 2.0.3 and Addendum 2.0.4], $M$ contains a closed incompressible surface $S$ which remains incompressible in $M(\delta)$, for any primitive class $\delta \in L$ satisfying $\Delta(\alpha, \delta)>1$. The hypotheses on $M(\beta)$ imply that $S$ must be compressible in $M(\beta)$, and thus $\Delta(\alpha, \beta) \leq 1$. If $M$ has first Betti number larger than 1 , then the hypotheses on $M(\beta)$ imply that both $\alpha$ and $\beta$ are necessarily infinite cyclic surgery slopes (cf. Lemma 5.1), and thus the conclusion now follows from the cyclic surgery theorem.

\section{Proof OF TheOREM 1.1}

Throughout this section we shall continue to use the notation developed in $\S 6$, and we shall also assume that all the hypotheses of Theorem 1.1 hold. Theorem 1.1 will follow from Lemma 7.1, Proposition 7.2 and Lemma 7.3 given below.

Lemma 7.1. Suppose that there are at least two slopes on $\partial M$ which are at the same time strict boundary slopes and finite/cyclic slopes. Then, there are no more than four slopes on $\partial M$ which are finite/cyclic slopes, and the distance between any two such slopes is at most 2.

Proof. Suppose that $\pm \alpha, \pm \beta \in L$ are distinct slopes which are at the same time strict boundary slopes and finite/cyclic slopes. Lemma 6.7 shows that $\Delta(\alpha, \delta) \leq 1$, $\Delta(\beta, \delta) \leq 1$, and $\Delta(\alpha, \beta)=1$, for any finite/cyclic surgery class $\delta \in L$. It is easy to verify that $\delta \in\{ \pm \alpha, \pm \beta, \pm(\alpha+\beta), \pm(\alpha-\beta)\}$. Thus, there are at most four surgery slopes which are finite/cyclic, and their mutual distance is at most 2 .

Proposition 7.2. Let \#, $\Delta, n_{0}$ and $m_{0}$ denote respectively the number, the maximal mutual distance, the maximal absolute value of the $\tau$-coordinates and the number of integral slopes of all finite/cyclic surgery slopes which are not strict boundary slopes on $\partial M$. Then $\# \leq 6, \Delta \leq 5, n_{0} \leq 2$ and $m_{0} \leq 5$.

Assuming Proposition 7.2 holds, we can show the following.

Lemma 7.3. Suppose that there is only one slope on $\partial M$ which is at the same time a strict boundary slope and finite/cyclic slope. Then there are at most six finite/cyclic slopes and their mutual distance is at most 5.

Proof. Let $\pm \alpha$ be the slope which is at the same time a strict boundary slope and a finite/cyclic slope. If $\pm \alpha$ is the slope $\pm \theta$, then by Lemma 6.7, all other finite/cyclic slopes of $L$ are distance one from $\pm \alpha$ and thus are integral slopes. By Proposition 7.2 , the number of such integral boundary slopes, $m_{0}$, is at most 5 and their maximal mutual distance is at most 5. Hence Lemma 7.3 holds when $\pm \alpha= \pm \theta$. 
Now suppose that $\pm \alpha \neq \pm \theta$. Then by Proposition 7.2, all other finite/cyclic slopes of $L$ have mutual distance at most 5 and have $\tau$-coordinates at most two, and further all these slopes, by Lemma 6.7, are distance one from $\pm \alpha$. Now it is easy to verify that there are at most five slopes which can have $\tau$ coordinates at most two and are also distance one from a fixed slope that is not the slope $\pm \theta$. The lemma follows.

Proof of Theorem 1.1. Note that Theorem 1.1 (1) is deducible directly from Lemma 7.1, Proposition 7.2 and Lemma 7.3. To deduce Theorem 1.1 (2), let $\alpha$ be a cyclic class and $\beta$ a finite/cyclic class in $L$. If $\alpha$ or $\beta$ is also a strict boundary class, then Lemma 6.7 shows that $\Delta(\alpha, \beta) \leq 1$. If neither $\alpha$ nor $\beta$ is such a boundary class, then $\|\alpha\|=s$ ([CGLS, Corollary 1.1.4]). Thus, we may take $\theta=\alpha$ and apply Proposition 7.2 to deduce that $\Delta(\alpha, \beta) \leq n_{0} \leq 2$. Thus Theorem 1.1 (2) holds.

The rest of this section is devoted to the proof of Proposition 7.2. We shall prove the proposition by dividing it into the following two main claims.

Main Claim I. Suppose that $\theta$ is not a vertex of $B$. Then $\# \leq 6, \Delta \leq 5, n_{0} \leq 2$ and $m_{0} \leq 5$.

Main Claim II. Suppose that all points of $\partial B \cap L$ are vertices of $B$. Then $\# \leq 6$, $\Delta \leq 5, n_{0} \leq 2$ and $m_{0} \leq 5$

The proofs of the two main claims split into many cases, parameterized by the minimal norm $s$ and the possible shapes of the fundamental polygon $B$. In each case we determine all the primitive classes in $L$ which can possibly be finite/cyclic classes, but which are not strict boundary classes, mainly using Theorem 2.3 and the results from $\S 6$. Counting these classes, and noting their relative positions, give the two main claims. Throughout the proofs of the two main claims, whenever a finite/cyclic slope is mentioned, it is assumed not to be a strict boundary slope.

Proof of Main Claim I. First note that in the proof of Main Claim I, we shall always count $\pm \theta$ as a (possible) finite/cyclic slope. Thus $\# \leq 6$ will imply $m_{0} \leq 5$ and so we shall not have to count $m_{0}$ in Main Claim I. Note also that by Lemma 6.5 , $s \geq 4$. We shall consider the three cases $s=4, s=6$, and $s \geq 8$ separately.

Case I. 1. $s=4$. Then for any finite/cyclic surgery classes $\alpha,\|\alpha\| \leq 4 s$, i.e. $\alpha \in 4 B$, by Theorem 2.3. Lemma 6.5 (1) implies that $B$ is a parallelogram with vertices $\pm(a, 2 /(k+2))$ and $\pm(a+2,2 /(k+2))$, for some integer $k \geq 0$.

Suppose $k=0$. Then, we may assume that $a=m-1$, for some integer $m$, implying that $4 B$ is a parallelogram with vertices $\pm(4 m-4,4)$ and $\pm(4 m+4,4)$. Elements in $4 B \cap L$ are $(j m+i, j)$, where $i, j=0, \pm 1, \pm 2, \pm 3, \pm 4$.

Claim. $\delta=(3 m+1,3)$ is not a finite/cyclic surgery class.

Proof of the claim. Suppose that $\delta$ is a finite/cyclic surgery class. Then, as $\|\delta\|=$ $3 s, \delta$ is an $I$-type class or an $O$-type class (Theorem 2.3 ).

If $\delta$ is an $O$-type class, then by Theorem $2.3(5),\|\delta\| \leq\left\|\delta \beta^{q}\right\|$ for any $\beta \in L$, where $q=2$ or 3 . But then $3 s=\|\delta\|=\left\|\theta^{3 m+1} \tau^{3}\right\| \leq\|\theta\|=s$ if $q=3$, or $3 s=\|\delta\|=\left\|\theta^{3 m+1} \tau^{3}\right\| \leq\left\|\theta^{m+1} \tau\right\|=s$ if $q=2$, which gives a contradiction.

If $\delta$ is an $I$-type class, then by Theorem $2.3(4),\|\delta\| \leq\left\|\delta \beta^{q}\right\|$ for any $\beta \in L$, where $q=2$ or 3 or 5 . If $q=5$, then $3 s=\|\delta\|=\left\|\theta^{3 m+1} \tau^{3}\right\| \leq\left\|\theta^{-2 m+1} \tau^{-2}\right\|=2 s$, which is again absurd. Similarly, $q \neq 3$ and $q \neq 2$. The claim is proved. 
Using related arguments, one can show that none of the classes $(4 m-3,4)$, $(4 m-1,4),(4 m+1,4),(4 m+3,4),(3 m-4,3),(3 m-2,3),(3 m-1,3),(3 m+2,3)$, $(3 m+4,3),(2 m+3,2),(2 m-3,2),(m+4,1),(m+3,1)(m-3,1)$ and $(m-4,1)$ can be a finite/cyclic surgery class. Hence, the only primitive classes in $4 B$ which can possibly be finite/cyclic surgery classes are $\pm(2 m-1,2), \pm(2 m+1,2), \pm(m-2,1)$, $\pm(m-1,1), \pm(m, 1), \pm(m+1,1), \pm(m+2,1)$ and $\pm(1,0)$. Noticing that $\pm(m-1,1)$ and $\pm(m+1,1)$ are strict boundary slopes, we obtain $\# \leq 6, \Delta \leq 5$ and $n_{0} \leq 2$.

A similar analysis proves that for $k \geq 1$, Main Claim I holds. Note that when $k \geq 7,4 B \cap L$ does not contain any non-trivial elements other than $\pm \theta$.

Case I.2. $s=6$. Then for any finite/cyclic surgery class $\alpha,\|\alpha\| \leq 3 s$, i.e. $\alpha \in 3 B$, by Theorem 2.3. By Lemma 6.5 (2), we have three subcases to consider.

Subcase (i). $B$ is a parallelogram with vertices $\pm(3(2 m+1) /|k|, 6 /|k|)$ and $\pm(3(2 m+1+k) / 2|k|, 3 /|k|)$, for some integer $m$ and odd integer $k$, with $|k| \geq 5$.

Then, $3 B$ is a parallelogram with vertices $\pm(9(2 m+1) /|k|, 18 /|k|)$ and $\pm(9(2 m+1+k) / 2|k|, 9 /|k|)$. Note that the automorphism of $L$ which fixes $(2 m+1,2)$ and switches $(m, 1)$ and $(m+1,1)$ maps the fundamental polygon associated to the parameter $k$ to that associated to the parameter $-k$. Thus we can, and will, assume that $k \leq-5$.

If $k=-5$, then $3 B \cap L$ contains primitive elements $\pm(3 m+1,3)$, $\pm(3 m+2,3), \pm(2 m-3,2), \pm(2 m-1,2), \pm(2 m+1,2), \pm(m-3,1), \pm(m-2,1)$, $\pm(m-1,1), \pm(m, 1), \pm(m+1,1), \pm(m+2,1), \pm(1,0)$. Note that $(2 m+1,2)$ and $(m-2,1)$ are two strict boundary classes in $L$. As in Case I.1, one can show, using Theorem 2.3, that none of $\pm(3 m+1,3), \pm(3 m+2,3), \pm(2 m-3,2), \pm(m-3,1)$ can be a finite/cyclic surgery slope. Hence we have $\# \leq 6, \Delta \leq 5, n_{0} \leq 2$.

In a like manner, one can show that when $k \leq-7$, Main Claim I holds.

Subcase (ii). $\quad B$ is a parallelogram with vertices $\pm(3 m /|k|, 3 /|k|)$ and $\pm(3(m+k) / 2|k|, 3 / 2|k|)$, for some integers $m$ and $k$, with $|k| \geq 3$.

We may assume that $k \geq 3$. Then $3 B$ is a parallelogram with vertices $\pm(9 \mathrm{~m} / k$, $9 / k)$ and $\pm(9(m+k) / 2 k, 9 / 2 k)$. If $k=3$, then $3 B \cap L$ contains primitive elements $\pm(1,0), \pm(2 m-1,2), \pm(2 m+1,2), \pm(2 m+3,2), \pm(m-2,1), \pm(m-1,1), \pm(m, 1)$, $\pm(m+1,1), \pm(m+2,1), \pm(m+3,1), \pm(m+4,1)$; but note that $(m, 1)$ and $(m+3,1)$ are strict boundary classes in $L$. As in Case I.1, one can show, using Theorem 2.3, that none of $\pm(2 m-1,2), \pm(2 m+1,2), \pm(2 m+3,2), \pm(m-2,1), \pm(m+4,1)$ are finite/cyclic surgery slopes. Hence we obtain $\# \leq 4, \Delta \leq 3$ and $n_{0} \leq 1$. Similarly, one can show that Main Claim I holds for $k>3$.

Subcase (iii). $B$ has three pairs of vertices $\pm(3 m /(2 j+q), 3 /(2 j+q))$, $\pm(3(m+j) /(j+q), 3 /(j+q))$ and $\pm(3(m+j+q) /(j+2 q), 3 /(j+2 q))$, for some integers $m, j>0, q>0$, with $j+q \geq 3$.

The proof is analogous to that of subcases (i) and (ii), and we have $\# \leq 4, \Delta \leq 5$ and $n_{0} \leq 2$.

Case I.3. $s \geq 8$. Let $\alpha$ be a finite/cyclic surgery class. According to Theorem 2.3, when $s=8$ we have $\|\alpha\| \leq \frac{5}{2} s$, i.e. $\alpha \in \frac{5}{2} B$, and when $s>8$ we have $\|\alpha\| \leq \frac{11}{5} s$, i.e. $\alpha \in \frac{11}{5} B$.

Suppose now that $\alpha=(j, k), k \geq 0$, is chosen so that $k$ is maximal. Main Claim I is easily seen to hold when $k=0$, and so we take $k \geq 1$. Using the estimates from the previous paragraph, it follows from Lemma 6.4 that $k \leq 4$, and that if $k=3$ or $k=4$, then $B$ must contain points $(a, b)$ satisfying $|b|>1$. Assume that this is the case, and let $(m, 1)$ and $(m+1,1)$ be the integral classes of $L$ such that there 
are points $(a, 1) \in B$ with $m<a<m+1$. Note that $B \cap\{y \geq 1\}$ is bounded by the lines $y=1,(m+1) y=x+1$, and $m y=x-1$. It follows that $\frac{5}{2} B \cap\left\{y \geq \frac{5}{2}\right\}$ contains no primitive class with denominator 4 , and furthermore, that $(3 m+1,3)$ and $(3 m+2,3)$ are the only primitive classes there with denominator 3 . Thus $k \neq 4$, and if $k=3$, then $j=3 m+1$ or $3 m+2$. We show now that $k=3$ is impossible.

The points $\left(\frac{3 m+1}{2}, \frac{3}{2}\right)$ and $\left(\frac{3 m+2}{2}, \frac{3}{2}\right)$ lie on the previously determined bounding lines for $B \cap\{y \geq 1\}$, and thus each has norm at least $s$. Thus $\|(3 m+1,3)\|$, $\|(3 m+2,3)\| \geq 2 s$, and so as $s \geq 8$, if one of these classes is a finite/cyclic class, then Theorem 2.3 implies that (i) it is either of $D_{-}, Q_{-}, O$ - or $I$-type, and (ii) there is an index $q=2,3$ or 5 sublattice $\tilde{L}$ of $L$ such that it realizes the minimal norm amongst the non-zero elements of $\tilde{L}$. Consider first of all the case where $q=2$, and $(j, k)=(3 m+1,3)$. Then $2 s \leq\|(3 m+1,3)\| \leq\|(m+1,1)\|$, and thus $\left\|\left(\frac{m+1}{2}, \frac{1}{2}\right)\right\| \geq s$. Considering the line through $\theta$ and $\left(\frac{m+1}{2}, \frac{1}{2}\right)$, we see that $B \cap\{y>1\}=\emptyset$, a contradiction. A similar contradiction is obtained if we assume $q=2$ and $(j, k)=(3 m+2,3)$.

If we suppose now that $q=3$ and $(j, k)=(3 m+1,3)$, then we arrive at the impossible relation $2 s \leq\|(3 m+1,3)\| \leq\|(1,0)\|=s$. On the other hand, if $q=3$ and $(j, k)=(3 m+2,3)$, then $(2,0) \in \tilde{L}$, as is $(3,0)$. Hence $\theta \in \tilde{L}$, and therefore $s \geq 2 s$, a contradiction.

The only possibility left is $q=5$. In this event, $\alpha$ is an $I$-type class (by Theorem 2.3), and so $s+8 \geq\|\alpha\| \geq 2 s$, implying that $s=8$ and $\|\alpha\|=2 s$. If $(j, k)=(3 m+1,3)$, then $\left(\frac{3 m+1}{2}, \frac{3}{2}\right) \in \partial B$, and therefore the line segment, $B_{1}$ say, between this latter point and $-\theta$ is contained in $\partial B$. If $\Lambda$ is the vertex of $B$ whose $y$-coordinate is at least $\frac{3}{2}$, and which lies on the line containing $B_{1}$, then by Lemma $6.5(3)$, the only possibilities are $\Lambda=\left(\frac{3 m+1}{2}, \frac{3}{2}\right)$ or $\Lambda=(2 m+1,2)$. The former is impossible, as we have assumed that $\alpha$ is not a strict boundary slope, and the latter is also impossible by Lemma 6.4. The final case where $(j, k)=(3 m+2,3)$ is handled in an entirely analogous manner, and we conclude that $k$ cannot be 3 .

Consider now the case where $k=2$, say $\alpha=(2 m+1,2)$ for some integer $m$, and assume first of all that $\|\alpha\|>2 s$. Then, $\alpha$ is necessarily of type $O$, and minimizes the norm amongst the non-trivial elements in some sublattice of $L$ of index $q=2$ or 3 . Now $\theta$ and $\alpha$ are congruent $(\bmod 2)$, and therefore $q \neq 2$. Hence $q=3$, and so $2 s<\|(2 m+1,2)\| \leq\|(-m+1-3 p,-1)\|=\|(m-1+3 p, 1)\|$ for any integer $p$. Now, the line segments from $-\theta$ and $\theta$ to $\frac{2}{5} \alpha$ lie in $B$, and so it follows that $\|(m, 1)\|<2 s,\|(m+1,1)\|<2 s$. Then, the convexity of $B$ implies that $\|(m+i, 1)\|<\|(m+i+1,1)\|$ for each $i \geq 1$, and that $\|(m+i, 1)\|<\|(m+i-1,1)\|$ for each $i \leq 0$. Theorem $2.3(5)$ now shows that $(m+i, 1)$ is not a finite/cyclic surgery class, whenever $i \geq 3$ or $i \leq-2$. Finally, the fact that $2 s<\|(m-1,1)\|$ and $2 s<\|(m+2,1)\|$ implies that for $i \neq 1,\|(2 m+i, 2)\|>\frac{5}{2} s$, and so $(2 m+1,2)$ is the only possible finite/cyclic class with denominator greater than or equal to 2 . Therefore, we have $\# \leq 6$ and $\Delta \leq 3$ and $n_{0} \leq 2$.

We may therefore assume that for each finite/cyclic class of the form $\alpha=$ $(2 m+1,2)$, we have $\|\alpha\| \leq 2 s$. It follows that for each such class, $\left(m+\frac{1}{2}, 1\right) \in B$. Hence, if $(2 m+1,2)$ and $(2 n+1,2)$ are two such classes, then as $B$ contains no horizontal segment of length longer than $2,|n-m| \leq 2$. If $|n-m|=2$, then $B$ is necessarily the parallelogram with vertex pairs $\pm\left(m+\frac{1}{2}, 1\right)$ and $\pm\left(n+\frac{1}{2}, 1\right)$, contradicting our assumption that $(2 m+1,2)$ and $(2 n+1,2)$ are not strict boundary classes. 
Suppose now that $|n-m|=1$. Without loss of generality, we may take $(2 n+1,2)=(2 m-1,2)$. Then, the line segment from $\left(m-\frac{1}{2}, 1\right)$ to $\left(m+\frac{1}{2}, 1\right)$ lies in $B$, and therefore in $\partial B$. It follows that $\|(2 m-1,2)\|=\|(2 m+1,2)\|=2 s$ and, as $s \geq 8$, Theorem 2.3 implies that these two classes minimize the norm amongst the non-trivial elements in some sublattice (depending on the class) of $L$, of in$\operatorname{dex} q=2,3$ or 5 . Now $\theta$ is congruent to both $(2 m-1,2)$ and $(2 m+1,2)(\bmod$ 2 ), and so $q$ cannot be 2 . If $q=3$ for one of the classes, say $(2 m+1,2)$, then $\|(m-1,1)\| \geq 2 s$, and so $\left(\frac{m-1}{2}, \frac{1}{2}\right)$ is not an interior point of $B$. But this contradicts the fact that $\left(m-\frac{1}{2}, 1\right) \in B$, and so $q \neq 3$. It follows that $q=5$, and therefore, that both $(2 m \pm 1,2)$ are of $I$-type and further, that $s=8$. Now, neither $\left(\frac{m-1}{2}, \frac{1}{2}\right)$ nor $\left(\frac{m+1}{2}, \frac{1}{2}\right)$ are vertices of $B$, and so it follows from Lemma 6.5 (3), that $B$ is the parallelogram with vertex pairs $\pm(m-1,1)$ and $\pm(m+1,1)$. In this case, we obtain $\# \leq 6, \Delta \leq 5$ and $n_{0} \leq 2$.

Suppose now that there is exactly one finite/cyclic class $(2 m+1,2)$. We wish to determine which integral lattice points can possibly be finite/cyclic classes (which are not strict boundary classes). The triangle with vertices $-\theta, \theta$ and $\left(m+\frac{1}{2}, 1\right)$ lies entirely in $B$, and therefore (i) $\|(m, 1)\|,\|(m+1,1)\| \leq \frac{3}{2} s$, and (ii) the line segment from $(m-1,1)$ to $(m+2,1)$ lies in $\frac{5}{2} B$. Now, any horizontal line intersects $B$ in a segment of length no more than 2 , and thus from (ii) we see that $\frac{5}{2} B \cap$ $\{y=1\}$ is contained in the line segment between $(m-3,1)$ and $(m+4,1)$. Thus only $(m-3,1),(m-2,1),(m-1,1),(m, 1),(m+1,1),(m+2,1),(m+3,1)$, and $(m+4,1)$ can be finite/cyclic classes. Next, note that (ii) shows that $\left(\frac{2(m+1)}{5}, \frac{2}{5}\right) \in$ $B$, and so again, using the fact that any horizontal line intersects $B$ in a segment of length no more than 2 , we have that $\|(m-3,1)\| \geq \frac{5}{2} s$. Hence, if $(m-3,1)$ is a finite/cyclic class, then from Theorem 2.3, it would have to be $O$-type with an associated sublattice of index 2 or 3 . But either possibility would contradict the fact that by $(\mathrm{i})$, both $(m, 1)$ and $(m+1,1)$ have norms less than $2 s$. Thus $(m-3,1)$ is not a finite/cyclic class. In a similar way, $(m+4,1)$ is ruled out.

Suppose now that $(m-2,1)$ is a finite/cyclic class. Arguing as in the case we have just considered, we have $\|(m-2,1)\| \leq 2 s$. Then $\left(\frac{m-2}{2}, \frac{1}{2}\right) \in B$, and hence (i) $\|(m+3,1)\|>\frac{5}{2} s$, i.e. $(m+3,1)$ cannot be a finite/cyclic class, (ii) $\|(m-1,1)\|<2 s$, and (iii) $\|(m+2,1)\| \geq 2 s$. Now if $(m+2)$ is a finite/cyclic class, then using Theorem 2.3 and (ii), we see that the associated lattice must have index 5, and so it is of $I$-type. As $s \geq 8$, Theorem 2.3 (4) now forces $s=8$ and $\|(m-2,1)\|,\|(m+2,1)\|=2 s$. It follows that the line segments from $-\theta$ to $\left(\frac{m-2}{2}, \frac{1}{2}\right)$, and from $\theta$ to $\left(\frac{m+2}{2}, \frac{1}{2}\right)$, lie in $\partial B$ and do not contain a vertex. It is now a simple matter, using Lemma 6.5 (3), to list all the possible shapes for $B$, and to verify that Main Claim I holds in each case.

In an analogous fashion, it can be shown that Main Claim I holds when $(m+3,1)$ is a finite/cyclic class. Thus, we may assume that neither $(m-2,1)$ nor $(m+$ $3,1)$ are such classes. Then the list of finite/cyclic classes is contained amongst $\theta$, $(m-1,1),(m, 1),(m+1,1),(m+2,1),(2 m+1,2)$, and so Main Claim I holds.

The final case to be considered is when $k=1$, i.e. when all finite/cyclic classes, other than $\theta$, are integral. Now, all such classes lie in $\frac{5}{2} B \cap\{y=1\}$ when $s=8$, and in $\frac{11}{5} B \cap\{y=1\}$ when $s>8$. In particular, there are no more than six such integral classes, and if there are six, then $s=8$ and $\frac{5}{2} B \cap\{y=1\}$ is the line segment between $(m-2,1)$ and $(m+3,1)$ for some integer $m$. Main Claim I then holds in all cases, except perhaps when $s=8$ and $\frac{5}{2} B \cap\{y=1\}$ is the line segment between 
$(m-2,1)$ and $(m+3,1)$. Note then that (i) $B$ contains the parallelogram $P$ with vertex pairs $\left(\frac{2(m-2)}{5}, \frac{2}{5}\right),\left(\frac{2(m+3)}{5}, \frac{2}{5}\right)$, and (ii) $\|(m-2,1)\|=\frac{5}{2} s$. Now $(m-2,1)$ must be an $O$-type class with the associated sublattice having index $q=2$ or 3 . In either event, at least one of $(m, 1)$ and $(m+1,1)$, say $(m, 1)$, must have norm at least $\frac{5}{2} s$, and thus equal to $\frac{5}{2} s$. Hence $\left(\frac{2 m}{5}, \frac{2}{5}\right) \in \partial B$, and it follows that $B=P$. It is now easy to verify that this, the final case of Main Claim I, holds. Thus we are done.

Proof of Main Claim II. Here we shall consider the four cases $s=2, s=4, s=6$ and $s \geq 8$.

Case II.1. $s=2$. Then by Theorem 2.3, for any finite/cyclic surgery class $\alpha$, $\|\alpha\| \leq 7 s$, i.e. $\alpha \in 7 B$. By Lemma 6.6 (1), $B$ is a parallelogram with vertices $\pm(1, \overline{0})$ and $\pm(m / k, 1 / k)$ for some $k \geq 1$.

Suppose $k=1$. Then, $7 B$ is a parallelogram with vertices $\pm(7 \mathrm{~m}, 7)$ and $\pm(7,0)$. The slopes in $7 B \cap L$ are $\pm(m+i, 1), i=0, \pm 1, \pm 2, \pm 3, \pm 4, \pm 5, \pm 6$, $\pm(2 m+i, 2), i= \pm 1, \pm 3, \pm 5, \pm(3 m+i, 3), i= \pm 1, \pm 2, \pm 4, \pm(4 m+i, 4), i= \pm 1, \pm 3$, $\pm(5 m+i, 5), i= \pm 1, \pm 2$, and $\pm(6 m+1,6)$. As in case I.1, one can use Theorem 2.3 to show that only $\pm(2 m+i, 2), i= \pm 1, \pm(m+i, 1), i= \pm 1, \pm 2$ can possibly be finite/cyclic surgery slopes. Hence, we obtain $\# \leq 6, \Delta \leq 5, n_{0} \leq 2$ and $m_{0} \leq 4$. For $k \geq 2$, a similar analysis shows that Main Claim II holds.

Case II.2. $s=4$. Then by Theorem 2.3, for any finite/cyclic surgery class $\alpha$, $\|\alpha\| \leq 4 s$, i.e. $\alpha \in 4 B$. According to Lemma 6.6 (2), we have three cases to consider.

Subcase (i). $B$ is a parallelogram with vertices $\pm(1,0)$ and $\pm\left(\frac{2 m}{k+2}, \frac{2}{k+2}\right)$ for some integers $m$ and $k \geq 0$. Notice that $\theta$ and $(m, 1)$ are strict boundary classes in $L$.

Now $B$ is contained in the parallelogram $P$ with vertices $\pm(1,0)$ and $\pm(m, 1)$. The slopes in $3 P$ which are not strict boundary slopes satisfy $\# \leq 6, \Delta \leq 5$, $n_{0} \leq 2$ and $m_{0} \leq 4$. We now show that $4 P-3 P$ does not contain finite surgery class. Suppose otherwise that $\alpha \in 4 P-3 P$ is a finite/cyclic surgery class. Then by Theorem 2.3, $\alpha$ is an $O$-type class and $\|\alpha\| \leq\|\delta\|$, where $\delta$ lies in an index $q=2$ or 3 sublattice of $L$ which contains $\alpha$. We discuss the case when $q=3$, the case when $q=$ 2 being similar. Note that $\alpha \in\{ \pm(m-3,1), \pm(m+3,1), \pm(3 m-1,3), \pm(3 m+1,3)\}$. If $\alpha=(m+3,1)$, then $\|(m, 1)\| \geq\|(m+3,3)\|=4 s$. Thus $B$ is contained in a parallelogram with vertices $\left(\frac{m}{4}, \frac{1}{4}\right)$ and $\pm(1,0)$, and so, $4 B$ cannot contain $\alpha$, a contradiction. Similarly $\alpha \neq(m-3,1), \alpha \neq(3 m-1,3)$ and $\alpha \neq(3 m+1,3)$.

Subcase (ii). $B$ is a parallelogram with vertices $\pm(1,0)$ and $\pm\left(\frac{2(2 j+1)}{k+2}, \frac{4}{k+2}\right)$, for some integers $j$ and $k \geq 2$.

The proof is similar to that of $(i)$ and Main Claim II holds.

Subcase (iii). $B$ is a polygon with vertices $\pm(1,0), \pm\left(\frac{2 m}{k+2}, \frac{2}{k+2}\right), \pm\left(\frac{2(m+j)}{k+2}, \frac{2}{k+2}\right)$, for some integers $m, j, k$ with $j \geq 1$ and $k \geq j-1$.

If $k=0$, then $j=1$, and $4 B$ contains slopes $\pm(m+i, 1), i=-3,-2,-1,0,1,2,3$, $4, \pm(2 m+i, 2), i=-1,1,3, \pm(3 m+i, 3), i=-1,1,2,4, \pm(4 m+i, 4), i=1,3$. Using Theorem 2.3 , one can easily show that only the slopes, $\pm(m-1,1), \pm(m+2,1)$ and $\pm(2 m+1,2)$ can possibly be finite/cyclic surgery slopes. It follows that we have the estimates $\# \leq 3, \Delta \leq 3, n_{0} \leq 2$ and $m_{0} \leq 2$.

Similarly, one can prove that Main Claim II holds for the case $k \geq 1$.

Case II.3. $s=6$. Then by Theorem 2.3, for any finite/cyclic surgery classes $\alpha$, $\|\alpha\| \leq s+12=3 s$, i.e. $\alpha \in 3 B$, and if $\alpha$ is not an $O$-type class with norm larger 
than $s+8$, then $\|\alpha\| \leq s+8=\frac{7}{3} s$, i.e. $\alpha \in \frac{7}{3} B$. According to Lemma 6.6 (3), we have several subcases to consider.

Subcase (i). $B$ is contained in a parallelogram with vertices $\pm(1,0)$ and $\pm(m, 1)$, with $(m, 1)$ being a strict boundary class associated to a vertex of $B$.

Then, $3 B$ is contained in the parallelogram with vertices $\pm\left(\frac{7}{3}, 0\right)$ and $\pm\left(\frac{7 m}{3}, \frac{7}{3}\right)$. In this parallelogram the slopes are : $\pm(1,0), \pm(m-2,1), \pm(m-1,1), \pm(m, 1)$, $\pm(m+1,1), \pm(m+2,1), \pm(2 m-1,2)$ and $\pm(2 m+1,2)$. Noting that $(1,0)$ and $(m, 1)$ are strict boundary classes in $L$, we have $\# \leq 6, \Delta \leq 5, n_{0} \leq 2$ and $m_{0} \leq 4$.

Subcase (ii). $B$ is contained in a parallelogram with vertices $\pm(1,0)$ and $\pm\left(\frac{6 m}{5}, \frac{6}{5}\right)$ with $(m, 2)$ being a strict boundary class associated to a vertex of $B$.

Then $3 B$ is contained in the parallelogram with vertices $\pm(3,0)$ and $\pm(18 \mathrm{~m} / 5$, 18/5). This parallelogram contains slopes: $\pm(1,0), \pm(m-1,1), \pm(m, 1), \pm(m+1,1)$, $\pm(m+2,1), \pm(2 m+1,2), \pm(3 m+1,3)$ and $\pm(3 m+2,3)$. Now $(1,0)$ and $(2 m+1,2)$ are strict boundary classes in $L$ and further, using Theorem 2.3, it can be seen that $\pm(3 m+1,3), \pm(3 m+2,3)$ are not finite/cyclic surgery slopes. Hence, we obtain $\# \leq 4, \Delta \leq 3, n_{0} \leq 1$ and $m_{0} \leq 4$.

The remaining subcases given by Lemma 6.6 (3) may be similarly analyzed and Main Claim II holds in these cases.

Case II.4. $s \geq 8$. The discussion is similar to that of Case I.3, but is in fact much simpler because of the assumption that $\partial B \cap L$ consists entirely of vertices of $B$. The proof of Main Claim II is now complete.

\section{Proof of Theorem 1.2}

Let $r_{1}, r_{2}$ be two finite/cyclic slopes on $K$, and denote by $K_{1}, K_{2}$ the cores of their respective surgery solid tori. We may assume that the first Betti number of $M$ is 1 since otherwise both $r_{1}$ and $r_{2}$ are infinite cyclic surgery slopes, and thus, Theorem 1.2 follows from the cyclic surgery theorem. Note that under this assumption, at least one of $r_{1}$ and $r_{2}$ is necessarily a finite slope.

Fix an essential torus $T$ in $M=W-\operatorname{int} N(K)$. Now as we have just noted, $K$ admits a finite surgery, and therefore $T$ separates $M$. In the decomposition $M=M_{1} \cup_{T} M_{2}$, suppose that $M_{1}$ does not contain $\partial M$. By Haken's finiteness theorem [Hl, Lemma 13.2], we may assume that the essential torus $T$ was chosen so that $M_{1}$ is simple, i.e. it does not contain any essential torus. Therefore $M_{1}$ is either a Seifert fibred space or a hyperbolic manifold. Note further that if $M_{1}$ is a Seifert fibred space, then it has a Seifert fibration over a 2-disc with exactly 2 exceptional fibres.

By Dehn's lemma, $T$ is compressible in $M\left(r_{i}\right)=M_{1} \cup_{T} M_{2}\left(r_{i}\right)$, and thus, is compressible in $M_{2}\left(r_{i}\right)$. Hence, either $M_{2}\left(r_{i}\right)=D^{2} \times S^{1}$ is a solid torus, or $M_{2}\left(r_{i}\right)=$ $D^{2} \times S^{1} \# W_{i}$ is a connected sum of a solid torus and a closed 3-manifold $W_{i} \neq S^{3}$ with finite/cyclic fundamental group. If, for instance, $M_{2}\left(r_{2}\right)=D^{2} \times S^{1} \# W_{2}$, then by [Sch], $K_{1}$ is a cabled knot in $M_{2}\left(r_{1}\right)$, and $r_{2}$ is the slope of the cabling annulus. Then using [Go, Lemma 7.2 and Lemma 3.3], one can easily show that $M_{2}\left(r_{1}\right)$ is a solid torus. Therefore $M_{2}\left(r_{1}\right)$ and $M_{2}\left(r_{2}\right)$ cannot both be reducible, and so we may assume that $M_{2}\left(r_{1}\right)=D^{2} \times S^{1}$ is a solid torus.

Let $s_{i}$ be the slope on $\partial M_{1}$ which (considered as a curve) bounds a disc in $M_{2}\left(r_{i}\right)$. Select a slope $\lambda_{1}$ on $\partial M_{1}$ such that $s_{1}$ and $\lambda_{1}$ form a basis (after choosing orientations) of $H_{1}\left(\partial M_{1} ; \mathbf{Z}\right)$. Now recalling that $M_{2}\left(r_{1}\right)$ is a solid torus, there is a unique slope $\tau_{1}$ on $\partial M$ such that $\Delta\left(\tau_{1}, r_{1}\right)=1$, and that $\left[\tau_{1}\right]=w\left[\lambda_{1}\right]$ in $H_{1}\left(M_{2}\left(r_{1}\right) ; \mathbf{Z}\right)$, 
where $w$ is the winding number of $K_{1}$ in $M_{2}\left(r_{1}\right)$. Now $r_{1}$ and $\tau_{1}$ form a basis of $H_{1}(\partial M ; \mathbf{Z})$, and so, surgery slopes on $\partial M_{1}$ and on $\partial M$ will be parameterized by $\left(s_{1}, \lambda_{1}\right)$-coordinate system and $\left(r_{1}, \tau_{1}\right)$-coordinate system respectively.

If $\Delta\left(r_{1}, r_{2}\right)>1$, then by [CGLS, Theorem 2.0.1], $M_{2}$ is a cabled space. It follows from [Go, Lemma 7.2] that $K_{1}$ is cabled in $M_{2}\left(r_{1}\right)$ with winding number $w \geq 2$, that $M_{2}\left(r_{2}\right)$ is a solid torus, and that $w$ is coprime to the $r_{1}$-coordinate of $r_{2}$. Hence, using [Go, Lemma 3.3], we see that $\Delta\left(s_{1}, s_{2}\right)=\Delta\left(r_{1}, r_{2}\right) w^{2} \geq 8$. Applying Theorem 1.1 (1), we see that $M_{1}$ is Seifert fibred, and as we previously remarked, it admits a Seifert fibration over a 2-disc with exactly 2 exceptional fibres. Therefore, $M$ is a generalized 1-iterated torus knot exterior. This proves part (1) of the theorem.

We now go on to prove part (2). Assume that one of $r_{i}$ is an odd order cyclic surgery slope. By part (1), we may assume $\Delta\left(r_{1}, r_{2}\right)=1$.

Subcase (a). $M_{2}\left(r_{2}\right)$ is also a solid torus.

Then by [Ga1], $K_{i}$ is a 0 or 1-bridge braid knot in $M_{2}\left(r_{i}\right)$. In particular, the winding number $w$ of $K_{1}$ in $M_{2}\left(r_{1}\right)$ satisfies $w \geq 2$. By [Go, Lemma 7.2] and [Ga2, Lemma 3.2], the meridian coordinate of the slope $r_{2}$ is relatively prime to the winding number $w$ of $K_{1}$. Hence by [Go, Lemma 3.3], $\Delta\left(s_{1}, s_{2}\right)=w^{2} \geq 4$. Now applying Theorem $1.1(2)$, we see that $M_{1}$ is Seifert fibred.

We may assume that $M\left(r_{1}\right)$ has cyclic fundamental group of odd order. Now, $M_{1}$ admits a Seifert fibration over the 2-disc with exactly two exceptional fibres. For this fibration of $M_{1}$, a fibre on $\partial M_{1}$ represents a slope having distance one from the slope $s_{1}$. Hence we may now assume that the slope $\lambda_{1}$ on $\partial M_{1}$ was chosen to be a fibre.

If $K_{1}$ is a 0-bridge braid in $M_{2}\left(r_{1}\right)$, then $M$ is a generalized 1-iterated torus knot exterior. So we assume that $K_{1}$ is a 1-bridge braid in $M_{2}\left(r_{1}\right)$. By [Ga2, Lemma $3.2]$, only an integral slope of the form $\epsilon((t+j w) w+b+\delta)$ of $K$ can possibly yield a solid torus, where $t+j w$ is the twist number of $K_{1}$, with $1 \leq t \leq w-2(j$ being an integer), $b$ is the bridge width of $K_{1}$, with $1 \leq b \leq w-2, \epsilon= \pm 1$ and $\delta=0$ or 1. We claim that without loss of generality, we may take $w$ to be at least 5 . This is clearly the case when $b \geq 3$, so consider the cases where $b=1,2$. In the former case we apply [Ga2, Example 3.7] to see that $M_{2}$ is the union of two cabled spaces along a common toral boundary component, and hence, $M$ is a generalized 2 -iterated torus knot exterior. In the latter case, we apply [Ga2, Example 3.8 and Proposition 3.9] to see that under our hypotheses, $w$ is necessarily greater than, or equal to 5 .

By [Go, Lemma 3.3], $s_{2}=r_{2} / w^{2}$, i.e. $M\left(r_{2}\right)=M_{1}\left(r_{2} / w^{2}\right)$. Since $M_{1}\left(r_{2} / w^{2}\right)$ is Seifert fibred and has finite fundamental group (it cannot be infinite cylic), the indices of its exceptional fibres form a platonic triple. Now, the index of the fibre corresponding to the knot $K_{2}$ is

$$
\begin{aligned}
& \Delta\left(\lambda_{1}, r_{2} / w^{2}\right)=|(t+j w) w+b+\delta| \\
& \geq\left\{\begin{array}{lr}
w^{2}-t w-b-1 \geq w^{2}-(w-2) w-(w-2)-1=w+1>5 & \text { if } j \neq 0 \\
t \omega+b>5 & \text { if } j=0 .
\end{array}\right.
\end{aligned}
$$

Hence, the singular indices of the two exceptional fibres of the Seifert fibration of $M_{1}$ are necessarily 2 and 2, i.e. $M_{1}$ is the twisted $I$-bundle over the Klein bottle. But then, as $\pi_{1}\left(M_{1}\left(s_{1}\right)\right)=\pi_{1}\left(M\left(r_{1}\right)\right)$ is cyclic, it has even order, contradicting our hypotheses. This completes subcase (a). 
Remark. 1-bridge braid knots in a solid torus admitting at least two surgeries yielding solid tori have been classified [B].

Subcase (b). $M_{2}\left(r_{2}\right)=D^{2} \times S^{1} \# W_{2}$ is reducible.

By [Sch], $K_{1}$ is cabled, and $r_{2}$ is the slope of the cabling annulus. Let $K_{*} \subset$ $M_{2}\left(r_{1}\right)$ be the knot on which $K_{1}$ is cabled, and observe that $K_{*}$ admits two surgeries which yield a solid torus. Now, these two surgeries must have distance at least 2 by [Go, Lemma 3.3 and Lemma 7.2], and so applying [Ga1], [Ga2], $K_{*}$ must be a 0-bridge braid in $M_{2}\left(r_{1}\right)$. If the winding number $w_{*}$ of $K_{*}$ in $M_{2}\left(r_{1}\right)$ is at least 2, then $w \geq 4$, and so using [Go, Lemma 3.3], we see that $\Delta\left(s_{1}, s_{2}\right) \geq 4$. Thus, $M_{1}$ is Seifert fibred by Theorem 1.1 (2). $M$ is therefore a generalized 2-iterated torus knot exterior, and so we assume $w_{*}=1$, i.e. $K_{1}$ is a 0 -bridge braid in $M_{2}\left(r_{1}\right)$.

If the winding number $w$ of $K_{1}$ is greater than or equal to 3 , then again $\Delta\left(s_{1}, s_{2}\right) \geq$ 3 ([Go, Lemma 3.3]), and $M_{1}$ is therefore Seifert fibred by Theorem 1.1 (2). If $\omega=2$, then [Go, Lemma 7.2] shows that $W_{2}$ is the real projective 3 -space, and thus, $\pi_{1}\left(M\left(r_{2}\right)\right)$ is a cyclic group of order two. Thus, $M_{1}\left(s_{1}\right)=M\left(r_{1}\right)$ has cyclic fundamental group of odd order. But again, [Go, Lemma 3.3] implies $\Delta\left(s_{1}, s_{2}\right) \geq 2$, and so, the cyclic surgery theorem forces us to conclude that $M_{1}$ is Seifert fibred. Therefore, $M$ is a generalized 1-iterated torus knot exterior, which completes the proof of part (2).

Remark. From the above proof, we see that if there is no non-trivial non-integral finite surgery on any hyperbolic knot in $S^{3}$, then finite surgery on knots in manifolds of even order cyclic fundamental group, whose exteriors contain essential tori, can also be classified.

\section{Proof of Theorem 1.5}

In the proof of Theorem 1.5 we shall adopt the notation from $\S 5, \S 6$ and $\S 7$.

Proof of part (1) of Theorem 1.5. Suppose that $\alpha$ is a $D$-type or $Q$-type surgery class, and $\theta$ a cyclic surgery class satisfying $\Delta(\alpha, \theta) \geq 2$. Then by Lemma 6.7, neither of $\alpha$ and $\theta$ is a boundary class. Hence $\theta$ has minimal norm, and $\theta$ is not a vertex of the fundamental polygon $B$. As in $\S 6$, let $\theta$ and $\tau$ be a fixed basis of $L=H_{1}(\partial M)$. Let $\alpha=\theta^{m} \tau^{n}$. By Theorem $1.1(2),|n| \leq 2$. If $|n|=2$, then $m$ is odd, and by Theorem $2.3(2),\|\alpha\| \leq\|\theta\|=s$. Therefore, both $\alpha$ and $\theta$ are contained in the fundamental polygon $B$, and both are not vertices of $B$. Hence $\Delta(\alpha, \theta) \leq 1$, and a contradiction is obtained.

Suppose that $M=W-\operatorname{int} N(K)$ is a knot exterior in a connected closed orientable 3-manifold $W$, such that $H_{1}(M)=\mathbf{Z}[\xi] \oplus \mathbf{Z}_{p}[\zeta]$, with $p$ odd. Let $\pm \lambda \in L=H_{1}(\partial M)$ be the unique slope such that $H_{1}(M(\lambda) ; \mathbf{Q})=\mathbf{Q}$ (the rational longitude). Then for some integer $d, \lambda=d[\zeta]$ in $H_{1}(M)$. Let $\pm \mu \in L$ be a (fixed) slope such that $\mu$ and $\lambda$ form a basis of $L$. Slopes on $\partial M$ will be parameterized by this basis. Let $\mu=f[\xi]+g[\zeta]$ in $H_{1}(M)$. Then $f$ is necessarily an odd integer, since otherwise, the composition $H_{1}\left(\partial M ; \mathbf{Z}_{2}\right)=\mathbf{Z}_{2} \oplus \mathbf{Z}_{2} \rightarrow H_{1}\left(M ; \mathbf{Z}_{2}\right)=\mathbf{Z}_{2}$ could not be onto (the surjectivity of this homomorphism follows from the universal coefficient theorem and the Poincaré-Lefschetz duality theorem). Similarly, if $p$ is relatively prime to 3 , then $f$ is also.

Lemma 9.1. Let $\alpha=m[\mu]+n[\lambda] \in L$ be a primitive class. Then, the order of $H_{1}(M(\alpha))$ is mfp. Thus, 
(1) if $\alpha$ is an $O$-type surgery class, say $\pi_{1}(M(\alpha))=O_{48} \times \mathbf{Z}_{j}$, then $m=2 q$ and $|q f p|=j$;

(2) if $\alpha$ is a D-type surgery class, say $\pi_{1}(M(\alpha))=D\left(2^{k}, 2 l+1\right) \times \mathbf{Z}_{j}$ with $k \geq 2$, then $m=2^{k} q$ and $|q f p|=j$;

(3) if $\alpha$ is an even order $C$-type surgery class, then $m=2 q$ and $\pi_{1}(M(\alpha))=$ $\mathbf{Z}_{2|q f p|}$;

(4) if $p$ is relatively prime to 3 and $\alpha$ is a T-type surgery class, say $\pi_{1}(M(\alpha))=$ $T\left(8,3^{k}\right) \times \mathbf{Z}_{j}$, then $m=3^{k} q$ and $|q f p|=j$.

Proof. This is an elementary homological calculation which uses Lemma 5.1.

For a manifold $M$ given as above, let $p: \tilde{M} \rightarrow M$ be the two-fold cover associated to the map $\pi_{1}(M) \rightarrow H_{1}(M) \rightarrow \mathbf{Z}_{2}$. Note that $p^{-1}(\mu)$ is connected, and $p^{-1}(\lambda)$ consists of two components. We let $\tilde{\mu}=p^{-1}(\mu)$ and let $\tilde{\lambda}$ be a component of $p^{-1}(\lambda)$. Then $\tilde{\mu}$ and $\tilde{\lambda}$ form a basis of $H_{1}(\partial \tilde{M})$, and slopes on $\tilde{M}$ will be parameterized by this basis.

If $\alpha=m[\mu]+n[\lambda]$ is an $O$-type filling slope, say $\pi_{1}(M(\alpha))=O_{48} \times \mathbf{Z}_{j}$, then $m=2 q$ and $|q f p|=j$ (Lemma 9.1 (1)). The two-fold cover $\tilde{M} \rightarrow M$ extends to a free two-fold cover $\tilde{M}(q / n) \rightarrow M(2 q / n)$, with fundamental group $\pi_{1}(\tilde{M}(q / n))=T_{24} \times \mathbf{Z}_{j}$ (Lemma 5.2(4)). Then $H_{1}(\tilde{M}(q / n))=\mathbf{Z}_{3 j}$, and so it follows from Lemma 5.1, and the fact that $j$ is relatively prime to 3 , that $H_{1}(\tilde{M})=\mathbf{Z} \oplus \mathbf{Z}_{3 k}$ for some integer $k$ dividing $j$.

Suppose next that $K$ also admits an even order cyclic surgery, with slope $\beta=$ $2 a[\mu]+b[\lambda]$ and $\pi_{1}(M(\beta))=\mathbf{Z}_{2|a f p|}$ (Lemma 9.1 (3)). Then $\tilde{M}(a / b)$ is a free double cover of $M(2 a / b)$, which implies $\pi_{1}(\tilde{M}(a / b))=H_{1}(\tilde{M}(a / b))=\mathbf{Z}_{|a f p|}$. But on the other hand, from $H_{1}(\tilde{M})=\mathbf{Z} \oplus \mathbf{Z}_{3 k}$, we have $H_{1}(\tilde{M}(a / b))=\mathbf{Z}_{3|a k h|}$, for some integer $h$. Therefore 3 divides $f p$, and thus divides $j$, a contradiction. Hence we derive:

Lemma 9.2. Suppose that $M=W-$ int $N(K)$ is a knot exterior in a connected closed orientable 3-manifold $W$, such that $H_{1}(M)=\mathbf{Z} \oplus \mathbf{Z}_{p}$, with $p$ odd. If $M$ admits an $O$-type filling, then $M$ does not admit an even order cyclic filling.

Proof of part (2) of Theorem 1.5. (i) Suppose that $\alpha_{1}$ and $\alpha_{2}$ are two distinct $D$ type surgery classes. By Lemma $9.1, \Delta\left(\alpha_{1}, \alpha_{2}\right)$ is divisible by 4 , and so it is equal to 4 by Theorem 1.1 (1). By Lemma 6.7, neither $\alpha_{1}$ nor $\alpha_{2}$ is a boundary class.

Using $(\theta, \tau)$-coordinates of $\S 6$ and Theorem $2.3(1)$, we claim that both $\alpha_{1}$ and $\alpha_{2}$ are integral slopes in $L=\mathbf{Z}[\theta] \oplus \mathbf{Z}[\tau]$. For otherwise, we may assume that $\alpha_{1}=(m, n)$, with $n \geq 2$. Then by Lemma $6.4,\left\|\alpha_{1}\right\|>s$ and by Theorem 2.3 (2) (i), $\left\|\alpha_{i}\right\| \leq 2 s$. Thus, $n \leq 3$ by Lemma 6.4. If $n=2$, then Theorem 2.3 (2) (ii) implies that $\|(m, 2)\| \leq\|(1,0)\|=\|\theta\|=s$, a contradiction. If $n=3$, then as $\|(m, 3)\|<2 s,(m / 2,3 / 2)$ is contained in the fundamental polygon $B$, and thus, the line segments connecting $(m / 2,3 / 2)$ and $( \pm 1,0)$ are contained in $B$. But one of these two line segments contains an integral class $\theta_{1}$ of $L$. Thus $\theta_{1}$ is on the boundary of $B$, and is not a vertex of $B$. Note that $\Delta\left(\theta_{1}, \alpha_{1}\right)=2$. But this is impossible, by the same reason as that for showing $\Delta\left(\theta, \alpha_{1}\right) \neq 2$.

Hence, we may assume that $\alpha_{1}=(m, 1)$ and $\alpha_{2}=(m+4,1)$. Again by Theorem $2.3(2)(\mathrm{i}),\left\|\alpha_{1}\right\|=\left\|\alpha_{2}\right\| \leq 2 s$, and thus, $\left(\frac{m}{2}, \frac{1}{2}\right) \in B$ and $\left(\frac{m}{2}+2, \frac{1}{2}\right) \in B$. Therefore, the parallelogram $P$ with vertices $\left(\frac{m}{2}, \frac{1}{2}\right)$ and $\left(\frac{m}{2}+2, \frac{1}{2}\right)$ is contained in $B$. Now $\pm \theta= \pm(1,0) \in \partial P$, from which it follows that the line segment 
through $\left(\frac{m}{2}, \frac{1}{2}\right)$ and $(-1,0)$, as well as that through $\left(\frac{m}{2}+2, \frac{1}{2}\right)$ and $(1,0)$, lie in $\partial B$. In particular, $\|(m, 1)\|=\|(m+4,1)\|=2 s$. But we have already observed that neither $\left(\frac{m}{2}, \frac{1}{2}\right)$ nor $\left(\frac{m}{2}+2, \frac{1}{2}\right)$ can be vertices of $B$. Hence we must have $\|(m+2,1)\|<\|(m, 1)\|=\|(m+4,1)\|$. But this contradicts Theorem 2.3 (2) (ii).

(ii) Suppose that $\alpha$ is a $D$-type surgery class, and $\theta$ is an even order cyclic surgery class. Then by Lemma $9.1(2)-(3), \Delta(\alpha, \theta) \geq 2$, which contradicts part (1) of Theorem 1.5.

(iii) Follows from part (1) of Theorem 1.5 and subcase (i).

Proof of part (3) of Theorem 1.5. (i) This is a special case of Lemma 9.2.

(ii) We use notation set up in proving Lemma 9.2. Suppose that $\alpha=m[\mu]+n[\lambda]$ is an $O$-type filling class, and $\beta=m_{1}[\mu]+n_{1}[\lambda]$ is a cyclic filling class. By Lemma 9.1 (1), $m$ is an even integer and by Lemma 9.1 and Lemma $9.2, m_{1}$ is an odd integer. Therefore $\Delta(\alpha, \beta)$ is an odd integer. Hence by Theorem $1.1(2), \Delta(\alpha, \beta)=1$.

(iii) By Lemma 9.1 (1), any two distinct $O$-type slopes have distance divisible by 4, and hence by Theorem 1.1 (1), distance equal to 4 . Suppose that $M$ admits three $O$-type slopes $\alpha_{1}, \alpha_{2}, \alpha_{3}$. By Lemma 9.1 (1), we may write their $\mu, \lambda$-coordinates as $\left(2 q_{1}, n_{1}\right),\left(2 q_{2}, n_{2}\right),\left(2 q_{3}, n_{3}\right)$, where $q_{1}, q_{2}, q_{3}$ are all odd integers. Then we have the following three identities: (i) $2 q_{1} n_{2}-2 q_{2} n_{1}= \pm 4$, (ii) $2 q_{2} n_{3}-2 q_{3} n_{2}= \pm 4$, and (iii) $2 q_{3} n_{1}-2 q_{1} n_{3}= \pm 4$. Multiplying identity (i) by $q_{3}$, identity (ii) by $q_{1}$, identity (iii) by $q_{2}$, and then summing, we arrive at an equation of the form $0=4 Q$, where $Q$ is odd. This is an impossibility, and therefore, there are no more than two $O$-type slopes on $M$, and if there are two, their distance is 4 .

(iv) If $\alpha$ is an $O$-type slope and $\beta$ a $D$-type slope, then by Lemma 9.1 (1-2), $\Delta(\alpha, \beta) \equiv 2(\bmod 4)$. Hence by Theorem $1.1(1), \Delta(\alpha, \beta)=2$.

Proof of part (4) of Theorem 1.5. By Lemma 9.1 (4), any two distinct T-type slopes have distance at least 3 . Hence by Theorem 1.1, all $T$-type slopes have mutual distance exactly 3 . An argument analogous to that given in the proof of part (3) (iii) of Theorem 1.5 shows that there are no more than two $T$-type slopes.

\section{EXAMPLES}

The $(-2,3,7)$-pretzel knot admits two non-trivial cyclic surgeries, namely the surgeries with slopes 18 and 19 (discovered by Fintushel and Stern). This knot also admits an $I$-type surgery, namely the surgery with slope 17 [BH]. Applying the results of this paper and those of [CGLS] and [HO], we can show

Example 10.1. The $(-2,3,7)$-pretzel knot admits exactly 4 finite surgeries.

Proof. The $(-2,3,7)$-pretzel knot has exactly four boundary slopes, namely the slopes $0,16,20$ and $37 / 2[\mathrm{HO}]$. We shall use the notation developed in $\S 6$ where we specify $\theta$ to be the meridian class of the knot. Then, the cyclic surgery classes $\pm(1,0), \pm(18,1)$ and $\pm(19,1)$ are contained in the boundary of the fundamental polygon $B$, and they are not vertices of $B$. With the above data, it is easy to see that there are points $(a, b) \in B$ with $|b|>1$. By Lemma 6.5, the minimal norm $s$ is at least 8 . Hence $\|\alpha\| \leq 2 s$ for any finite surgery class $\alpha$, by Theorem 2.3.

It follows that the only possible points in $2 B \cap L \cap\{y=1\}$ are $(16,1),(17,1)$, $(18,1),(19,1),(20,1)$ and $(21,1)$. The only primitive element in $2 B \cap L \cap\{y=2\}$ is $(37,2) ; 2 B \cap L \cap\{y \geq 3\}$ contains no finite surgery class by Theorem 1.1 (2). But $(37,2),(16,1)$ and $(20,1)$ are boundary classes, and thus, by Lemma 6.7 , none of them can be a finite surgery class (each of them has distance two with at least one 
of the four finite surgery classes of $K)$; as 3 divides $21,(21,1)$ cannot be an $I$-type surgery class, and as 2 does not divide 21 , it is neither a $D$-type nor an $O$-type surgery class. Thus it is not a finite surgery class by Theorem 2.3.

Using the Montesinos trick, Bleiler and Hodgson have produced some examples of finite non-cyclic surgery on hyperbolic knots. We exhibit here four hyperbolic knots in $S^{3}$, each of which admits a finite non-cyclic surgery, and a non-trivial cyclic surgery, through the use of the same method. Recall that the double branched cover of $S^{3}$, with branched set in $S^{3}$ a Montesinos link of type $\left(e, \alpha_{1} / \beta_{1}, \ldots, \alpha_{n} / \beta_{n}\right)$, is a Seifert fibred space over $S^{2}$, with Seifert invariants $\left\{e ; \alpha_{1} / \beta_{1}, \ldots, \alpha_{n} / \beta_{n}\right\}$ [BZ], and that every surgery on a strongly invertible link yields a manifold which is a double branched cover of $S^{3}$ branched over a link in $S^{3}$ [Mon]. The trick to obtain a finite non-cyclic surgery on a hyperbolic knot is to find a strongly invertible link $L \subset S^{3}$, such that some surgery on $L$ yields a manifold which is a double cover of $S^{3}$, branched over a Montesinos link of type $\left(e ; \alpha_{1} / \beta_{1}, \alpha_{2} / \beta_{2}, \alpha_{3} / \beta_{3}\right)$ with $\alpha_{1}, \alpha_{2}, \alpha_{3}$ forming a platonic triple (we may assume that $\alpha_{i}>0$ for each $i$ ), i.e. $\frac{1}{\alpha_{1}}+\frac{1}{\alpha_{2}}+\frac{1}{\alpha_{3}}>1$, and that on the other hand, the surgery on $L$ may be transferred, using Kirby-Rolfsen calculus, to a surgery on a hyperbolic knot $K \subset S^{3}$. We refer to [Mon] for details on how to construct the branched link in $S^{3}$ corresponding to a surgery on a strongly invertible link.

Example 10.2. Let $K_{n} \subset S^{3}$ be the knot with the surgery description shown in Figure 1 (a). Then, the manifold obtained by $9 n+1$ surgery on $K_{n}$ is a Seifert fibred space over $S^{2}$, with Seifert invariants $\{0 ; 5 / 2,-2,(n-1) / n\}$. In particular, $K_{3}$ is a hyperbolic knot which admits a $D$-type finite surgery with slope 28 (the fundamental group of the resulting manifold is $D_{20} \times \mathbf{Z}_{7}$ ); $K_{4}$ is a hyperbolic knot which admits an $I$-type surgery with slope 37 (the fundamental group of the resultant manifold is $\left.I_{120} \times \mathbf{Z}_{37}\right)$.

Proof. Figure 1 shows the construction of the branched link in $S^{3}$ corresponding to the surgery on the link given in Figure 1 (a).

Note that $K_{n}$ comes from [FS, §4], where it is shown that each $K_{n}$ admits a cyclic surgery with slope $9 n$. In [Ma], it is shown that each $K_{n},|n| \geq 2$, is neither a torus knot nor an iterated torus knot [Ma]. Therefore, Corollary 1.4 shows that each $K_{n},|n| \geq 2$, is a hyperbolic knot.

Note that $K_{ \pm 2}$ is the $(\mp 2, \pm 3, \pm 7)$-pretzel knot, and $K_{ \pm 1}$ is the $( \pm 5,2)$-torus knot.

Similarly, one can show that $9 n-1$ surgery on $K_{n}$ yields the Seifert fibred space over $S^{2}$, with Seifert invariants $\{0 ;-5 / 2,2,(-n-1) / n\}$.

Example 10.3. Let $K_{n}^{*} \subset S^{3}$ be the knot with the surgery description shown in Figure 2 (a). Then, the manifold obtained by $16 n-1$ surgery on $K_{n}^{*}$ is the Seifert fibred space over $S^{2}$, with Seifert invariants $\{0 ; 3 / 2,-5 / 3,(n-1) / n\}$. In particular, $K_{3}^{*}$ is a hyperbolic knot which admits an $I$-type surgery (the fundamental group of the resultant manifold is $\left.I_{120} \times \mathbf{Z}_{47}\right)$.

Proof. Figure 2 shows the construction of the branched link in $S^{3}$ corresponding to the surgery on the link given in Figure 2 (a). 
(a)

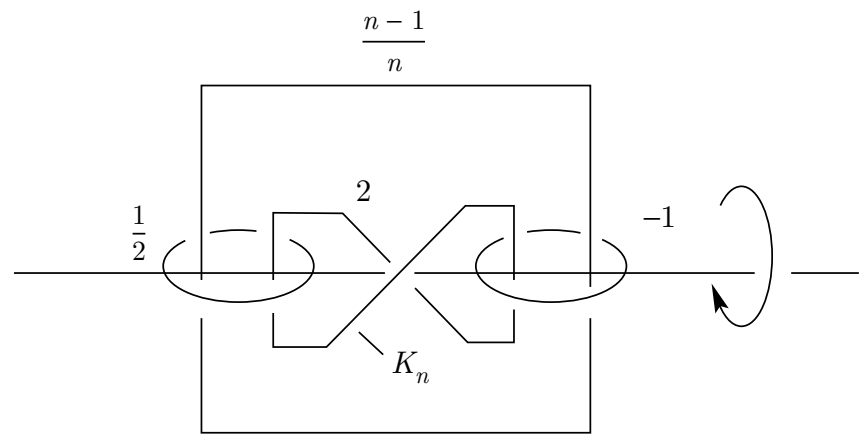

(b)

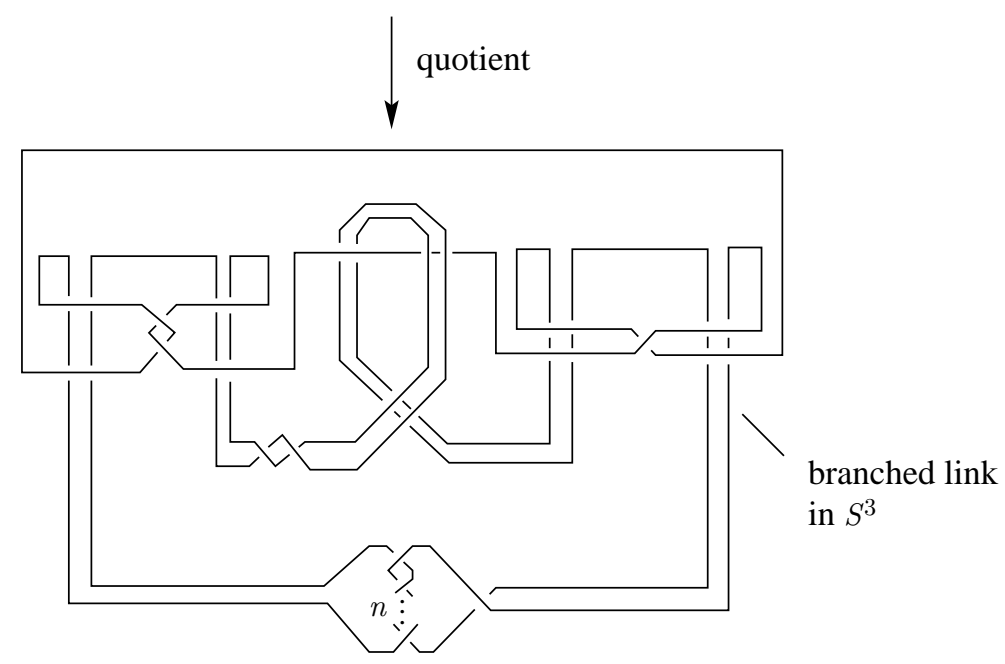

(c)

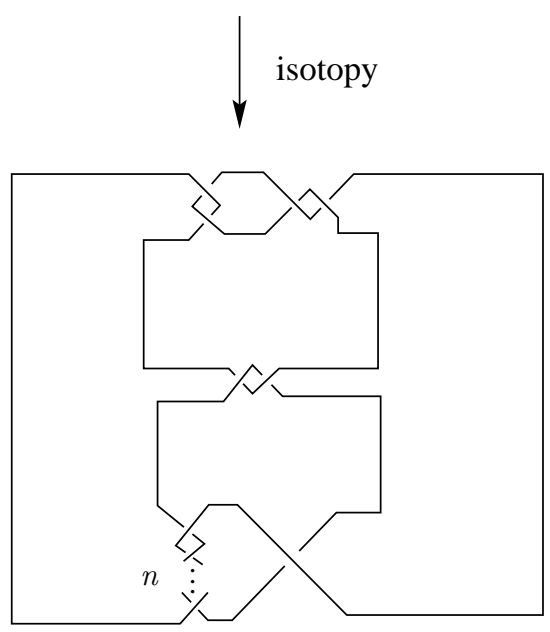

Figure 1 
(a)

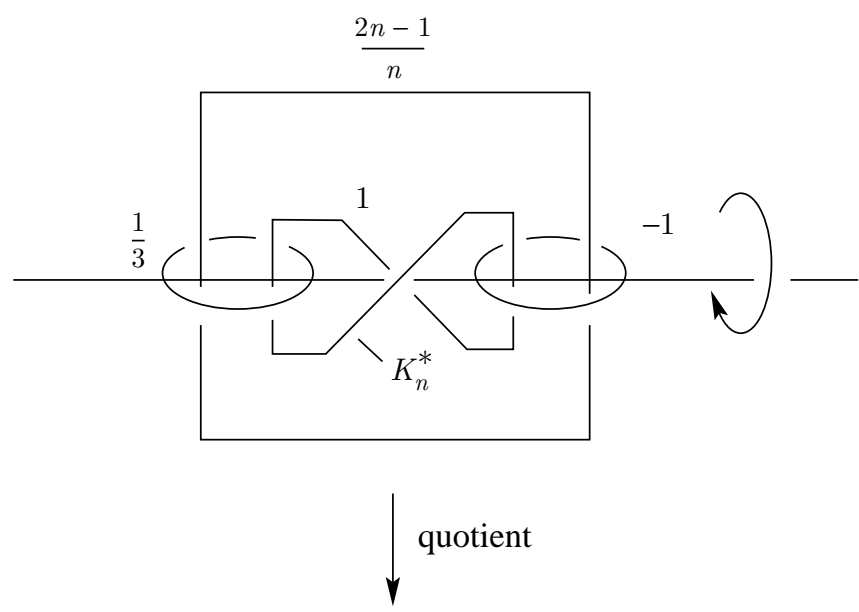

(b)

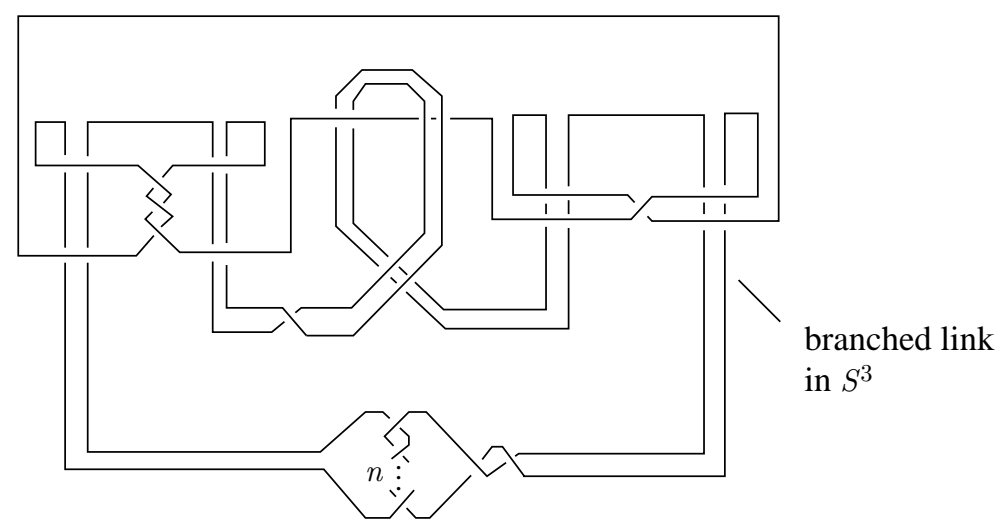

(c)

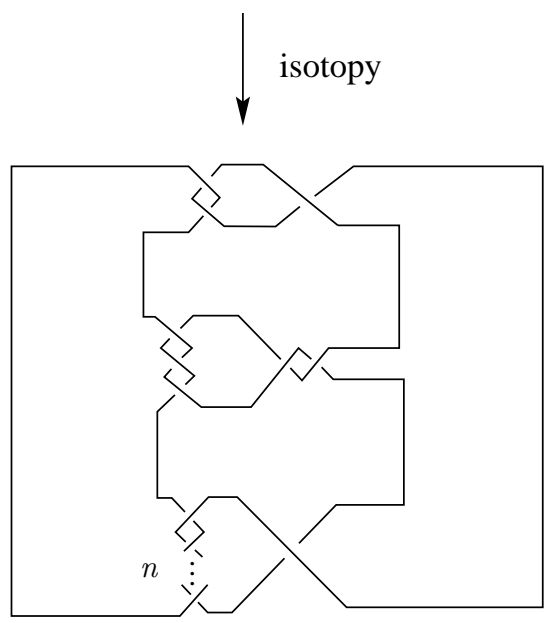

FiguRE 2 
Note that each $K_{n}^{*}$ is shown in [Ma] to admit a cyclic surgery with slope $16 n-2$. It is also shown there that each $K_{n}^{*}, n \neq 0,1$, is neither a torus knot nor an iterated torus knot. Therefore each $K_{n}^{*}, n \neq 0,1$, is a hyperbolic knot, by Corollary 1.4.

Note that $K_{1}^{*}$ is the $(3,5)$-torus knot, $K_{-1}^{*}$ is the $(2,-3,-7)$-pretzel knot, and $K_{2}^{*}$ admits two non-trivial cyclic surgeries.

Example 10.4. Let $K_{n}^{\#} \subset S^{3}$ be the knot with the surgery description shown in Figure 3 (a). Then the manifold obtained by $2(2 n+1)^{2}+1$ surgery on $K_{n}^{\#}$ is the Seifert fibred space over $S^{2}$, with Seifert invariants $\{0 ;-n /(n+1),-3 / 2$, $-(n+1) / n\}$. In particular, $K_{2}^{\#}$ is a hyperbolic knot which admits a $T$-type surgery with slope 51 (the fundamental group of the resultant manifold is $T_{24} \times \mathbf{Z}_{17}$ ).

Proof. Figure 3 shows the construction of the branched link in $S^{3}$ corresponding to the surgery on the link given in Figure 3 (a).

Note that each $K_{n}^{\#}$ is shown in [Ma] to admit a cyclic surgery with slope $2(2 n+1)^{2}$. It is also known there that each $K_{n}^{*}, n \neq 0,-1$, is neither a torus knot, nor an iterated torus knot. Therefore each $K_{n}^{\#}, n \neq 0,1$, is a hyperbolic knot by Corollary 1.4 .

Note that $K_{-2}^{\#}$ is the $(-2,3,7)$-pretzel knot, $K_{-1}^{\#}$ is the trivial knot, $K_{1}^{\#}$ is also the $(-2,3,7)$-pretzel knot, and $K_{-3}^{\#}$ and $K_{2}^{\#}$ are the same knot.

Example 10.5. This example is due to Weeks [W]. Let $L$ be the Whitehead link (Figure $4(\mathrm{a})$ ) with standard meridian-longitude coordinates. Let $Y=S^{3}-$ $\operatorname{int} N(L)$, and let $M$ be the manifold obtained by Dehn filling along one of the components of $\partial Y$, with slope 5 . Then, $M$ is a hyperbolic manifold with $H_{1}\left(M_{1}, \mathbf{Z}\right)=$ $\mathbf{Z} \oplus \mathbf{Z}_{5}$, and $M$ admits 5 finite Dehn fillings, with maximal mutual distance 3 . More explicitly, $M$ has three cyclic, and two T-type Dehn fillings: $M(1 / 0)=M(1)=$ $L(5,1), M(2)=L(10,3), M(3)=M(3 / 2)$ is the space form with fundamental group $T_{24} \times \mathbf{Z}_{5}$ (this can be verified using Montesinos trick). Besides, $M(0)$ is a torus bundle over the circle with Sol-geometry, $M(4)=M(4 / 3)$ is a union along a torus, of the twisted $I$-bundle over the Klein bottle and the exterior of the trefoil knot. All the remaining Dehn fillings on $M$ yield hyperbolic manifolds (cf. also $[\mathrm{MF}])$.

For this manifold $M$, we can determine explicitly the fundamental polygon $B$ of the norm $\|\cdot\|$ on $H_{1}(\partial M ; \mathbf{R})$, and the minimal norm $s$ of a non-zero element of $H_{1}(\partial M ; \mathbf{Z})$, defined in $\S 2$. Note that the manifold $M(-1)$ can also be obtained by 5 -surgery on the figure-eight knot (by Kirby calcuclus on the Whitehead link), and thus, $M(-1)$ is a non-Haken hyperbolic manifold [T3]. By Lemma 6.7, we see that none of the slopes $1,2,3,3 / 2$ and $1 / 0$ is a boundary slope (each of these slopes has distance at least 2 from one of the slopes -1 and $3 / 2$ ). Hence, all three of the cyclic slopes are contained in $\partial B$, and they are not vertices of $B$. We may therefore use the standard meridian-longitude coordinates as the $(\theta, \tau)$-coordinates of $\S 6$. We claim that the fundamental polygon $B$ is the one shown in Figure $4(\mathrm{~b})$, and that the minimal norm $s$ is 8 .

In fact, it follows from Lemma 6.5 that $s$ is at least 8 . Since $\partial B$ contains no more than three slopes which are not vertices, neither of the $T$-type classes $(3,1)$ and $(3,2)$ can lie in $B$. Thus, we have $s<\|(3,1)\|=\|(3,2)\| \leq s+4$ by Theorem 2.3 (3). Now the equality $\|(3,1)\|=\|(3,2)\|$ implies that $3 s / 2=\|(3,3 / 2)\| \leq\|(3,1)\|=\|(3,2)\|$. 
(a)

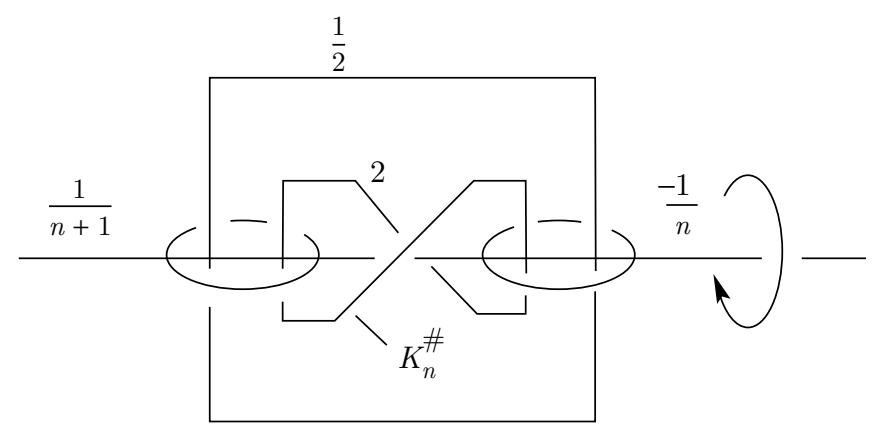

quotient

(b)

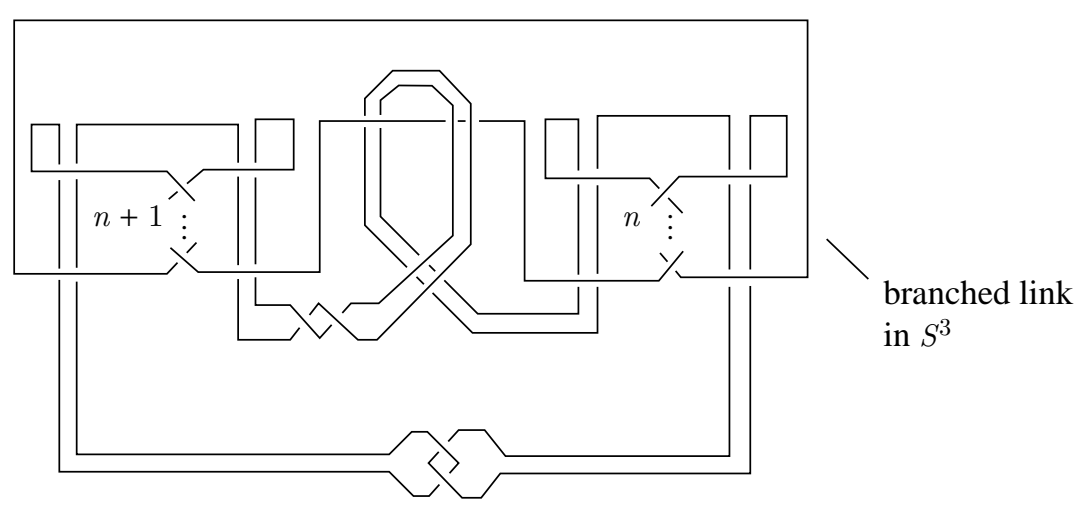

(c)

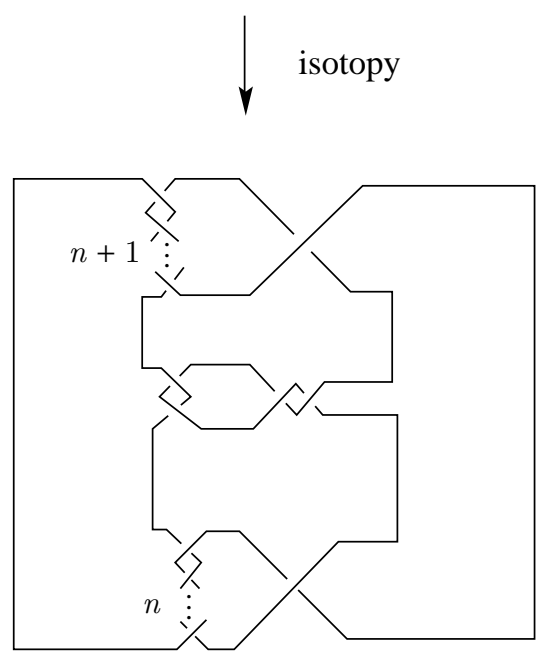

Figure 3 


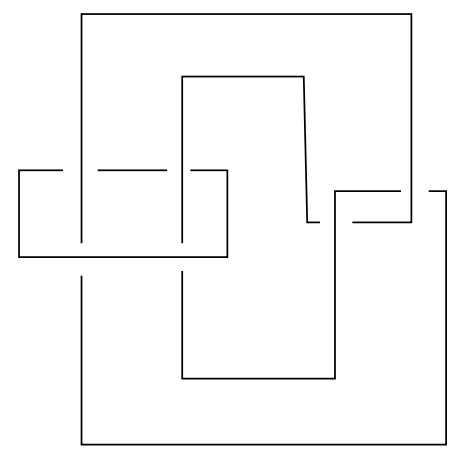

(a)

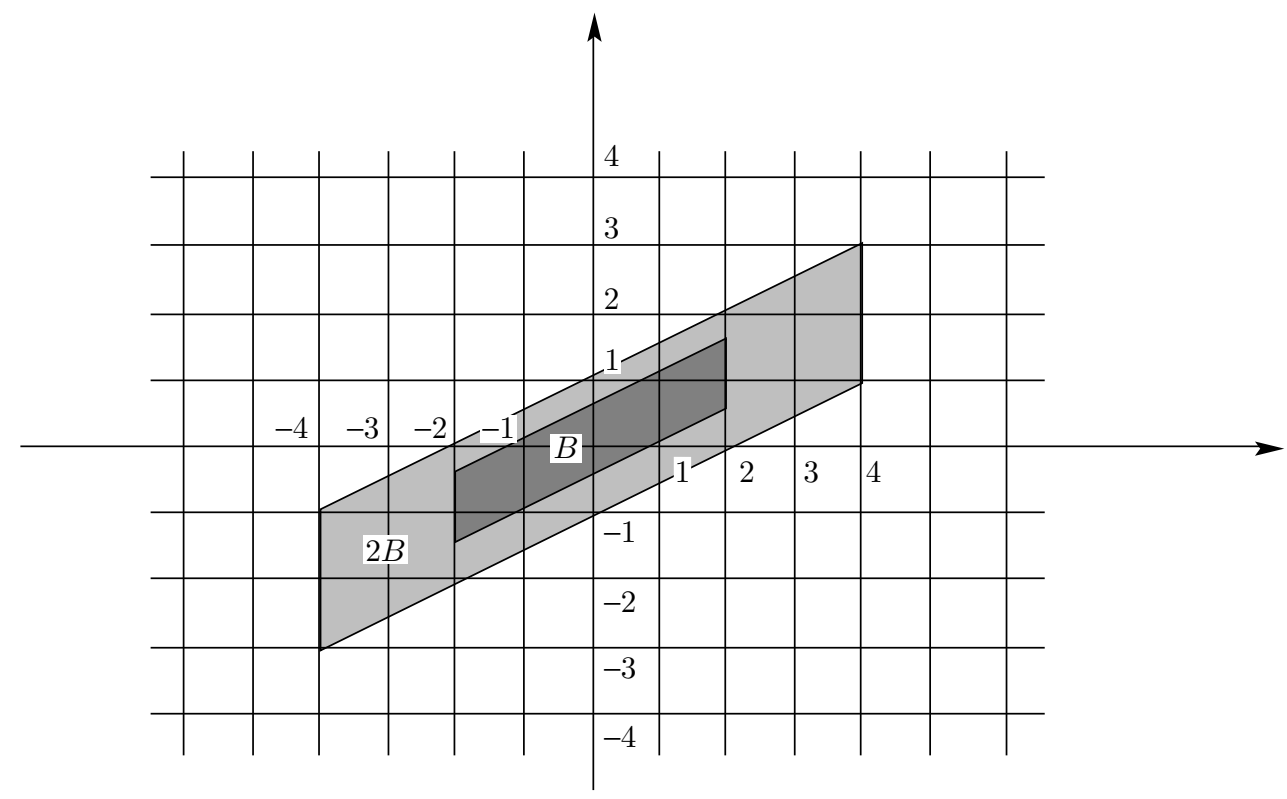

(b)

Figure 4

Hence $s+4 \geq 3 s / 2$, i.e. $s \leq 8$. Thus $s=8$ and $\|(3,1)\|=\|(3,2)\|=3 s / 2$. Now, combining the above data with Lemma 6.5 , we see that $B$ must be the parallelogram shown in Figure 4 (b).

It is interesting to note that all non-hyperbolic filling slopes lie on the region $2 B$, and that the norms of filling slopes can be ordered in the following pattern: the norms of cyclic filling slopes $<$ the norms of finite, non-cyclic filling slopes $<$ the norms of slopes resulting manifolds with essential torus $<$ the norms of hyperbolic filling slopes. 
The following example shows that Theorem 1.2 (1) is sharp.

Example 10.6. Let $M_{1}$ be the twisted $I$-bundle over the Klein bottle. Then each Dehn filling on $\partial M_{1}$, with only one exception, produces a manifold of finite/cyclic fundamental group, which is either an even order cyclic or a $D$-type group. The exceptional filling slope on $\partial M_{1}$ (which yields $R P^{3} \# R P^{3}$ ) has distance one with any cyclic filling slope on $\partial M_{1}$. Let $W=M_{1} \cup V$ be a manifold obtained by filling $\partial M_{1}$ with a solid torus $V$, such that $W$ has (even) cyclic fundamental group.

It is shown in [Ga1], [Ga2] and [B] that there is a (unique) 1-bridge braid knot $K$ in a solid torus $D^{2} \times S^{1}$, such that $K$ is not cabled, and $K$ admits three surgery slopes $r_{i}, i=1,2,3$, yielding solid tori. Note that the knot $K$ has winding number 7 in $D^{2} \times S^{1}$, and $M_{2}=D^{2} \times S^{1}-\operatorname{int} N(K)$ is a hyperbolic manifold.

Now, we embed the solid torus $D^{2} \times S^{1}$ into $W$ by a homomorphism of the two solid tori $D^{2} \times S^{1}$ and $V$. Then $K$, as a knot in $W$, admits three finite surgeries (the three surgeries $r_{i}$ on $K$ are equivalent to three fillings $s_{i}$ on $\partial M_{1}$, and none of $s_{i}$ can be the above exceptional filling slope on $\partial M_{1}$, by Gordon's transformation formula [Go]). Also, $M=W-\operatorname{int} N(K)=M_{1} \cup_{\partial M_{1}} M_{2}$ contains the essential torus $\partial M_{1}$, but is not a generalized $n$-iterated torus knot exterior (this follows from the well known torus decomposition theorem of Jaco-Shalen-Johannson, since $M_{2}$ is hyperbolic).

\section{REFERENCES}

[B] J. Berge, The knots in $D^{2} \times S^{1}$ which have non-trivial Dehn surgery that yield $D^{2} \times S^{1}$, Topology and its Application 38 (1991) 1-19. MR 92d:57005

$[\mathrm{BH}] \quad$ S. Bleiler and C. Hodgson, Spherical space forms and Dehn fillings, preprint.

$[\mathrm{BN}] \quad \mathrm{S}$. Boyer and A. Nicas, Varieties of group representations and Cassson's invariant for rational homology 3-spheres, Trans. Amer. Math. Soc. 322 (1990) 507-522. MR 92a:57020

[BZ] G. Burde and H. Zieschang, Knots, de Gruyter 1985. MR 87b:57004

[CGLS] M. Culler, C. M. Gordon, J. Luecke and P.B. Shalen, Dehn surgery on knots, Ann. of Math. 125 (1987) 237-300. MR 88a:57026

[CS] M. Culler and P.B. Shalen, Varieties of group representations and splitting of 3-manifolds, Ann. of Math. 117 (1983) 109-146. MR 84k:57005

[FS] R. Fintushel and R. Stern, Constructing lens spaces by surgery on knots, Math. Z. 175 (1980) 33-51. MR 82i:57009a

[Ga1] D. Gabai, Surgery on knots in solid tori, Topology 28 (1989) 1-6. MR 90h:57005

[Ga2] , 1-bridge braids in solid tori, Topology and its Appli. 37 (1990) 221-235. MR 92b:57011

[Ga3] , Foliations and the topology of 3-manifolds. III. J. Diff. Geo. 26 (1987) 479-536. MR 89a:57014b

[Gl] W. Goldman, The symplectic nature of fundamental groups of surfaces, Advances in Math. 54 (1984) 200-225. MR 86i:32042

[Go] C.M. Gordon, Dehn surgery and satellite knots, Trans. Amer. Math. Soc. 275 (1983) 687-708. MR 84d:57003

[Hl] J. Hempel, 3-manifolds, Ann. of Math Studies 86, (1976). MR 54:3702

[Hf] H. Hopf, Zum Clifford-Kleinschen raumproblem, Math. Ann. 95 (1925-26) 313-319.

[HO] A. Hatcher and U. Oertel, Boundary slopes for Montesinos knots, Topology, 28 (1989) 453-480. MR 91e:57016

[J] W. Jaco, Lectures on three-manifolds topology, CBMS Regional Conf. Ser. Math. 43 (1980). MR 81k:57009

[JM] D. Johnson and J. Millson, Deformation spaces associated to compact hyperbolic manifolds, Discrete Groups in Geometry and Analysis, Progress in Mathematics Vol. 67 (1987) 48-106. MR 88j:22010

[Ma] N. Maruyama, On Dehn surgery along a certain family of knots, J. of Tsuda College 19 (1987) 261-280. MR 88g:57011 
[MF] S. V. Matveev and A. T. Fomenko, Constant energy surfaces of Hamiltonian systems, enumeration of three-dimensional manifolds in increasing order of complexity, and computation of volumes of closed hyperbolic manifolds, Russian Math. Surveys 43 (1988) 3-24. MR 90a:58052

[Mi] J. Milnor, Groups which act on $S^{n}$ without fixed points, Amer. J. Math. 79 (1957) 623631. MR 19:761d

[Mo] L. Moser, Elementary surgery along a torus knot, Pacific J. Math. 38 (1971) 737-745. MR 52: 4287

[Mon] J. Montesinos, Surgery on links and double branched coverings of $S^{3}$, Annals of Math. Studies 84 (1975) 227-260. MR 52:1699

[Mu] D. Mumford, Algebraic geometry I: complex projective varieties, Grundlehren der Math. Wiss. 221, Springer-Verlag, 1976. MR 56:11992

[P] R. Palais, On the existence of slices for actions of non-compact Lie groups, Ann. Math. 73 (1961) 295-323. MR 23:A3802

[Sch] M, Scharlemann, Producing reducible 3-manifolds by surgery on a knot, Topology 29 (1990) 481-500. MR 91i:57003

[Se] J. Serre, Linear representations of finite groups, Springer-Verlag, 1977. MR 56:8675

[Sh] I. Shafarevich, Basic algebraic geometry, Die Grundlehren der mathematischen Wissenschaften, Band 213, Springer-Verlag, New York, 1974. MR 56:5538

[Ta] D. Tanguay, Chirurgie finie et noeuds rationnels, doctoral dissertation, UQAM, 1995.

[T1] W. Thurston, Three-dimensional manifolds, Kleinian groups, and hyperbolic geometry, Bull. Amer. Math. Soc. 6 (1982), 357-381. MR 83h:57019

[T2] Hyperbolic structure on 3-manifolds I: Deformation of acylindrical manifolds, Ann. Math. 124 (1986) 203-246. MR 88g:57014

[T3] The geometry and topology of 3-manifolds, Lecture notes, Princeton University, 1977.

[W] J. Weeks, Ph.D. thesis, Princeton University 1985.

[Wl] A. Weil, Remarks on the cohomology of groups, Ann. of Math. 80 (1964), 149-157. MR 30:199

[Wo] J. A. Wolf, Spaces of constant curvature, McGraw-Hill Series in Higher Mathematics, 1967. MR 36:829

[Z] X. Zhang, On property I for knots in $S^{3}$, Trans. Amer. Math. Soc., to appear.

Département de mathématiques, Université du Québec À Montréal, P. O. Box 8888, Station A, Montréal, Québec, Canada H3C 3P8

Current address, X. Zhang: Department of Pure Mathematics, University of Waterloo, Waterloo, Ontario, Canada N2L 3G1

E-mail address: boyer@math.uqam.ca

E-mail address: zhang@math.uqam.ca 